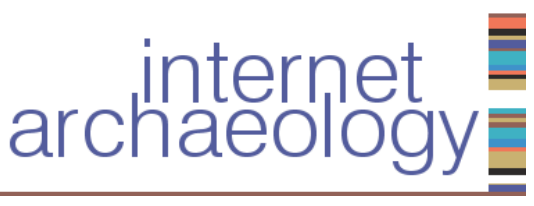

the premier e-journal for archaeology

\title{
Quaternary pollen analysis in the Iberian Peninsula: the value of negative results
}

\author{
J.S. Carrión ${ }^{1}$, S. Fernández로 , P. González-Sampériz², S.A.G. Leroy ${ }^{3}$, G.N. Bailey ${ }^{4}$, J.A. \\ López-Sáez $^{5}$, F. Burjachs ${ }^{6-7}$, G. Gil-Romera ${ }^{8}$, M. García-Antón ${ }^{9}$, M.J. Gil-García10, I. Parra ${ }^{11}$, \\ L. Santos ${ }^{12}$, P. López-García ${ }^{5}$, YII, E.I. ${ }^{6}$, M. Dupré ${ }^{13}$ \\ 1 Departamento de Biología Vegetal, Facultad de Biología, Universidad de Murcia, E-30100 Murcia, Spain \\ 2 Instituto Pirenaico de Ecología-CSIC, Avenida Montañana 1005, E-50059 Zaragoza, Spain \\ 3 Institute for the Environment, Brunel University, Uxbridge (West London), Middlesex UB8 3PH, United Kingdom \\ 4 Department of Archaeology, University of York, The King's Manor, York, Y01 7EP, United Kingdom \\ 5 Grupo de Investigación Arqueobiología, Instituto de Historia, CCHS, CSIC, Albasanz 26-28, 28037 Madrid, Spain \\ 6 Catalan Institution for Research and Advanced Studies (ICREA). Passeig Lluís Companys 23, E-08010 Barcelona, Spain \\ 7 Institut Català de Paleoecologia Humana i Evolució Social (IPHES). Àrea de Prehistòria, Universitat Rovira i Virgili, Plaça Imperial Tarraco 1, E-43005 Tarragona, Spain \\ 8 Institute of Geography and Earth Sciences, University of Wales, SY23 3DB Aberystwyth, United Kingdom \\ 9 Faculty of Sciences, Autonomous University of Madrid, E-28049 Cantoblanco, Madrid, Spain \\ 10 Department of Geology, University of Alcalá, E-28871 Madrid, Spain \\ 11 Observatorio Impactos Climáticos del Levante almeriense, OBAL LEAL, Garrucha, Spain \\ 12 Department of Earth Sciences, Faculty of Sciences, Campus da Zapateira s/n, University of Coruña, E-15071, A Coruña (Spain) \\ 13 Department of General Geography, Faculty of Geography and History, University of Valencia, Spain
}

(C) Internet Archaeology/Author(s) / URL: http://intarch.ac.uk/journal/issue25/5/toc.html / ISSN 1363-5387

\section{Summary}

Most unsuccessful palynological work is never published. As a consequence, pollen analysts waste time re-processing sterile sediments, and the available literature exhibits a uniformly positive record of success in pollen extraction. Here we report failures with Quaternary pollen analyses in the Iberian Peninsula; that is, case studies where it was not possible to extract palynomorphs for pollen counting. Both totally sterile and partially sterile sites are considered. Sites and perspectives for future studies are suggested. The majority of the failed studies are openair archaeological and palaeontological sites, caves and

* Corresponding author. J.S. Carrión

Email: carrion@um.es,

Phone and Fax: (34) 968364995 rockshelters, but there are prominent cases of success. Peat bogs have provided positive results, but only with sequences formed under continuous sedimentation processes in marshy environments. Lakes are often successful sites, but a multi-core strategy, following the facies change along a transect from the shore to the depositional centre, is recommended for saline lacustrine deposits, salt marshes and lagoons, especially when there is evidence of temporary desiccation. Cave and rockshelter infills should be considered case-by-case, and these sites definitely require a palyno-taphonomical approach to post-depositional processes. Indurated deposits are sometimes surprising in their high pollen concentration, but one must be prepared for sterility. Coprolites have been insufficiently exploited, and offer a great potential, especially those of Pleistocene Crocuta. This article shows that venturing into sediments assumed a priori to be 'difficult', like fluvial terraces, slope deposits, speleothems, cave travertines, and palaeosols, may nevertheless be successful. A 
summary is proposed of the various factors causing sterility, before, during and after sedimentation.

\section{Introduction}

Reporting failures is not a major concern in science. Research leading to failed or inconclusive results ends up most often unpublished, known only to those who did the work and quickly forgotten even by them. A notable exception is 'The Journal of Negative Results in Biomedicine'. In Quaternary palynology, while it is not unusual that the analyst is unable to extract pollen from sediments, the great bulk of unsuccessful work never sees the light of day and, as a consequence, the available palynological literature exhibits a misleadingly positive impression of success. In an interdisciplinary context, where the results of pollen analysis are often of interest to investigators who are not themselves palaeobotanists, such as archaeologists, palaeoclimatologists, palaeontologists and environmentalists, it is especially important that an understanding of the limitations of pollen analysis is widely disseminated. However, few nonpalaeoecologists seem to be sufficiently aware of something as obvious as the fact that not all sediments are suitable for pollen analysis (Bryant and Holloway 1983; Carrión et al. 1999a; López-Sáez et al. 2003; González-Sampériz 2004a). Often they may require or hope for palaeobotanical information from locations or deposits that are ill-suited to the preservation of pollen, or where the interpretation of the results is complicated by issues of differential preservation and taphonomy. Conversely, palaeobotanists may ignore deposits of potential interest or importance on the grounds that they are unlikely to produce adequate pollen, even though the results could be of great significance at a local scale or in relation to the problems investigated by other disciplines. Even palynologists who concentrate on the 'good' sites are at risk of wasting precious time and resources repeating pollen analyses of unproductive materials, since sites assumed a priori to be good sites may turn out to be sterile or partially so, just as sites assumed to be 'bad' may turn out to offer useful information. Applications for Quaternary palaeoecological research projects are often rejected on the grounds that they ignore earlier endeavours, but if the earlier endeavours are not published, everyone is at risk of wasting time and effort reinventing the same wheel and nothing is learned from earlier failures.

This article originates from an explicit commitment to report failures with pollen analyses for the Quaternary of the Iberian Peninsula. 'Failure' is understood here as the inability or impossibility of obtaining palynomorphs after following the usual extraction methods. A few cases include pollen spectra where the absolute number of pollen recovered from a sample was not sufficient for statistical treatment and interpretation. It is worth emphasising that we do not refer to unexpected or conflicting evidence or to results that are negative in the sense that they do not support hypotheses from designed experiments, nor do we mean those results unable to disprove a null hypothesis.
Our viewpoint is that, in spite of the difficulties of interpretation, failed pollen analyses will, sooner or later, be incorporated explicitly into the concepts of the discipline and its research procedures (e.g. Leroy 2008). In fact, a well-designed project should never produce a completely negative result, since there is always the opportunity to learn something. Learning about failures as well as positive results can be instrumental in providing the context for the development of new research strategies and so lead to a better return for public and private funding. Conversely, hearing only about the successes is equivalent to throwing away half of the information, and may give a misleading impression of the opportunities and limitations of pollen analysis. This issue of failure, or the production of unexpected results, is therefore of importance both to the non-specialist consumer of pollen results and the specialist palynologist, and our review is aimed at both types of reader.

\section{Methodological Considerations}

In addition to a review of the scanty available literature commenting on failures, this article principally uses the information obtained from a questionnaire submitted to Quaternary palynologists who, to our knowledge, have, at any time, been active in palynological work in the Iberian Peninsula. An e-mail list of 46 colleagues was built from directories of the APLE (Spanish-speakers Association of Palynologists), the AEQUA (Spanish Association of Quaternarists), the INQUA (International Union for Quaternary Research), and the IFPS (International Federation of Palynological Societies). We also made telephone calls to people who had been leading projects and initiatives related to Iberian palaeoecology. All the collaborators are named as authors of this article. Eight declared not to have found problems with their own analyses, in these cases exclusively conducted with material from peat bogs and lacustrine sediments. The remaining 23 individuals (50\% of the list) to whom the questionnaire was sent did not reply.

Why so many failed to participate is perhaps an interesting matter for sociological research that is outside the scope of this article. Possible causes are: lack of records of failed pollen work; a poor tradition of collaborative research; bad experiences with former database initiatives; perhaps even doubts about the need for this work. Some palynologists may also now be retired or deceased. In any case, it seems logical to consider that the number of sites listed here (221) is surely less than the total. In addition, even if there was a regional distribution among the non-answers, and taking into account that several areas of Iberia, like the humid north-west, have been more intensively explored and studied than others (Carrión et al. 2000a; 2008), this article cannot deal with possible geographic trends in sediment sterility. This is unfortunate, because the Iberian Peninsula contains an important physiographical heterogeneity (Vera 2004). Therefore with a more complete dataset, several tendencies might have become detectable. 
Table 1. Case studies with total (all samples processed) versus partial sterility in pollen analysis of Iberian sites.

\begin{tabular}{|l|l|l|l|l|l|l|}
\hline \multirow{2}{*}{ Site Type } & \multicolumn{2}{|c|}{ Number } & \multicolumn{2}{c|}{$\%$} & \multirow{2}{*}{ Total cases } \\
\cline { 2 - 6 } & Partial & Total & Partial & Total & \\
\hline Peat Bogs & 10 & 1 & 90,91 & 9,09 & 11 \\
\hline Non-saline lakes/palaeo-lakes & 16 & 9 & 64,00 & 36,00 & 25 \\
\hline Saline lacustrine systems & 19 & 3 & 86,36 & 13,64 & 22 \\
\hline Caves & 32 & 19 & 62,75 & 37,25 & 51 \\
\hline Rockshelters & 12 & 15 & 44,44 & 55,56 & 27 \\
\hline Open-air archaeological & 37 & 26 & 58,73 & 41,27 & 63 \\
\hline Open-air palaeontological & 2 & 4 & 33,33 & 66,67 & 6 \\
\hline Travertines & 0 & 2 & 0,00 & 100,00 & 2 \\
\hline Palaeosoils & 2 & 1 & 66,67 & 33,33 & 3 \\
\hline Fluvial terraces & 2 & 0 & 100,00 & 0,00 & 2 \\
\hline Slope deposits & 2 & 3 & 40,00 & 60,00 & 5 \\
\hline Moraine deposits & 0 & 1 & 0,00 & 100,00 & 1 \\
\hline Coprolites & 6 & 8 & 42,86 & 57,14 & 14 \\
\hline \hline
\end{tabular}

The methods of sampling and laboratory analysis declared by contributors are the usual ones. Thus, most drilling in lacustrine and peaty sediments was done using Russian, Hiller, piston and window corers and rotary drilling (Birks 1986; Leroy 1990). Only rarely were open sections sampled in accessible peat bogs (Carrión and van Geel 1999). Cave sediment sampling from stratigraphical sections followed Girard (1975); Burjachs et al. (2003), or similar (Dupré 1988). Coprolites were cut open and material from the centre was scraped out to minimise contamination from external surfaces (Carrión et al. 2001a). Sometimes, the totality of the coprolites was treated after cleaning the surface with distilled water (González-Sampériz et al. 2003b). Independent of the materials, laboratory treatment was performed following the classical $\mathrm{HCl}, \mathrm{HF}$ and KOH method (e.g. Girard and Renault-Miskovsky 1969; Faegri and Iversen 1975; Moore et al. 1991; Bennett and Willis 2001). Mineral separation with heavy liquids (Goeury and de Beaulieu 1979; Dricot and Leroy 1989; Nakagawa et al. 1998) was common not only for minerogenic sediments, but also in organic layers of salt marshes, deltas, lagoonal sediments and lacustrine ones. In other cases, sieving was done at 10 microns and also at a coarser mesh (larger than the largest pollen grain). So, even with presumably pollen-rich sediments, Iberian palynologists tend to use complex concentration methods. Could this tradition be related to a long experience of difficulties with extracting pollen and to the diversity of the sediment when available?
Although it is generally not possible to know whether the best analytical procedure was correctly applied, to blame pollen-analysts for failures of pollen extraction seems a little unrealistic. Certainly, macerating larger samples, using sodium pyrophosphate for clays, and gravity separation to enhance pollen concentration, among other protocols, can solve some problems of concentration (Horowitz 1992). But experimental work (Birks and Birks 1980; Havinga 1984; Tipping 1987; Jones et al. 2007) suggests that, in a number of cases, the absence of pollen can be attributable to the nature of the depositional environment. Our primary goal is informative, that is, once problems with a site are known, the pollen analyst should be free to repeat the analysis or avoid further trials.

\section{Incidence of Failure Discussed by Type of Depositional Site}

In this section, sites are presented by type of archive. In the next section, mechanisms are proposed that may account for the sterility.

Compiled failures comprise 221 sites, which are here organised by depositional/sedimentary types, and information is given about their location, magnitude of the sterility (all samples versus only particular levels/samples), age or presumed chronology, and the name and affiliation of the pollen-analyst/s (Tables 1-7, Fig. 2 and Fig. 2). Open-air archaeological sites 


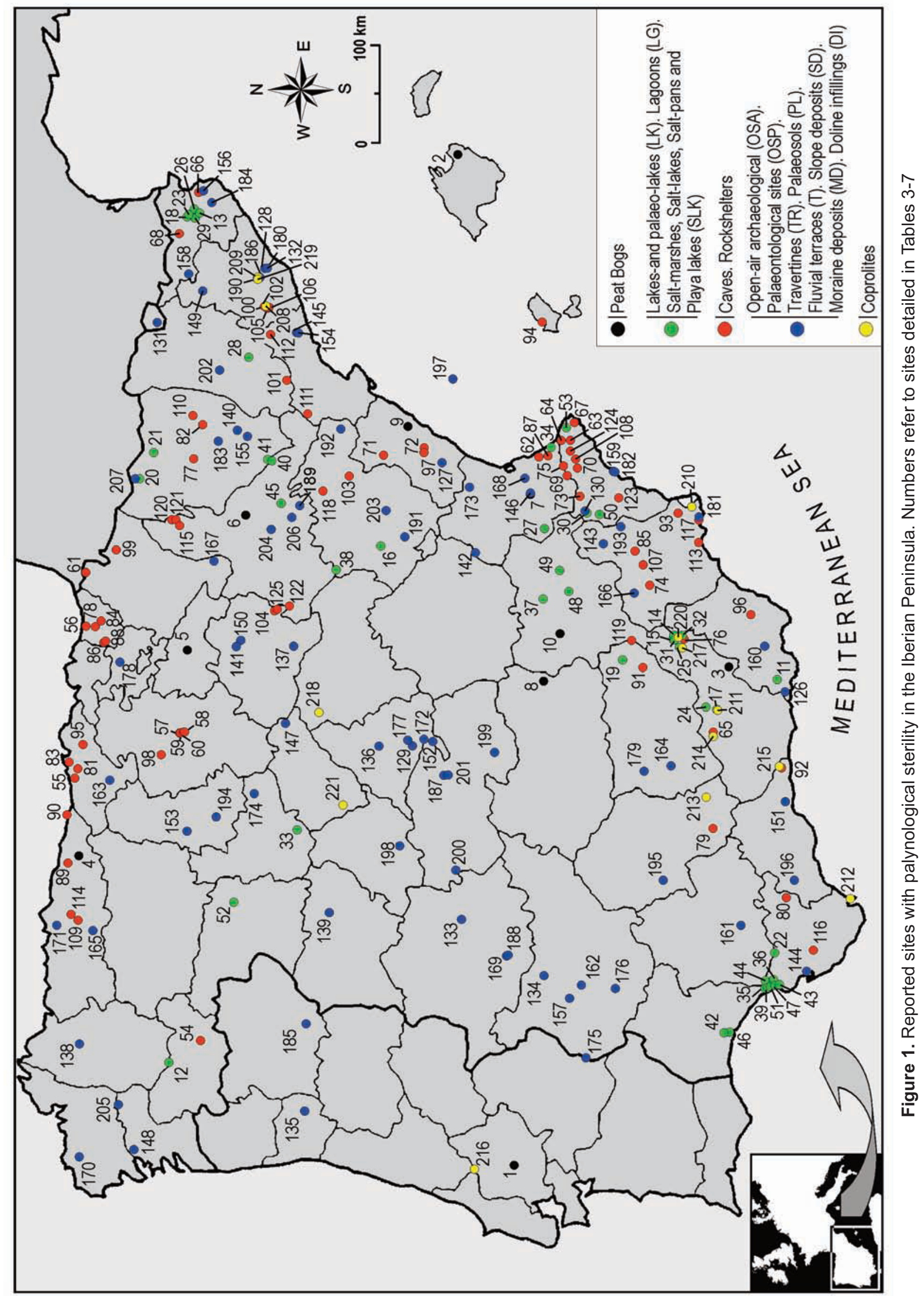




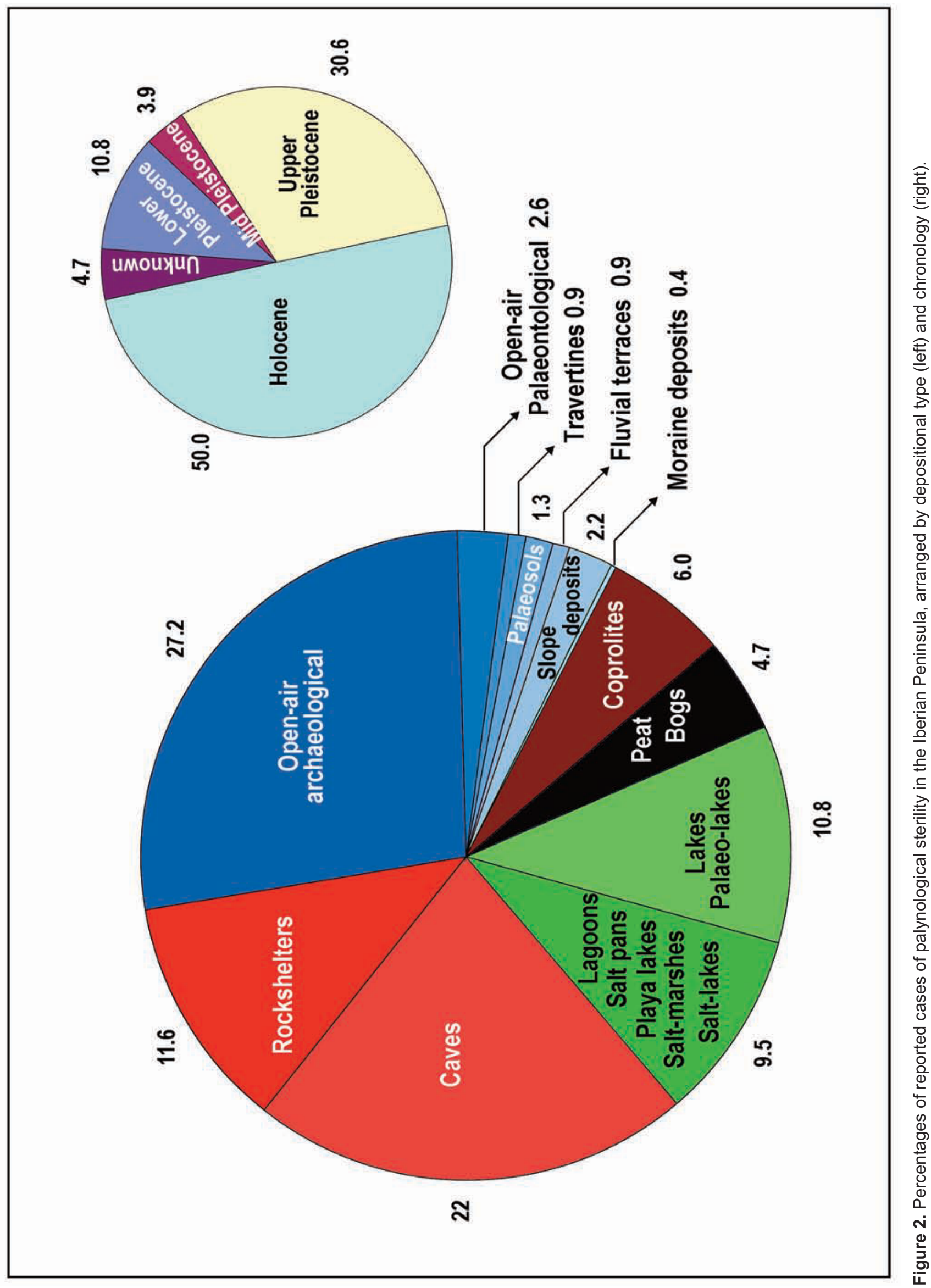


Table 2. Abbreviations for the analysts of the pollen-sterile sites (Tables 3-7).

\begin{tabular}{|c|c|c|}
\hline Analyst & Research centre & Abbreviation \\
\hline AIRA, M.J. & University of Santiago & MJA \\
\hline ALLUÉ, E. & $\begin{array}{l}\text { Catalan Institute of Human Palaeoecology and Social } \\
\text { Evolution (IPHES), Tarragona }\end{array}$ & EA \\
\hline BOYER-KLEIN, A. & Musée de l'Homme, Paris & BK \\
\hline BURJACHS, F. & $\begin{array}{l}\text { ICREA at Catalan Institute of Human Palaeoecology } \\
\text { and Social Evolution (IPHES). Tarragona }\end{array}$ & FB \\
\hline CARRIÓN, J.S. & University of Murcia & JC \\
\hline DAVIS, $\mathrm{B}$. & University of Neweastle & $\mathrm{BD}$ \\
\hline DUPRÉ, M. & University of Valencia & MD \\
\hline ESTÉBAN, A. & Ajuntament d'Esterri de Cardós & $\mathrm{AE}$ \\
\hline EXPÓSITO, I. & $\begin{array}{l}\text { Catalan Institute of Human Palacoecology and Social } \\
\text { Evolution (IPHES), Tarragona }\end{array}$ & IE \\
\hline FERNÁNDEZ, S, & University of Murcia & SF \\
\hline $\begin{array}{l}\text { GARCLA-ANTÓN, } \\
\text { M. }\end{array}$ & Autonomous University of Madrid & MGA \\
\hline GEURTS, M. & University of Ottawa, Canada & MGU \\
\hline GIL-GARCIA, M.J. & University of Alcalá, Madrid & MGG \\
\hline GIL-ROMERA, G. & University of Wales, Aberystwyth & GR \\
\hline $\begin{array}{l}\text { GONZÁLEZ. } \\
\text { SAMPÉRIZ, P. }\end{array}$ & Instituto Pirenaico de Ecologia, Zaragoza & PGS \\
\hline IRIARTE, M.J. & University of Pais Vasco, Bilbao & MЛ \\
\hline JANSSEN, C. & University of Utrecht & CJ \\
\hline $\begin{array}{l}\text { LEROI-GOURHAN, } \\
\text { A. }\end{array}$ & Musée de l'Homme, Paris & ALG \\
\hline LEROY, S.A.G. & Brunel University, London & SL \\
\hline LÓPEZ-GARCI A.P. & Institute of History, Madrid & PLG \\
\hline LÓPEZ-SÁEZ, J.A. & Institute of History, Madrid & JS \\
\hline MARISCAL, B. & University Complutense, Madrid & BM \\
\hline $\begin{array}{l}\text { MARTIN-ARROYO, } \\
\text { T. }\end{array}$ & University of Alcalä, Madrid & TMA \\
\hline $\begin{array}{l}\text { MENÉNDEZ-AMOR, } \\
\text { J. }\end{array}$ & University Complutense, Madrid & JMA \\
\hline MUNUERA, M. & Polytechnie University of Cartagena, Murcia & MM \\
\hline PARRA, I. & SINKLIM, Almeria & IP \\
\hline PÉREZ-OBIOL, $\mathrm{R}$. & Autonomous University of Barcelona & RPO \\
\hline RAMIL-REGO.P. & University of Santiago & PRR \\
\hline $\begin{array}{l}\text { RENAULT- } \\
\text { MISKOVSKY, J. }\end{array}$ & Musée de l'Homme, Paris & JRM \\
\hline RUIZ-ZAPATA, M.B. & University of Alealä, Madrid & BRZ \\
\hline $\begin{array}{l}\text { SANCHEZ-GOÑI, } \\
\text { M.F. }\end{array}$ & University of Bordeaux I & MFSG \\
\hline SANCHIS, A.K. & University of Valencia & AKS \\
\hline SANTOS, L. & University of Coruña & LS \\
\hline STEVENSON, A.C, & University of Neweastle, UK & ACS \\
\hline SUC, J.P. & University of Montpellier & SuC \\
\hline $\begin{array}{l}\text { VAN DER KNAAP. } \\
\text { W.O. }\end{array}$ & University of Bern & VKN \\
\hline VAN GEEL, B. & University of Amsterdam & VG \\
\hline VAN LEEUWEN, $J$. & University of Bern & VLW \\
\hline VAN MOURIK, J.M. & University of Amsterdam & VMO \\
\hline YÁÑEZ,C. & University Pablo de Olavide, Sevilla & $\mathrm{CY}$ \\
\hline VOLMAN, K.C. & University of Cantabria, Santander & KV \\
\hline YLL, R. & $\begin{array}{l}\text { Catalan Institute of Human Palaeoecology and Social } \\
\text { Evolution (IPHES), Tarragona }\end{array}$ & RY \\
\hline
\end{tabular}

$(27.2 \%)$, caves $(22 \%)$ and rockshelters $(11.6 \%)$ represent a majority of the failed case studies (Fig. 2). The proportion of nonsaline (10.8\%) and saline lacustrine systems (including lagoons, salt pans, playa lakes, salt marshes, and salt lakes) $(9.5 \%)$ is higher than peat bogs $(4.7 \%)$. Coprolites $(6 \%)$ were sterile either individually, or collectively by site (Table 7). Our files also include a few cases with open-air palaeontological sites (2.6\%), fluvial terraces $(0.9 \%)$, slope deposits $(2.2 \%)$, moraine deposits $(0.4 \%)$, palaeosols $(1.3 \%)$, and exposed travertines $(0.9 \%)$ (Fig. 2, Table 6). Chronologically, $50 \%$ of the failed sites are
Holocene, 30.6\% Upper Pleistocene, 10.8\% Lower Pleistocene, and $3.9 \%$ Middle Pleistocene. These percentages are likely to be related to the availability of deposits by age. Sites of unknown age average $4.7 \%$ of the reported total (Fig. 2).

Complete sterility notoriously affects open-air palaeontological sites $(67 \%)$, and is also relatively high in coprolites (57\%), slope deposits $(60 \%)$, rockshelters $(55 \%)$, open-air archaeological sites (41\%), caves (37\%), and non-saline lakes $(36 \%)$, mostly in cases of palaeolakes (Table 1). Complete sterility is only $9 \%$ in peat bogs (Table 1$)$. The few samples of travertines and moraine deposits reported a total absence of pollen. Complete sterility averaged $19 \%$ of conventional (lacustrine and peaty) pollen sites. In contrast, it averaged 50\% in archaeological sites, including caves, rockshelters, and openair archaeological or palaeontological excavation sites. Thus, the potential of success at re-studying failed sites is clearly higher in the open-air group.

\subsection{Peat bogs}

Peat is a classic model for Quaternary pollen analysis (Birks and Birks 1980). However, peat bogs are rare in the Mediterranean region of Iberia, and not particularly numerous in its wetter, Eurosiberian, region (Turner and Hannon 1988; Peñalba 1994; Ramil-Rego et al. 1998; González-Sampériz et al. 2005). Long pollen sequences (e.g. more than $10 \mathrm{~m}$ ) obtained from Iberian peat deposits like Padul in Andalucía (Florschütz et al. 1971; Pons and Reille 1988), Quintanar de la Sierra in the northern Meseta (Peñalba et al. 1997), El Portalet in the Pyrenees (González-Sampériz et al. 2006), and Area Longa in Galicia (Gómez-Orellana et al. 2007) are exceptional.

Given that peats may occur in different wetlands (e.g. peat bogs, fens, swamps, marshes) resulting from a complexity of geomorphological and sedimentary situations, sediments obtained after coring peat deposits are not always peaty throughout. Inorganic layers within mires are problematic for pollen analysis (Moore 1986; Barber and Charman 2003). Several of the records considered here show pollen in sediments formed under continuous sedimentation processes in marshy and shallow lacustrine environments. However, they also show palynologically sterile levels in fluvial and marine depositional environments (Table 3). This could be the situation with the Torreblanca peat bog (Dupré et al. 1994), but is demonstrated most clearly with Navarrés (Valencia), a tectonic, flat-bottom valley from which only the uppermost $250 \mathrm{~cm}$ of a $25 \mathrm{~m}$ core were polliniferous (Carrión and Dupré 1996; Carrión and van Geel 1999) (Table 2). In particular, this is the section corresponding to the accumulation of peat in a waterlogged context (Dupré et al. 1998a). The rest of the Quaternary sequence, starting at $c .178,000$ years BP, is dominated by high-energy fluvial facies. Samples between 166 and $145 \mathrm{~cm}$ depth were also palynologically sterile, a hiatus corresponding with the Last Glacial Maximum, during which conditions were not favourable for biotic preservation over large areas of the basin (Carrión and van Geel 1999). A former study including two cores, taken 
Table 3. Reported cases of palynological sterility in peat bog sites of the Iberian Peninsula.

\begin{tabular}{|c|c|c|c|c|c|c|c|}
\hline$\frac{\text { Site }}{\text { Number }}$ & Site (province) & $\begin{array}{c}\text { Coordinates Altitude } \\
\mathrm{m} \text { asl }\end{array}$ & Age & Sterility & Reference & Analvst & $\begin{array}{l}\text { Lab } \\
\text { Year }\end{array}$ \\
\hline 1 & Alpiarça (Portugal) & $\begin{array}{l}39^{\circ} 15^{\prime} 36^{\prime \prime} \mathrm{N} \\
8^{\circ} 35^{\prime} 04^{\prime \prime} \mathrm{W} \\
20\end{array}$ & $5000-2000 \mathrm{BP}$ & Partial & $\begin{array}{l}\text { van Leeuwaarden \& Janssen } \\
1985\end{array}$ & $\begin{array}{l}\text { VLW- } \\
\text { CJ }\end{array}$ & - \\
\hline 2 & Artá (Mallorea) & $\begin{array}{l}39^{\circ} 41^{\prime} 23^{\prime \prime} \mathrm{N} \\
3^{\circ} 19^{\prime \prime} 4^{\prime \prime} \mathrm{E} \\
180\end{array}$ & Holocene & Total & Roure et al. 2000 & $\begin{array}{l}\text { RY- } \\
\text { RPO }\end{array}$ & 1998 \\
\hline 3 & Baza (Granada) & $\begin{array}{l}37^{\circ} 14^{\prime} 35^{\prime \prime} \mathrm{N} \\
2^{\circ} 42^{\prime} 23^{\prime \prime} \mathrm{W} \\
1900\end{array}$ & Holocene & Partial & Carrión et al. $\underline{2007 \mathrm{~b}}$ & JC & 2003 \\
\hline 4 & Comella (Asturias) & $\begin{array}{l}43^{\circ} 16^{\prime} 55^{\prime \prime} \mathrm{N} \\
4^{\circ} 59^{\prime} 15^{\prime \prime} \mathrm{W} \\
850\end{array}$ & Holocene & Partial & Ruiz-Zapata et al. $\underline{2000}$ & BRZ & - \\
\hline 5 & $\begin{array}{l}\text { Los Monjes (La } \\
\text { Rioja) }\end{array}$ & $\begin{array}{l}42^{\circ} 14^{\prime} 28^{\prime \prime} \mathrm{N} \\
2^{\circ} 32^{\prime} 51^{\prime \prime} \mathrm{W} \\
1450\end{array}$ & Last 2 ka & Partial & Gil-Garcia et al. 1995 & MGG & 1993 \\
\hline 6 & $\begin{array}{l}\text { Mozarrifar I } \\
\text { (Zaragoza) }\end{array}$ & $\begin{array}{l}41^{\circ} 44^{\prime} 35^{\prime \prime} \mathrm{N} \\
0^{\circ} 51^{\prime} 50^{\prime \prime} \mathrm{W} \\
220^{\prime}\end{array}$ & Pleistocene & Partial & González-Sampériz et al. $\underline{2005}$ & PGS & 2002 \\
\hline \multirow[t]{2}{*}{7} & \multirow[t]{2}{*}{ Navarrés (Valencia) } & \multirow{2}{*}{$\begin{array}{l}39^{\circ} 6^{\prime} 5^{\prime \prime} \mathrm{N} \\
0^{\circ} 41^{\prime} 36^{\prime \prime} \mathrm{W} \\
225\end{array}$} & \multirow[t]{2}{*}{$173-3 \mathrm{ka}$} & \multirow[t]{2}{*}{ Partial } & $\begin{array}{l}\text { Menéndez-Amor \& Florschtitz } \\
1961\end{array}$ & JMA & \multirow[t]{2}{*}{$\begin{array}{l}1960- \\
1996\end{array}$} \\
\hline & & & & & Carriỏn \& Duprế 1996 & JC-VG & \\
\hline 8 & $\begin{array}{l}\text { Ruidera (Ciudad } \\
\text { Real) }\end{array}$ & $\begin{array}{l}38^{\circ} 58^{\prime} 31^{\prime \prime N} \\
2^{\circ} 53^{\circ} 03^{\prime \prime} \mathrm{W} \\
800\end{array}$ & Holocene & Partial & Julià et al. 1994a & FB & 1992 \\
\hline 9 & $\frac{\text { Torreblanca }}{\text { (Castellón) }}$ & $\begin{array}{l}40^{\circ} 11^{\prime} 50^{\prime \prime} \mathrm{N} \\
0^{\circ} 12^{\prime} 39^{\prime \prime} \mathrm{E} \\
1\end{array}$ & $6040-<2600 \mathrm{BP}$ & Partial & Dupré et al. 1994 & MD & 1991 \\
\hline
\end{tabular}

$5 \mathrm{~m}$ apart in another part of the basin, had reported pollen only in the uppermost 180cm (Carrión and Dupré 1996), and the prevailing minerogenic sediments of a Neolithic settlement site in the vicinity were poor in pollen, and totally sterile in sandy sediments (Dupré et al. 1985). A similar pattern of pollen occurrence is described in a pioneer study by Menéndez-Amor and Florschütz (1961).

In the Arroyo de los Monjes (La Rioja), a 90cm-depth peaty sand core was fully sterile (Table 3 ). The 8000-year pollen record from Comella peat bog (Asturias, near Covadonga lakes) was produced from the uppermost $5.7 \mathrm{~m}$ of peaty sediment, while the underlying detritic, sandy silt section did not contain any pollen (Ruíz-Zapata et al. 2002). In the Alpiarça peat bog (Portugal), clayey levels were sterile (van Leeuwaarden and Janssen 1985). In several limnic deposits from Galicia, peats occurring between thick detritic, sterile layers were the only sediments successfully analysed (Ramil-Rego and GómezOrellana 1996).

The case of Cañada del Gitano in the Sierra de Baza (Granada) could also be related to the abundance of detritic materials in parts of the peat bog. Two sediment cores were collected from the head of this deposit, and coring stopped at 417 and $378 \mathrm{~cm}$ on reaching bedrock (Carrión et al. 2007b). While the $378 \mathrm{~cm}$-deep core was fully polliniferous, the longer $417 \mathrm{~cm}$ deep core was discarded because of its poor pollen content, with total sterility in several layers. The polliniferous core was mainly peat and silty peat, while the sterile one consisted of clastic silt.

Alteration of the original sedimentary structure may lead to sterility in peats. This is perhaps what happened to one of the two cores from Villaverde (Albacete), a tufaceous peat deposit overlying a calcreted conglomerate bedrock (Table 3). A sediment core of $550 \mathrm{~cm}$ depth obtained from the eastern part of the fan was useful for pollen analysis (Carrión et al. 2001b). However, another one of $c .490 \mathrm{~cm}$ depth obtained in the northern area was almost completely sterile, showing signs of corrosion in the few pollen grains and spores observed. In this case, the sedimentary context, a detrital marl interbedded with peats and sapropels, was identical for both cores (Carrión et al. 2001b). An earlier study by Taylor et al. (1998) based on a $600 \mathrm{~cm}$-core from the western part of the fan had already pointed to the abundance of inorganic matter and the investigators complained about poor pollen preservation. After reviewing unpublished reports provided by the landowner, we observed that, over the preceding years, the northern and western parts of the basin had been subject to trench excavation for a peat exploitation project requiring drainage.

\subsection{Lakes, salt marshes, salt pans, playa lakes, and lagoons}

With few exceptions (Dupré 1988; Dupré et al. 1996; Davis 1994; Leroy 1990; 1997; 2008; Pérez-Obiol and Julià 1994; van der Knaap and van Leeuwen 1994; 1995), lake sediments from the Iberian Peninsula have been extensively explored for pollen only during the last decade (Burjachs et al. 1997; Carrión et al. 2001c; 2004b; Carrión 2002a; Muñoz-Sobrino et al. 2004; Valero-Garcés et al. 2006a; González-Sampériz et al. 2005; 2008; Morellón et al. 2008; Moreno et al. in press). In general, karstic lakes with permanent freshwater, and riverine wetlands and floodplains, are valuable for pollen analysis. But there are exceptions (Table 4). Pollen was absent in the less organic sediments of San Benito (Dupré et al. 1996) and Beco- 
Table 4. Reported cases of palynological sterility in lacustrine sites.

\begin{tabular}{|c|c|c|c|c|c|c|c|c|}
\hline $\begin{array}{c}\text { Site } \\
\text { Number }\end{array}$ & Site (province) & $\begin{array}{l}\text { Coordinates } \\
\text { Altitude } \mathrm{m} \text { asl }\end{array}$ & $\begin{array}{l}\text { Site } \\
\text { type }\end{array}$ & Age & Sterility & Reference & Analvst & $\begin{array}{l}\text { Lab } \\
\text { year }\end{array}$ \\
\hline 11 & $\begin{array}{l}\text { Balsa del Sabinar } \\
\text { (Almeria) }\end{array}$ & $\begin{array}{l}36^{\circ} 53^{\prime} 03^{\prime \prime} \mathrm{N} 2^{\circ} 51^{\prime} \\
32^{\prime \prime} \mathrm{W} 1827^{\prime}\end{array}$ & LK & - & Partial & Carrión et al. 2003a & MD-JC & 2000 \\
\hline 12 & Becorreiras (Ourense) & $\begin{array}{l}42^{\circ} 15^{\prime} 18^{\prime \prime} \mathrm{N}^{\circ} 7^{\circ} 22^{\prime} \\
46^{\prime \prime} \mathrm{W} 1320\end{array}$ & LK & $8336-7992$ cal BP & Partial & Santos $\underline{2004}$ & LS & 1991 \\
\hline 13 & $\begin{array}{l}\text { Bòbila Ordis, Lake I } \\
\text { (Girona) }\end{array}$ & $\begin{array}{l}42^{\circ} 08^{\prime} 21^{\prime \prime} \mathrm{N} 2^{\circ} 44^{\prime} \\
5^{\prime \prime} \mathrm{E} 210\end{array}$ & LK & Lower Pleistocene & Partial & $\begin{array}{l}\text { Lovlie \& Leroy 1995; } \\
\text { Leroy } 2008\end{array}$ & SL & $\begin{array}{l}1989- \\
2004\end{array}$ \\
\hline 14 & Concjos (Granada) & $\begin{array}{l}37^{\circ} 43^{\prime} 18^{\prime \prime} \mathrm{N} 2^{\circ} 27^{\prime} \\
1^{\prime \prime} \mathrm{W} 991\end{array}$ & $\begin{array}{l}\text { LK } \\
\text { OSP }\end{array}$ & Lower Pleistocene & Total & Gibert et al. 1988 & SL & $\begin{array}{l}\text { Late } \\
1980 \text { s }\end{array}$ \\
\hline 15 & $\begin{array}{l}\text { Champiñones } \\
\text { (Granada) }\end{array}$ & $\begin{array}{l}37^{\circ} 43^{\prime} 58^{\prime \prime} \mathrm{N} 2^{\circ} 23^{\prime} \\
16^{\prime \prime} \mathrm{W} 970\end{array}$ & $\begin{array}{l}\text { LK } \\
\text { OSP }\end{array}$ & Lower Pleistocene & Total & Gibert et al. 1988 & SL & $\begin{array}{l}\text { Late } \\
1980 \text { s }\end{array}$ \\
\hline 16 & \begin{tabular}{|l|} 
El Cañizar, \\
Villarquemado \\
(Teruel)
\end{tabular} & $\begin{array}{l}40^{\circ} 30^{\prime} 8^{\prime \prime} \mathrm{N}^{\circ} 17^{\prime} \\
7^{\prime \prime} \text { W } 987\end{array}$ & LK & $130-4$ ka & Partial & $\begin{array}{l}\text { Valero-Garcés et al. } \\
2006 \mathrm{~b}\end{array}$ & JC-SF & $2005-7$ \\
\hline 17 & Fonelas (Granada) & $\begin{array}{l}37^{\circ} 24^{\prime} 45^{\prime \prime} \mathrm{N} 3^{\circ} 12^{\prime} \\
10^{\prime \prime} \mathrm{W} 800\end{array}$ & $\begin{array}{l}\text { LK } \\
\text { OSP }\end{array}$ & Plio-Pleistocene & Total & Arribas et al, $2004 \mathrm{~b}$ & JC & $2001-5$ \\
\hline \multirow{3}{*}{18} & \multirow{3}{*}{$\begin{array}{l}\text { Incarcal-Crespia } \\
\text { (Girona) }\end{array}$} & \multirow{3}{*}{$\begin{array}{l}42^{\circ} 11^{\prime} 13^{\prime \prime} \mathrm{N} 2^{\circ} 47^{\prime} \\
59^{\prime \prime} \mathrm{E} 130\end{array}$} & \multirow{3}{*}{$\begin{array}{l}\mathrm{LK} \\
\text { OSP }\end{array}$} & \multirow{3}{*}{ Lower Pleistocene } & \multirow{3}{*}{ Partial } & Geurts $1977: 1979$ & MGU & \multirow{3}{*}{$\begin{array}{l}1980- \\
90\end{array}$} \\
\hline & & & & & & Suc 1980 & SUC-FB & \\
\hline & & & & & & Leroy 1990 & SL & \\
\hline 19 & \begin{tabular}{|l} 
Laguna de Orcera \\
(Jaén)
\end{tabular} & $\begin{array}{l}38^{\circ} 19^{\prime} 2^{\prime \prime} \mathrm{N} 2^{\circ} 39^{\prime} \\
1^{\prime \prime} \mathrm{W} 900\end{array}$ & LK & Holocene & Total & - & JC & 2000 \\
\hline 20 & Lana Mayor (Huesca) & $\begin{array}{l}42^{\circ} 42^{\prime} 51^{\prime \prime} \mathrm{N}^{\circ} 18^{\prime} \\
59^{\prime \prime} \mathrm{W} 1600\end{array}$ & LK & - & Total & $\begin{array}{l}\text { González-Sampériz } \\
2001\end{array}$ & PGS & 1999 \\
\hline 21 & $\begin{array}{l}\text { Linás de Broto } \\
\text { (Huesea) }\end{array}$ & $\begin{array}{l}42^{\circ} 36^{\prime} 19^{\prime \prime} \mathrm{N}^{\circ} 7^{\prime} \\
21^{\prime \prime} \mathrm{W} 1250\end{array}$ & LK & $>33 \mathrm{ka}$ & Partial & $\begin{array}{l}\text { González-Sampériz et } \\
a l .2005\end{array}$ & PGS & 1999 \\
\hline 22 & Los Tollos (Cádiz) & $\begin{array}{l}36^{\circ} 50^{\prime} 43^{\prime \prime} \mathrm{N} 6^{\circ} 1^{\prime} \\
3^{\prime \prime} \text { W } 54\end{array}$ & LK & Pleistocene & Partial & - & SF & 2007 \\
\hline \multirow{2}{*}{23} & \multirow{2}{*}{ Mas Miquel (Girona) } & \multirow{2}{*}{$\begin{array}{l}42^{\circ} 10^{\prime} 52^{\prime \prime} \mathrm{N} 2^{\circ} 48^{\circ} \\
27^{\prime \prime} \mathrm{E} 100\end{array}$} & \multirow{2}{*}{ LK } & \multirow{2}{*}{ Lower Pleistocene } & \multirow{2}{*}{ Partial } & Geurts $\underline{1977}$ & MGU & \multirow{2}{*}{$\begin{array}{l}1977 . \\
90\end{array}$} \\
\hline & & & & & & Leroy 1990 & SL. & \\
\hline 24 & Mencal (Granada) & $\begin{array}{l}37^{\circ} 29^{\prime} 38^{\prime \prime} \mathrm{N} 3^{\circ} 9^{\prime} \\
49^{\prime \prime} \mathrm{W} 920\end{array}$ & $\begin{array}{l}\text { LK } \\
\text { OSP }\end{array}$ & Plio-Pleistocene & Total & Arribas et al. 2004b & $\mathrm{SF}$ & 2007 \\
\hline 25 & Orce (Granada) & $\begin{array}{l}37^{\circ} 43^{\prime} 17^{\prime \prime} \mathrm{N} 2^{\circ} 28^{\prime} \\
45^{\prime \prime} \mathrm{W} 940\end{array}$ & $\begin{array}{l}\text { LK } \\
\text { OSP }\end{array}$ & Lower Pleistocene & Total & $\begin{array}{l}\text { Gibert ef al. } 1988: \\
\text { Agusti \& Julia } 1990\end{array}$ & FB-SL-IP & - \\
\hline 26 & $\begin{array}{l}\text { Pla de l'Estany } \\
\text { (Girona) }\end{array}$ & $\begin{array}{l}41^{\circ} 52^{\prime} 6^{\prime \prime} \mathrm{N} 2^{\circ} 6^{\prime} \\
48^{\prime \prime} \mathrm{E} 870\end{array}$ & LK & $\begin{array}{l}\text { Upper Pleistocene- } \\
\text { Holocene }\end{array}$ & Partial & $\begin{array}{l}\text { Pérez-Obiol 1988; } \\
\text { Burjachs } 1994\end{array}$ & RPO-FB & 1986 \\
\hline 27 & San Benito (Valencia) & $\begin{array}{l}38^{\circ} 56^{\circ} 30^{\prime \prime} \mathrm{N}^{\circ} 6^{\prime} \\
30^{\prime \prime} \mathrm{W} 671\end{array}$ & LK & $>41000-1410 \mathrm{BP}$ & Partial & Dupré et al. 1996 & MD & 1995 \\
\hline 28 & Tomabous (Lleida) & 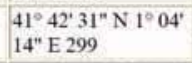 & LK & Holocene & Partial & Yil et al. $2008 \mathrm{c}$ & $\begin{array}{l}\text { RY-FB- } \\
\text { IE }\end{array}$ & 2007 \\
\hline 29 & Tres Pins (Girona) & $\begin{array}{l}42^{\circ} 08^{\circ} 45^{\prime \prime} \mathrm{N} 2^{\circ} 43^{\prime} \\
5^{\prime \prime} \mathrm{E} 210\end{array}$ & LK & $\begin{array}{l}\text { Late Pliocene- } \\
\text { Lower Pleistocene }\end{array}$ & Partial & Leroy 1997 & SL & 1988 \\
\hline 30 & Villena (Alicante) & $\begin{array}{l}38^{\circ} 37^{\prime} 11^{\prime \prime} \mathrm{N} 0^{\circ} 55^{\prime} \\
49^{\prime \prime} \mathrm{W} 502\end{array}$ & LK & $\begin{array}{l}\text { Upper Pleistocene- } \\
\text { Holocene }\end{array}$ & Partial & YIl et al. 2003 & $\begin{array}{l}\text { EY-RPO- } \\
\text { MD }\end{array}$ & 2001 \\
\hline 31 & Yeseras (Granada) & $\begin{array}{l}37^{\circ} 47^{\prime} 59^{\prime \prime} \mathrm{N} 2^{\circ} 27^{\prime} \\
48^{\prime \prime} \mathrm{W} 1065\end{array}$ & $\begin{array}{l}\text { LK } \\
\text { OSP }\end{array}$ & Lower Pleistocene & Total & Agusti \& Juliá 1990 & SL & $\begin{array}{l}\text { Late } \\
1980 \text { s }\end{array}$ \\
\hline 32 & Zagales (Granada) & $\begin{array}{l}37^{\circ} 44^{\prime} 19^{\prime \prime} \mathrm{N} 2^{\circ} 26^{\prime} \\
5^{\prime \prime} \text { W } 970\end{array}$ & $\begin{array}{l}\text { LK } \\
\text { OSP }\end{array}$ & Lower Pleistocene & Total & Gibent et al. 1988 & SL & $\begin{array}{l}\text { Late } \\
1980 \mathrm{~s}\end{array}$ \\
\hline 33 & $\begin{array}{l}\text { Almenara de Adaja } \\
\text { (Valladolid) }\end{array}$ & $\begin{array}{l}41^{\circ} 12^{\prime} 48^{\prime \prime} \mathrm{N} 4^{\circ} 40^{\prime} \\
40^{\prime \prime} \mathrm{W} 780\end{array}$ & LG & $>2675 \mathrm{BP}$ & Partial & Delibes \& Moure 1973 & JLS & 2003 \\
\hline 34 & Pege (Valencia) & $\begin{array}{l}38^{\circ} 51^{\prime} 52^{\prime \prime} \mathrm{N} 0^{\circ} 3^{\prime} \\
11^{\prime \prime} \mathrm{W} 4\end{array}$ & LG & $8300-7800 \mathrm{BP}$ & Partial & Dupré et al. $1998 \mathrm{~b}$ & MD & 1986 \\
\hline 37 & $\frac{\text { El Acequiôn }}{\text { (Albacete) }}$ & $\begin{array}{l}38^{\circ} 58^{\prime} 42^{\prime \prime} \mathrm{N}^{\circ} 53^{\prime} \\
22^{\prime \prime} \mathrm{W} 680\end{array}$ & SLK & Holocene & Total & Mariseal 1993 & MD-JC & 1994 \\
\hline 38 & Gallocanta (Teruel & $\begin{array}{l}40^{\circ} 59^{\prime} 36^{\prime \prime} \mathrm{N} 1^{\circ} \\
30^{\prime} 31^{\prime \prime} \mathrm{W} 995\end{array}$ & SLK & c. Last $12000 \mathrm{BP}$ & Partial & Burjachs et al. 1996 & $\mathrm{FB}$ & 1994 \\
\hline 39 & Juncabalejo (Huelva) & $\begin{array}{l}36^{\circ} 56^{\prime} 10^{\prime \prime} \mathrm{N} 6^{\circ} 22^{\prime} \\
56^{\prime \prime} \mathrm{W} 0\end{array}$ & SLK & Sub-recent & Partial & Yáñez $\underline{2005}$ & CY & $2001-4$ \\
\hline 40 & La Playa (Zaragoza) & $\begin{array}{l}41^{\circ} 25^{\prime} 00^{\prime \prime} \mathrm{N}^{\circ} 11^{\prime} \\
10^{\prime \prime} \mathrm{W} 340\end{array}$ & SLK & $<9900$ cal BP & Partial & $\begin{array}{l}\text { González-Sampériz et } \\
a l .2008\end{array}$ & PGS & $2003-4$ \\
\hline \multirow{2}{*}{41} & \multirow{2}{*}{ La Salineta (Zaragoza) } & \multirow{2}{*}{$\begin{array}{l}41^{\circ} 28^{\prime} 55^{\prime \prime} \mathrm{N}^{\circ} 9^{\prime} \\
30^{\prime \prime} \mathrm{W} 340\end{array}$} & \multirow{2}{*}{ SLK } & $<7740 \mathrm{BP}$ & \multirow{2}{*}{ Partial } & $\begin{array}{l}\text { Valero-Garcés et al. } \\
2004\end{array}$ & $\mathrm{BD}, \mathrm{ACS}$ & 1994 \\
\hline & & & & Last $2000 \mathrm{BP}$ & & $\begin{array}{l}\text { González-Sampériz et } \\
\text { al. } 2008\end{array}$ & PGS & $2003-4$ \\
\hline 42 & $\begin{array}{l}\text { Laguna Redonda } \\
\text { (Huelva) }\end{array}$ & $\begin{array}{l}37^{\circ} 12^{\prime} 13^{\prime \prime} \mathrm{N} 6^{\circ} 50^{\prime} \\
26^{\prime \prime} \mathrm{W} 36\end{array}$ & SLK & Holocene & Partial & $\begin{array}{l}\text { Stevenson } \\
\text { (unpublished) }\end{array}$ & ACS, BD & - \\
\hline 43 & Las Nuevas (Huelva) & $\begin{array}{l}36^{\circ} 50^{\prime} 30^{\prime \prime} \mathrm{N} 6^{\circ} 23^{\prime} \\
16^{\prime \prime} \mathrm{W} 1\end{array}$ & SLK & $\begin{array}{l}\text { Upper Pleistocene- } \\
\text { Holocene }\end{array}$ & Partial & - & IC & 2007 \\
\hline 44 & Mari López (Huelva) & $\begin{array}{l}37^{\circ} 1^{\prime} 4^{\prime \prime} \mathrm{N} 6^{\circ} 18^{\prime \prime} \\
33^{\prime \prime} \mathrm{W} 0\end{array}$ & SLK & Last $4000 \mathrm{BP}$ & Partial & YIl et al. 2004 & RY & 2003 \\
\hline 45 & $\begin{array}{l}\text { Mediana de Aragón } \\
\text { (Zaragoza) }\end{array}$ & $\begin{array}{l}41^{\circ} 30^{\prime} 10^{\prime \prime} \mathrm{N} 0^{\circ} 44^{\prime} \\
00^{\prime \prime} \text { W } 340\end{array}$ & SLK & $14000-10000 \mathrm{BP}$ & Partial & $\begin{array}{l}\text { Valero-Garcés et al. } \\
2000 \mathrm{a}, 2000 \mathrm{~b}\end{array}$ & PGS & $1996-8$ \\
\hline 46 & Medina (Huelva) & $\begin{array}{l}37^{\circ} 9^{\prime} 34^{\prime \prime} \mathrm{N} 6^{\circ} 50^{\prime} \\
28^{\prime \prime} \mathrm{W} 10\end{array}$ & SLK & Last $9000 \mathrm{BP}$ & Partial & Reed et al. 2001 & ACS & - \\
\hline 47 & Membrillo (Huelva) & $\begin{array}{l}36^{\circ} 53^{\prime} 10^{\prime \prime} \mathrm{N} 6^{\circ} 21^{\prime} \\
56^{\prime \prime} \mathrm{W} 4\end{array}$ & SLK & Sub-recent & Partial & Yáñez 2005 & CY & $2001-4$ \\
\hline 48 & Ontalafia (Albacete) & $\begin{array}{l}38^{\circ} 44^{\prime} 59^{\prime \prime} \mathrm{N} 1^{\circ} 46^{\prime} \\
49^{\prime \prime} \mathrm{W} 878\end{array}$ & SLK & Holocene & Total & Cirujano 1990 & MD-JC & 1994 \\
\hline
\end{tabular}


Table 4. Reported cases of palynological sterility in lacustrine sites. Continuation.

\begin{tabular}{|c|c|c|c|c|c|c|c|c|}
\hline $\begin{array}{c}\text { Site } \\
\text { Number }\end{array}$ & Site (province) & $\begin{array}{c}\text { Coordinates } \\
\text { Altitude } \mathrm{m} \text { asl }\end{array}$ & $\begin{array}{l}\text { Site } \\
\text { type }\end{array}$ & Age & Sterility & Reference & Analvst & $\begin{array}{l}\text { Lab } \\
\text { year }\end{array}$ \\
\hline 49 & Pétrola (Albacete) & $\begin{array}{l}38^{\circ} 50^{\prime} 28^{\prime \prime} \mathrm{N}^{\circ} 33^{\prime} \\
58^{\prime \prime} \mathrm{W} 854\end{array}$ & SLK & Holocene & Total & Cirujano 1990 & MD-JC & $1994-5$ \\
\hline 50 & Salines (Alacant) & $\begin{array}{l}38^{\circ} 30^{\prime} 02^{\prime \prime} \mathrm{N} 0^{\circ} 53^{\prime} \\
18^{\prime \prime} \mathrm{W} 471\end{array}$ & SLK & $\begin{array}{l}\text { Upper Pleistocene- } \\
\text { Holocene }\end{array}$ & Partial & $\begin{array}{l}\text { Julià et al. } 1994 \mathrm{~b}: \\
\text { Giralt et al. } 1999\end{array}$ & FB & 1992 \\
\hline 51 & Vetalengua (Huelva) & $\begin{array}{l}36^{\circ} 55^{\prime} 27^{\prime \prime} \mathrm{N} 6^{\circ} 22^{\prime} \\
29^{\prime \prime} \mathrm{W} 4\end{array}$ & SLK & $2300-<1700 \mathrm{BP}$ & Partial & Yáñez 2005 & CY & $2001-4$ \\
\hline 52 & Villardón (Zamora) & $\begin{array}{l}41^{\circ} 47^{\prime} 42^{\prime \prime} \text { N } 5^{\circ} 38^{\prime} \\
19^{\prime \prime} \text { W } 686\end{array}$ & SLK & $4150-3950 \mathrm{BP}$ & Partial & $\begin{array}{l}\text { Gómez-Ferreras et al. } \\
1996\end{array}$ & JLS & 1995 \\
\hline 53 & Xäbia (Alicante) & $\begin{array}{l}38^{\circ} 47^{\prime} 34^{\prime \prime} \mathrm{N} 0^{\circ} 10^{\prime} \\
02^{\prime \prime} \mathrm{W} 50\end{array}$ & SLK & Holocene & Partial & Viñals et al. 1993 & MD & 1993 \\
\hline
\end{tabular}

rreiras (Santos et al. 2000; Santos 2004), and two metres of organic clay from Laguna de Orcera were fully barren, with characteristics similar to Siles (Carrión 2002a), Cañada de la Cruz (Carrión et al. 2001c), and El Sabinar (Carrión et al. 2004a), which had provided high-quality pollen data to reconstruct the past vegetation changes in the Segura Mountains since the Last Glacial Maximum (Carrión 2002a). In Sierra de Gádor (Almería), a 150cm-depth core of red clay from Balsa del Sabinar was completely sterile, while a darker, more organic, lacustrine deposit formed under more permanent-water conditions was evenly polliniferous (Carrión et al. 2003a).

Palaeo-lakes deserve special attention because they are sometimes associated with palaeontological and palaeoanthropological excavations. Considerable effort, involving up to five trials, has been put into the famous enclaves of the GuadixBaza depression, without success (Gibert et al. 1988; Agustí and Julià 1990; Palmqvist et al. 2003) (Table 4). Repeated analyses probably resulted from the suggestion of an early Pleistocene (1.4-1.1 Ma) Eurasian colonisation by humans on the basis of local Oldowan and Acheulean lithics (Oms et al. 2000). Excavation sediments, although extremely rich in fauna, were also lacking pollen in the Plio-Pleistocene sites of Fonelas and Mencal, in the same basin. Sediments at these sites are very diverse including clays, silts and sands, occasionally exposing micrite limestone layers formed on the margins of palaeo-lacustrine depositional environments under low-energy water conditions (Arribas et al. 2004b). Other palaeolacustrine records, now in an exposed situation, have shown partially polliniferous results, such as Linás in Huesca, where Martí et al. (2002) and González-Sampériz et al. (2005) have identified evidence of selective pollen corrosion.

Saline lakes, widespread in endorheic depressions of inland Iberia (González-Beserán et al. 1991; Casado and Montes 1995) have also presented difficulties, especially when shortlived or seasonal (Table 4, Fig. 2). A notorious case study concerns the Pétrola, El Acequión and Ontalafia lakes in La Mancha Plain of south-central Spain. An international project (PB91-0897, MEC 1992-95) led by M. Dupré, University of Valencia (Table 2), had been specifically designed to link environmental and cultural changes during the Holocene, based on the pollen records expected from these lakes and archaeo- logical reports from adjacent settlement sites (Nájera and Molina 1977; Jordán 1992). Deep sediment cores of 2900, 1010 and $1350 \mathrm{~cm}$ were extracted from Pétrola, El Acequión, and Ontalafia, respectively. A total of 90 (Pétrola), 53 (Acequión), and 65 (Ontalafia) sediment samples were processed in the palynological laboratories of Valencia (MD) and Murcia (JC), but they all failed to show pollen.

Coastal salt marshes and lagoons, while equally problematic, represent a risk worth taking for pollen analysis (Table 1). A multi-core approach is appropriate here because pollen corrosion and sedimentary and palynological hiatuses may affect the deposit unevenly across the basin (Table 4). Successful cases come from the most arid parts of eastern Spain. Coastal salt marshes of San Rafael, Roquetas de Mar, and Antas (Almería) have yielded pollen records from pleniglacial to late Holocene times (Yll et al. 1994; Pantaleón-Cano et al. 2003). Northwards in Alicante, the Elx pollen sequence was also obtained from a lagoon (Burjachs et al. 2000). Pollen analyses of two coreholes in the Pego-Oliva littoral marsh were less rewarding. Pollen was scarce, poorly preserved, and episodically absent from quite an organic-rich, yet salty, shallow-sea sediment (Table 4).

Pollen analyses carried out in the Doñana marshlands (Abalario Estuaries Complex in the coastal arc of Huelva between the Guadalquivir and Tinto deltas) (Fig. 1) have produced contrasting results. In general, organic-rich layers are polliniferous (Table 4). Some marshy sediments have given pollen records, such as Las Madres (Stevenson 1985), El Acebrón (Stevenson and Moore 1988), Mari López (Yll et al. 2003), Laguna Redonda and Línea de la Mediana (Stevenson n.d.), and Las Nuevas. Other 'marisma' deposits have not been as rewarding, such as Carrizosa, Cherri, Juncabalejo, Membrillo, and Vetalengua (Yáñez 2005; Yáñez et al. 2006).

\subsection{Caves and rockshelters}

A number of archaeological caves with excavated profiles show pollen-sterile layers, or sometimes whole sterile sections, even after several trials (Table 5). Well-known sites of the Pleistocene include the Mousterian Cova Negra of Xativa (Fumanal 1986), Cova de les Cendres (Dupré 1988; Badal and Carrión 2001), Cova de El Salt (Fumanal 1986), Cueva de Nerja's 
Table 5. Cases of palynological sterility with caves $(C)$, and rockshelters (R).

\begin{tabular}{|c|c|c|c|c|c|c|c|c|}
\hline $\begin{array}{c}\text { Site } \\
\text { Number } \\
\end{array}$ & Site & $\begin{array}{l}\text { Coordinates } \\
\text { Altitude m asl }\end{array}$ & Site & Age/Industry & Sterility & Reference & Analust & $\begin{array}{l}\text { Lab } \\
\text { Year }\end{array}$ \\
\hline 54 & $\begin{array}{l}\text { A Valiña } \\
\text { (Orense) }\end{array}$ & $\begin{array}{l}43^{\circ} 2^{\prime} 27^{\prime \prime} \mathrm{N} 7^{\circ} 19^{\prime} \\
4^{\prime \prime} \text { W } 550\end{array}$ & c & Middle-Upper Palaeolithic & Partial & $\begin{array}{l}\text { Fernández-Rodriguez et } \\
a l .1995\end{array}$ & PRR & - \\
\hline 55 & $\begin{array}{l}\text { Altamira } \\
\text { (Cantabria) }\end{array}$ & $\begin{array}{l}43^{\circ} 22^{\prime} 57^{\prime \prime} \mathrm{N} 4^{\circ} \\
06^{\prime} 58^{\prime \prime} \text { W } 75\end{array}$ & c & Magdalenian & Partial & $\begin{array}{l}\text { Lasheras and de las } \\
\text { Heras } 1997\end{array}$ & MD JC & 1997 \\
\hline 56 & $\begin{array}{l}\text { Amalda } \\
\text { (Guipúzcoa) }\end{array}$ & $\begin{array}{l}43^{\circ} 14^{\prime} 6^{\prime \prime} \mathrm{N} 2^{\circ} 13^{\prime} \\
37^{\prime \prime} \mathrm{W} 220^{\prime}\end{array}$ & c & $\begin{array}{l}\text { Mousterian Solutrean } \\
\text { Chalcolithic }\end{array}$ & Partial & Altuna et al. 1990 & MD & 1984 \\
\hline 57 & $\begin{array}{l}\text { Atapuerea Sima } \\
\text { Huesos (Burgos) }\end{array}$ & $\begin{array}{l}42^{\circ} 21^{\prime} 6^{\prime \prime} \mathrm{N}^{\circ} 31^{\prime} \\
12^{\prime \prime} \mathrm{W} 994\end{array}$ & c & Middle Pleistocene & Partial & Garcia-Antón 1987 & MGA & $1984-7$ \\
\hline 58 & $\begin{array}{l}\text { Atapuerca Galeria } \\
\text { (Burgos) }\end{array}$ & $\begin{array}{l}42^{\circ} 21^{\prime} 5^{\prime \prime} \mathrm{N} 3^{\circ} 31^{\prime} \\
11^{\prime \prime} \mathrm{W} 999\end{array}$ & c & Middle Pleistocene & Partial & $\begin{array}{l}\text { García-Antón et al. } \\
\text { 1990; Garcia-Antón and } \\
\text { Sainz-Ollero } 1991\end{array}$ & MGA & $1984-7$ \\
\hline \multirow[t]{2}{*}{59} & \multirow{2}{*}{$\begin{array}{l}\text { Atapuerea Gran } \\
\text { Dolina (Burgos) }\end{array}$} & \multirow{2}{*}{$\begin{array}{l}42^{\circ} 21^{\prime} 6^{\prime \prime} \mathrm{N}^{\circ} 31^{\prime} \\
12^{\prime \prime} \text { W } 994\end{array}$} & \multirow[t]{2}{*}{ c } & \multirow[t]{2}{*}{ Lower-Middle Pleistocene } & \multirow[t]{2}{*}{ Partial } & $\begin{array}{l}\text { Garcia-Antón 1995; } \\
\text { Cattani et al. } 1994\end{array}$ & MGA & \multirow[t]{2}{*}{$1984-7$} \\
\hline & & & & & & Burjachs 2001 & FB & \\
\hline 60 & $\begin{array}{l}\text { Atapuerca Tres } \\
\text { Simas (Burgos) }\end{array}$ & $\begin{array}{l}42^{\circ} 2 \mathrm{I}^{\prime} 5^{\prime \prime} \mathrm{N}^{\circ} 31^{\prime} \\
1^{\prime \prime} \text { W } 999^{\circ}\end{array}$ & c & Middle Pleistocene & Total & Garcia-Antón 1989 & MGA & $1984-7$ \\
\hline 61 & $\begin{array}{l}\text { Berroberria } \\
\text { (Navarra) }\end{array}$ & $\begin{array}{l}43^{\circ} 16^{\circ} 43.05^{\prime \prime} \mathrm{N} \\
1^{\circ} 31^{\prime} 34.64^{\prime \prime} \mathrm{W} 160\end{array}$ & c & Magdalenian-Neolithic & Partial & Boyer-Klein 1988 & BK & - \\
\hline 62 & \begin{tabular}{|l} 
Bolomor \\
(Valencia)
\end{tabular} & $\begin{array}{l}39^{\circ} 4^{\prime} 48^{\prime \prime} \mathrm{N} 0^{\circ} 16^{\prime} \\
59^{\prime \prime} \text { W } 22\end{array}$ & $\begin{array}{l}\text { C (be. } \\
\text { bt) }\end{array}$ & Mousterian $525-121 \mathrm{ka}$ & Total & Fernández-Peris 2004 & MD & $1994-5$ \\
\hline 63 & $\begin{array}{l}\text { Bolumini } \\
\text { (Alicante) }\end{array}$ & $\begin{array}{l}38^{\circ} 50^{\prime \prime} 13^{\prime \prime} \mathrm{N} 0^{\circ} \\
00^{\circ} 50^{\prime \prime} \mathrm{W} 170\end{array}$ & C & Neolithic & Partial & Sanchis $\underline{1992}$ & AKS & 1990 \\
\hline 64 & $\begin{array}{l}\text { Calaveres } \\
\text { (Alicante) }\end{array}$ & $\begin{array}{l}38^{\circ} 47^{\prime} 37^{\prime \prime} \mathrm{N}^{\circ} 1^{\prime} \\
43.56^{\prime \prime} \mathrm{W} 180\end{array}$ & c (be) & Mousterian & Partial & Vives 1982 & MD & 1982 \\
\hline 65 & $\begin{array}{l}\text { Carihuela } \\
\text { (Granada) }\end{array}$ & $\begin{array}{l}37^{\circ} 26^{\prime} 22^{\prime \prime} \mathrm{N}^{\circ} \\
26^{\circ} 13^{\prime \prime} \mathrm{W} 1078\end{array}$ & c & Last $120 \mathrm{ka}$ & Partial & Carrión 1992b & JC & $\begin{array}{l}1988- \\
1999\end{array}$ \\
\hline 66 & $\begin{array}{l}\text { Cau del Duc } \\
\text { d'Ullà (Girona) }\end{array}$ & $\begin{array}{l}42^{\circ} 3^{\prime} 51^{\prime \prime} \mathrm{N} 3^{\circ} 7 \\
39^{\prime \prime} \mathrm{E} 135\end{array}$ & $C(b c)$ & Pleistocene & Total & Guilbaud et al. 1993 & FB & 1978 \\
\hline 67 & $\begin{array}{l}\text { Cendres } \\
\text { (Alicante) }\end{array}$ & $\begin{array}{l}38^{\circ} 41^{\prime} 10^{\prime \prime} \mathrm{N}^{\circ} 9^{\prime} \\
9^{\prime \prime} \mathrm{E} 35\end{array}$ & $\mathrm{C}$ (be) & $24-6 \mathrm{ka}$ & Partial & Villaverde $\underline{2001}$ & MD & 1992 \\
\hline 68 & $\begin{array}{l}\text { Cova } 120 \\
\text { (Girona) }\end{array}$ & $\begin{array}{l}42^{\circ} 16^{\prime} 30^{\prime \prime} \mathrm{N} 2^{\circ} \\
36^{\circ} 42^{\prime \prime} \mathrm{E} 460\end{array}$ & c & Middle Palaeolithic & Partial & Burjachs 1988a & FB & 1983 \\
\hline 69 & \begin{tabular}{|l} 
Cova Beneito \\
(Alicante)
\end{tabular} & $\begin{array}{l}38^{\circ} 48^{\prime} 7^{\prime \prime} \mathrm{N} 0^{\circ} 28^{\prime} \\
26^{\prime \prime} \mathrm{W} 650\end{array}$ & c & $\begin{array}{l}\text { Mousterian-Upper } \\
\text { Paleolithic }>38-16 \mathrm{ka}\end{array}$ & Partial & Carrión 1992a & JC & $\begin{array}{l}1989- \\
1996\end{array}$ \\
\hline 70 & $\begin{array}{l}\text { Cova dEn Pardo } \\
\text { (Alicante) }\end{array}$ & $\begin{array}{l}38^{\circ} 44^{\prime} 04^{\prime \prime} \mathrm{N}^{\circ} \\
26^{\prime} 10^{\prime \prime} \mathrm{W} 500\end{array}$ & c & Neolithie/Bronze & Partial & $\begin{array}{l}\text { Dupré êt al. } 1999: \\
\text { González-Sampériz } 1999\end{array}$ & $\begin{array}{l}\text { MD } \\
\text { PGS }\end{array}$ & 1997 \\
\hline \multirow[t]{2}{*}{71} & \multirow{2}{*}{$\begin{array}{l}\text { Cova Fosca } \\
\text { (Castellón) }\end{array}$} & \multirow{2}{*}{$\begin{array}{l}40^{\circ} 27^{\prime} 25^{\prime \prime} \mathrm{N} 0^{\circ} 8^{\prime} \\
0^{\prime \prime} \text { W } 1150\end{array}$} & \multirow{2}{*}{$\mathrm{C}(\mathrm{bt})$} & \multirow{2}{*}{ Meso-Neolithic } & \multirow{2}{*}{ Total } & Yll 1988 & IP & \multirow{2}{*}{1980} \\
\hline & & & & & & Cebrià et al. 1988 & RY & \\
\hline \multirow{2}{*}{72} & \multirow{2}{*}{$\begin{array}{l}\text { Cova Matutano } \\
\text { (Castellón) }\end{array}$} & \multirow{2}{*}{$\begin{array}{l}40^{\circ} 6^{\prime} 46^{\prime \prime} \mathrm{N}^{\circ} 3^{\prime} \\
11^{\prime \prime} \mathrm{W} 324\end{array}$} & \multirow{2}{*}{ C } & \multirow{2}{*}{$\begin{array}{l}\text { Magdalenian 13960- } \\
11410 \mathrm{BP}\end{array}$} & Total & YII $\underline{1983}$; Burjachs $\underline{1999}$ & FB & $\begin{array}{l}1980- \\
86\end{array}$ \\
\hline & & & & & Partial & Yll et al. 2008d & $\begin{array}{l}\text { RY-FB- } \\
\text { IE }\end{array}$ & 2007 \\
\hline 73 & $\frac{\text { Cova Negra }}{\text { (Alicante) }}$ & $\begin{array}{l}38^{\circ} 39^{\prime} 8^{\prime \prime} \mathrm{N}^{\circ} 44^{\prime} \\
21^{\prime \prime} \mathrm{W} 911\end{array}$ & $\begin{array}{l}\mathrm{C}(\mathrm{hrt}, \\
\mathrm{bc}, \mathrm{bt})\end{array}$ & Mousterian c. $117.50 \mathrm{ka}$ & Total & Fumanal $\underline{1986}$ & MD & 1985 \\
\hline 74 & $\begin{array}{l}\text { Cueva del Calor } \\
\text { (Murcia) }\end{array}$ & $\begin{array}{l}38^{\circ} 5^{\prime} 18^{\prime \prime} \mathrm{N} \mathrm{I}^{\circ} 48^{\prime} \\
27^{\prime \prime} \mathrm{W} 670\end{array}$ & $c(b t)$ & Meso-Neolithic & Partial & López-Garcia 1988 & PLG & 1986 \\
\hline 75 & $\begin{array}{l}\text { Cova de l'Or } \\
\text { (Alicante) }\end{array}$ & $\begin{array}{l}38^{\circ} 49^{\prime} 344^{\prime \prime} 0^{\circ} \\
23^{\circ} 04^{\prime \prime} \mathrm{W} 400\end{array}$ & C & Neolithic & Partial & Duprê et al. $\underline{1983}$ & MD & 1980 \\
\hline 76 & $\begin{array}{l}\text { Cueva Tomás } \\
\text { (Granada) }\end{array}$ & $\begin{array}{l}37^{\circ} 44^{\prime} 19^{\prime \prime} \mathrm{N} 2^{\circ} \\
26^{\prime} 5^{\prime \prime} \mathrm{W} 970\end{array}$ & c & Lower Pleistocene & Total & Agusti \& Julià 1990 & SL & $\begin{array}{l}\text { Late } \\
1980 \text { s }\end{array}$ \\
\hline 77 & Chaves (Huesca) & $\begin{array}{l}42^{\circ} 12^{\prime} 48^{\prime \prime} \mathrm{N}^{\circ} 0^{\circ} 8^{\prime} \\
30^{\prime \prime} \mathrm{W} 663\end{array}$ & c & $\begin{array}{l}\text { Upper Palaeolithic- } \\
\text { Bronze Age }\end{array}$ & Partial & Castản \& Baldellou 1985 & PGS & 1998 \\
\hline 78 & $\begin{array}{l}\text { Ekain } \\
\text { (Guipúzeoa) }\end{array}$ & $\begin{array}{l}43^{\circ} 14^{\prime} 18^{\prime \prime} \mathrm{N} 2^{\circ} \\
16^{\circ} 09^{\prime \prime} \mathrm{W} 90\end{array}$ & c & Magdalenian/Azilian & Partial & Altuma \& Merino 1984 & MD & 1988 \\
\hline 79 & $\begin{array}{l}\text { El Ángel } \\
\text { (Córdoba) }\end{array}$ & $\begin{array}{l}37^{\circ} 24^{\prime} 31^{\prime \prime} \mathrm{N} 4^{\circ} \\
29^{\prime} 8^{\prime \prime} \mathrm{W} 500\end{array}$ & c & Mid-Upper Pleistocene & Total & Botella et al. 2006 & JC & 2004 \\
\hline 80 & El Arca (Cádiz) & $\begin{array}{l}36^{\circ} 45^{\prime} 30^{\prime \prime} \mathrm{N} 5^{\circ} \\
21^{\prime} 57^{\prime \prime} \text { W } 850\end{array}$ & c & - & Total & Acosta 1968 & JLS & 1998 \\
\hline 81 & $\begin{array}{l}\text { El Castillo } \\
\text { (Cantabria) }\end{array}$ & $\begin{array}{l}43^{\circ} 17^{\prime} 55^{\prime \prime} \mathrm{N} 3^{\circ} \\
57^{\circ} 43^{\prime \prime} \text { W } 75\end{array}$ & c & Last $120 \mathrm{ka}$ & Total & $\begin{array}{l}\text { Bemaldo de Quirós \& } \\
\text { Cabrera } 2000\end{array}$ & JLS & 1983 \\
\hline 82 & $\begin{array}{l}\text { El Moro de } \\
\text { Olvena (Huesea) }\end{array}$ & $\begin{array}{l}42^{\circ} 6^{\circ} 48^{\prime \prime} \mathrm{N} 0^{\circ} 17^{\prime} \\
25^{\prime \prime} \mathrm{E} 380\end{array}$ & c & Holocene & Total & $\begin{array}{l}\text { Alday 1995; Cuchi \& } \\
\text { Sancho 1995 }\end{array}$ & PGS & 1988 \\
\hline 83 & $\begin{array}{l}\text { El Pendo } \\
\text { (Cantabria) }\end{array}$ & $\begin{array}{l}43^{\circ} 24^{\prime} 17^{\prime \prime} \mathrm{N} 3^{\circ} \\
54^{\prime} 2^{\prime \prime} \text { W } 51\end{array}$ & c & Lateglacial-Boreal & Partial & Leroi-Gourhan $\underline{1980}$ & ALG & - \\
\hline 84 & $\begin{array}{l}\text { Erralla } \\
\text { (Guipúzcoa) }\end{array}$ & $\begin{array}{l}43^{\circ} 11^{\prime} 51^{\prime \prime} 2^{\circ} \\
13^{\prime} 36 \mathrm{~W} 500\end{array}$ & c & Paleolithic & Partial & Altuna et al. 1985 & BK & $1980 \mathrm{~s}$ \\
\hline 85 & $\frac{\text { La Blanca }}{\text { (Murcia) }}$ & $\begin{array}{l}38^{\circ} 9^{\prime} 27^{\prime \prime} \mathrm{N}^{\circ} 2 \mathrm{I}^{\prime} \\
36^{\prime \prime} \mathrm{W} 300\end{array}$ & C (sp) & Pleistocene & Total & - & IC & 1999 \\
\hline 86 & $\begin{array}{l}\text { Labeko } \\
\text { (Guipuzcoa) }\end{array}$ & $\begin{array}{l}43^{\circ} 3^{\prime} 36^{\prime \prime} \mathrm{N} 2^{\circ} 29^{\prime} \\
25^{\prime \prime} \mathrm{W} 260\end{array}$ & c & $\begin{array}{l}\text { Aurignacian- } \\
\text { Chatelperronian }\end{array}$ & Partial & Sảnchez-Goùi 1991 & MFSG & - \\
\hline 87 & $\begin{array}{l}\text { Les Malladetes } \\
\text { (Valencia) }\end{array}$ & $\begin{array}{l}39^{\circ} 00^{\prime} 51^{\prime \prime} \mathrm{N} 0^{\circ} \\
17^{\prime} 41^{\prime \prime} \mathrm{W} 5000\end{array}$ & c & Upper Palaeolithic & Partial & Fortea \& Jordá 1976 & MD & 1988 \\
\hline 88 & $\begin{array}{l}\text { Lezetxiki } \\
\text { (Guipuzcoa) }\end{array}$ & $\begin{array}{l}43^{\circ} 4^{\prime} 51^{\prime \prime} \mathrm{N} 2^{\circ} 31^{\prime} \\
49^{\prime \prime} \mathrm{W} 350^{\prime}\end{array}$ & c & Mousterian-Bronze & Partial & Sánchez-Goùi 1991 & MFSG & - \\
\hline 89 & $\begin{array}{l}\text { Los Azules } \\
\text { (Asturias) }\end{array}$ & $\begin{array}{l}43^{\circ} 20^{\prime} 55^{\prime \prime} \mathrm{N} 5^{\circ} 7^{\prime} \\
37^{\prime \prime} \mathrm{W} 200\end{array}$ & c & Azilian $11000-9430 \mathrm{BP}$ & Partial & López-Garcia 1981 & PLG & 1980 \\
\hline
\end{tabular}


Table 5. Cases of palynological sterility with caves (C), and rockshelters (R). Continuation.

\begin{tabular}{|c|c|c|c|c|c|c|c|c|}
\hline Site & Site & $\begin{array}{l}\text { Coordinates } \\
\text { Altitude } \mathrm{m} \text { asl }\end{array}$ & Site & Age/Industry & Sterility & Reference & Analvst & $\begin{array}{l}\text { Lab } \\
\text { Year }\end{array}$ \\
\hline \multirow{3}{*}{90} & \multirow{3}{*}{$\begin{array}{l}\text { Mazaculos } \\
\text { (Asturias) }\end{array}$} & \multirow{3}{*}{$\begin{array}{l}43^{\circ} 23^{\prime} 22^{\prime \prime} \mathrm{N} 4^{\circ} \\
34^{\prime} 55^{\prime \prime} \text { W } 70\end{array}$} & \multirow{3}{*}{ c } & $9290 \mathrm{BP}$ & Partial & López-Garcia 1986 & PLG & 1983 \\
\hline & & & & Preboreal-Boreal & Partial & López-Garcia 1986 & JS & 1983 \\
\hline & & & & Preboreal-Boreal & Total & $\begin{array}{l}\text { González-Morales et al. } \\
1980\end{array}$ & KV & - \\
\hline 91 & $\begin{array}{l}\text { Nacimiento } \\
\text { (Jaén) }\end{array}$ & $\begin{array}{l}38^{\circ} 6^{\prime} 18^{\prime \prime} \mathrm{N} 2^{\circ} 41^{\prime} \\
36^{\prime \prime} \text { W } 1600\end{array}$ & $C(b t)$ & Neolithic & Partial & $\begin{array}{l}\text { Asquerino \& López- } \\
\text { Gareia } 1981\end{array}$ & PLG & 1980 \\
\hline 92 & Nerja (Málaga) & $\begin{array}{l}36^{\circ} 45^{\prime} 42^{\prime \prime} \mathrm{N}^{\circ} \\
52^{\prime} 6^{\prime \prime} \mathrm{W} 110\end{array}$ & $\begin{array}{l}\text { C (be, } \\
\text { hrt) }\end{array}$ & $\begin{array}{l}\text { Upper Pleistocene- } \\
\text { Holocene }\end{array}$ & Total & Arribas et al. 2004a & $\mathrm{MD}$ & 1986 \\
\hline 93 & $\begin{array}{l}\text { Palomas chasm } \\
\text { (Murcia) }\end{array}$ & $\begin{array}{l}37^{\circ} 47^{\prime} 54^{\prime \prime} \mathrm{N} 0^{\circ} \\
53^{\prime} 53^{\prime \prime} \mathrm{E} 60\end{array}$ & $\mathrm{C}(\mathrm{be})$ & $130-120 \mathrm{ka}$ & Total & Carrión et al. $2003 \mathrm{~b}$ & MD-JC & 2001 \\
\hline 94 & Pouảs (Ibiza) & 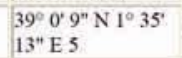 & c & c. $100 \mathrm{ka}$ & Partial & Guerrero \& Gomés 2000 & MGA & 1992 \\
\hline 95 & $\begin{array}{l}\text { Rascaño } \\
\text { (Cantabria }\end{array}$ & $\begin{array}{l}43^{\circ} 17^{\prime} 42^{\prime \prime} \mathrm{N} 3^{\circ} \\
42^{\prime} 10^{\prime \prime} \mathrm{W} 200\end{array}$ & C & Azilian 10558-10486 BP & Partial & Boyer-Klein 1981 & BK & - \\
\hline 96 & Sorbas (Almeria) & $\begin{array}{l}37^{\circ} 6^{\prime} 18^{\prime \prime} \mathrm{N} 2^{\circ} 4^{\prime} \\
38^{\prime \prime} \mathrm{W} 330\end{array}$ & C (gsp) & Pleistocene & Total & $\begin{array}{l}\text { Calaforra \& Pulido- } \\
\text { Boseh } 2003\end{array}$ & JC & 1999 \\
\hline 97 & $\begin{array}{l}\text { Tossal de la Font } \\
\text { (Castelló) }\end{array}$ & $\begin{array}{l}40^{\circ} 06^{\prime} 25.14^{\prime \prime} \mathrm{N} \\
0^{\circ} 03^{\prime} 17^{\prime \prime} \mathrm{W} 361\end{array}$ & c & Pleistocene & Total & Oläria et al. $\underline{2007}$ & FB-IE & 2003 \\
\hline 98 & $\begin{array}{l}\text { Valdegoba } \\
\text { (Burgos) }\end{array}$ & $\begin{array}{l}42^{\circ} 31^{\prime} 18^{\prime \prime} \mathrm{N} 3^{\circ} \\
46^{\prime} 16^{\prime \prime} \mathrm{W} 890\end{array}$ & C & Mousterian & Total & Diez et al. 1989 & MGA & 1989 \\
\hline 99 & Zatoya (Navarra) & $\begin{array}{l}42^{\circ} 54^{\prime} 2^{\prime \prime} \mathrm{N}^{\circ} 12^{\prime} \\
16^{\prime \prime} \mathrm{W} 1000\end{array}$ & C & Epipalaeolithic $8260 \mathrm{BP}$ & Partial & Boyer-Klein 1989 & BK & - \\
\hline 100 & $\begin{array}{l}\text { Abric Agut } \\
\text { (Barcelona) }\end{array}$ & $\begin{array}{l}41^{\circ} 31^{\prime} 50^{\prime \prime} \mathrm{N} 1^{\circ} \\
41^{\prime} 24^{\prime \prime} \text { E } 305\end{array}$ & $R$ (tr) & $\begin{array}{l}\text { Upper Pleistocene- } \\
\text { Holocene }\end{array}$ & Partial & Vaquero 2001 & FB & $1998-9$ \\
\hline 101 & $\begin{array}{l}\text { Abric del Filador } \\
\text { (Tarragona) }\end{array}$ & $\begin{array}{l}41^{\circ} 17^{\prime} 24^{\prime \prime} \mathrm{N} 0^{\circ} \\
45^{\circ} 17^{\prime \prime} \mathrm{E} 437\end{array}$ & $\mathrm{R}$ & $\begin{array}{l}\text { Upper Pleistocene- } \\
\text { Holocene }\end{array}$ & Total & $\begin{array}{l}\text { Garcia-Argüelles et al. } \\
2005\end{array}$ & AE-IP & 1983 \\
\hline 102 & $\begin{array}{l}\text { Abric Romani } \\
\text { (Barcelona) }\end{array}$ & $\begin{array}{l}41^{\circ} 31^{\prime} 57^{\prime \prime} \mathrm{N} 1^{\circ} \\
41^{\prime} 18^{\prime \prime} \text { E } 314\end{array}$ & $\mathrm{R}(\mathrm{tr})$ & Upper Pleistocene & Partial & Burjachs \& Julià 1994 & FB & $\begin{array}{l}1998- \\
2004\end{array}$ \\
\hline 103 & $\begin{array}{l}\text { Abrigo de Ángel } \\
\text { (Teruel) }\end{array}$ & $\begin{array}{l}40^{\circ} 44^{\prime} 31^{\prime \prime} \mathrm{N}^{\circ} \\
23^{\prime} 59^{\prime \prime} \mathrm{W} 700 \mathrm{~m}\end{array}$ & R & Holocene & Total & $\begin{array}{l}\text { Sebastián \& Zozaya } \\
1991\end{array}$ & PGS & 1997 \\
\hline 104 & $\begin{array}{l}\text { Abrigo Alejandre } \\
\text { (Soria) }\end{array}$ & $\begin{array}{l}41^{\circ} 20^{\prime} 30^{\prime \prime} \mathrm{N}^{\circ} \\
58^{\circ} 18^{\prime \prime} \mathrm{W} 960\end{array}$ & $\begin{array}{l}\mathrm{R}(\mathrm{bc}, \\
\mathrm{bt})\end{array}$ & Pleistocene & Total & Utrilla et al. 2000 & PGS & 2000 \\
\hline 105 & $\begin{array}{l}\text { Bauma dels } \\
\text { Pinyons } \\
\text { (Barcelona) }\end{array}$ & $\begin{array}{l}41^{\circ} 31^{\prime} 56^{\prime \prime} \mathrm{N} 1^{\circ} \\
41^{\prime} 20^{\prime \prime} \text { E } 310\end{array}$ & $\mathrm{R}(\mathrm{tr})$ & $\begin{array}{l}\text { Upper Pleistocene- } \\
\text { Holocene }\end{array}$ & Partial & Vaquero $\underline{2006}$ & FB & $1998-9$ \\
\hline 106 & $\begin{array}{l}\text { Costa d'En Manel } \\
\text { (Barcelona) }\end{array}$ & $\begin{array}{l}141^{\circ} 32^{\prime} 2^{\prime \prime} \mathrm{N}^{\circ} 41^{\prime} \\
25^{\prime \prime} \mathrm{E} 330\end{array}$ & $R$ (tr) & $\begin{array}{l}\text { Upper Pleistocene- } \\
\text { Holocene }\end{array}$ & Partial & - & FB & 2002 \\
\hline 107 & $\begin{array}{l}\text { Cueva Antón } \\
\text { (Murcia) }\end{array}$ & $\begin{array}{l}38^{\circ} 4^{\prime} 0^{\prime \prime} \mathrm{N} 1^{\circ} 29^{\prime \prime} \\
33^{\prime \prime} \mathrm{W} 370\end{array}$ & $\mathrm{R}$ & Mid-Upper Palacolithic & Total & Martinez-Sánchez 1992 & JC & 1995 \\
\hline 108 & El Salt (Alicante) & $\begin{array}{l}38^{\circ} 40^{\prime} 13^{\prime \prime} \mathrm{N}^{\circ} \\
21^{\prime} 52^{\prime \prime} \mathrm{W} 891\end{array}$ & $R$ (Ir) & Mousterian $60-40 \mathrm{ka}$ & Total & Fumanal 1986 & MD & 1988 \\
\hline 109 & $\begin{array}{l}\text { Entrefoces } \\
\text { (Oviedo) }\end{array}$ & $\begin{array}{l}43^{\circ} 3^{\prime} 36^{\prime \prime} \times 2^{\circ} 29^{\prime} \\
25^{\prime \prime} \text { W } 260\end{array}$ & R & Paleolithic & Total & Gonzảlez Morales $\underline{1990}$ & $\mathrm{MD}$ & $1980 \mathrm{~s}$ \\
\hline \multirow{2}{*}{110} & \multirow{2}{*}{\begin{tabular}{|l|} 
Forcas I \\
Forcas II \\
(Huesca)
\end{tabular}} & \multirow{2}{*}{$\begin{array}{l}42^{\circ} 11^{\prime} 33^{\prime \prime} \mathrm{N} 0^{\circ} \\
20^{\circ} 20^{\prime \prime} \text { E } 550\end{array}$} & $\mathrm{R}$ & $11300-9500 \mathrm{BP}$ & \multirow{2}{*}{ Total } & \multirow{2}{*}{ Utrilla \& Mazo 2008} & \multirow{2}{*}{ PGS } & \multirow{2}{*}{1998} \\
\hline & & & $R(b t)$ & $>8600 \mathrm{BP}$ & & & & \\
\hline 111 & $\begin{array}{l}\text { La Cativera } \\
\text { (Tarragona) }\end{array}$ & $\begin{array}{l}41^{\circ} 11^{\prime} 14^{\prime \prime} \mathrm{N} 0^{\circ} \\
20^{\circ} 6^{\prime \prime} \mathrm{E} 82\end{array}$ & $\mathrm{R}$ & Meso-Neolithic & Partial & $\begin{array}{l}\text { Allué \& Renault- } \\
\text { Miskovsky } 1999\end{array}$ & EA & 1988 \\
\hline 112 & $\begin{array}{l}\text { La Font Voltada } \\
\text { (Tarragona) }\end{array}$ & $\begin{array}{l}41^{\circ} 27^{\prime} 41^{\prime \prime} \mathrm{N} 1^{\circ} \\
1^{\prime} 58^{\prime \prime} \mathrm{E} 603\end{array}$ & $\mathrm{R}$ & Upper Pleistocene & Partial & $\begin{array}{l}\text { Burjachs \& Pérez-Obiol } \\
1989\end{array}$ & FB-RPO & 1988 \\
\hline 113 & $\begin{array}{l}\text { La Higuera } \\
\text { (Murcia) }\end{array}$ & $\begin{array}{l}37^{\circ} 34^{\prime} 59^{\prime \prime} \mathrm{N} 1^{\circ} \\
12^{\prime} 56^{\prime \prime} \text { W } 50\end{array}$ & $\mathrm{R}(\mathrm{bt})$ & Mesolithic & Total & $\begin{array}{l}\text { Martinez-Andreu \& } \\
\text { Sánchez } 2006\end{array}$ & JC & 1989 \\
\hline 114 & La Viña (Oviedo) & $\begin{array}{l}43^{\circ} 18^{\prime} 45^{\prime \prime} \mathrm{N} 5^{\circ} \\
49^{\prime} 41^{\prime \prime} \text { W } 220\end{array}$ & $R$ & Paleolithic & Total & Fortea 1990 & MD & $1980 \mathrm{~s}$ \\
\hline 115 & $\frac{\text { Legunova }}{\text { (Zaragoza) }}$ & $\begin{array}{l}42^{\circ} 21^{\prime} 21^{\prime \prime} \mathrm{N}^{\circ} \\
56^{\circ} 45^{\prime \prime} \mathrm{W} 700\end{array}$ & R & Azilian Meso-Neolithic & Partial & Montes $\underline{\underline{2005}}$ & PGS & 2004 \\
\hline 116 & Levante (Cádiz) & $\begin{array}{l}36^{\circ} 27^{\prime} 42^{\prime \prime} \mathrm{N} 5^{\circ} \\
55^{\circ} 42^{\prime \prime} \mathrm{W} 260\end{array}$ & $R$ & Upper Pleistocene & Total & Mas et al. 1998 & JLS & - \\
\hline 117 & $\begin{array}{l}\text { Los Aviones } \\
\text { (Murcia) }\end{array}$ & $\begin{array}{l}37^{\circ} 35^{\prime} 12^{\prime \prime} \mathrm{N}^{\circ} \\
59^{\prime} 13^{\prime \prime} \mathrm{W} 20\end{array}$ & $\begin{array}{l}\mathrm{R}(\mathrm{bc}, \\
\mathrm{bt})\end{array}$ & Middle Palaeolithic & Total & Montes $\underline{1987}$ & JC & 1987 \\
\hline 118 & $\begin{array}{l}\text { Los Baños } \\
\text { (Teruel) }\end{array}$ & $\begin{array}{l}41^{\circ} 2^{\prime} 5^{\prime \prime} \mathrm{N} 0^{\circ} 35^{\prime} \\
32^{\prime \prime} \text { W } 515\end{array}$ & $\mathrm{R}$ & $<7840 \mathrm{BP}$ & Partial & $\begin{array}{l}\text { González-Sampériz } \\
2004 \mathrm{~b}\end{array}$ & PGS & 2002 \\
\hline 119 & $\begin{array}{l}\text { Molino del } \\
\text { Vadico } \\
\text { (Albacete) }\end{array}$ & $\begin{array}{l}38^{\circ} 10^{\prime} 51^{\prime \prime} \mathrm{N} 2^{\circ} \\
26^{\circ} 58^{\prime \prime} \mathrm{W} 980\end{array}$ & $\begin{array}{l}\mathrm{R} \text { (bt, } \\
\mathrm{hrt} \text { ) }\end{array}$ & Last $12 \mathrm{ka}$ & Total & Vega-Toscano 1993 & IC & 1990 \\
\hline 120 & $\begin{array}{l}\text { Paco Pons } \\
\text { (Zaragoza) }\end{array}$ & $\begin{array}{l}42^{\circ} 21^{\prime} 21^{\prime \prime} \mathrm{N}^{\circ} \\
56^{\circ} 45^{\prime \prime} \mathrm{W} 1040\end{array}$ & R & Meso-Neolithic & Total & $\begin{array}{l}\text { Montes \& Domingo } \\
2001\end{array}$ & PGS & 2000 \\
\hline 121 & $\begin{array}{l}\text { Peña } 14 \\
\text { (Zaragoza) }\end{array}$ & $\begin{array}{l}42^{\circ} 21^{\prime} 21^{\prime \prime} \mathrm{N} 0^{\circ} \\
56^{\circ} 45^{\prime \prime} \text { W } 760\end{array}$ & R & Neolithic & Partial & $\begin{array}{l}\text { González-Sampériz et al. } \\
2005\end{array}$ & PGS & $2000-1$ \\
\hline 122 & $\begin{array}{l}\text { Peña del Diablo } \\
\text { (Zaragoza) }\end{array}$ & $\begin{array}{l}41^{\circ} 20^{\prime} 28^{\prime \prime} \mathrm{N} 1^{\circ} \\
58^{\prime} 14^{\prime \prime} \mathrm{W} 700\end{array}$ & R & $>11080 \mathrm{BP}$ & Partial & Utrilla ef al, 2000 & PGS & 1998 \\
\hline 123 & $\frac{\text { Ratlla del Bubo }}{\text { (Alicante) }}$ & $\begin{array}{l}38^{\circ} 16^{\prime} 48^{\prime \prime} \mathrm{N}^{\circ} \\
48^{\prime} 17^{\prime \prime} \mathrm{W} 200\end{array}$ & $\mathrm{R}$ (hrt) & Upper Palaeolithic & Total & Badal et al. 1990 & MD & 1991 \\
\hline 124 & $\begin{array}{l}\text { Tossal de la Roca } \\
\text { (Alicante) }\end{array}$ & $\begin{array}{l}38^{\circ} 47^{\prime} 18^{\prime \prime} \mathrm{N} 0^{\circ} \\
15^{\prime} 11^{\prime \prime} \mathrm{W} 650\end{array}$ & R & Lateglacial-Boreal & Partial & Cacho et al. 1995 & PLG & 1995 \\
\hline 125 & Vergara (Soria) & $\begin{array}{l}41^{\circ} 20^{\prime} 28^{\prime \prime} \mathrm{N} 1^{\circ} \\
58^{\prime} 14^{\prime \prime} \mathrm{W} 860\end{array}$ & $\mathrm{R}$ & Pleistocene-Holocene & Partial & Utrilla et al. 2000 & PGS & 1998 \\
\hline
\end{tabular}


Vestíbulo Chamber (Arribas et al. 2004a), Cueva de Altamira (Lasheras and De las Heras 1997), Cueva 120 (Agustí et al. 1987; Burjachs 1991a), Cueva del Castillo (Bernaldo de Quirós and Cabrera 2000), Cueva del Ángel, Pouás (Guerrero and Gornés 2000), Cueva de Amalda (Dupré 1988; Altuna et al. 1990), and noticeably, the mid-Pleistocene (350-120 ka) Cova de Bolomor, where 74 pollen samples from a $7 \mathrm{~m}$-deep stratigraphy were sterile (Fernández-Peris 2004).

Many of these cave stratigraphies include hearths, breccias, stalagmitic crusts, calcium-carbonate micelia, and more or less indurated strata, blocks, coarse-grain levels, lithics and other archaeological remains, bone remains, and shells. Hearths may or may not contain pollen. They did not in Civiacas (GonzálezSampériz 2001), Matutano (Burjachs 1999) and Filador (Burjachs 1999) and the Asturian cave of Los Azules (López-García 1981) (Table 5), but did in other Palaeolithic and Neolithic cave records (Dupré and Renault-Miskovsky 1990; Carrión and Dupré 2002; Carrión et al. 1999a; 2004b; 2008; López-Sáez et al. 2003; González-Sampériz 1998). The reason for this diversity of results is so far unknown. Cemented sediments coincide with sterility in Cau del Duc d'Ulla, Cueva de Valdegoba, Cova Fosca, Tossal de la Font, Cova Matutano, Bolomor, Cova Negra (Dupré 1988), Sima de las Palomas (Carrión et al. 2003b) and Cueva del Ángel (Table 5). In contrast, calcium carbonate deposits of Abric Romaní, Bauma dels Pinyons, Abric Agut and Costa d'En Manel rockshelters have provided pollen records for a major part of the Upper Pleistocene of north-eastern Iberia (Burjachs and Julià 1994; Allué et al. 1998; Burjachs 2000b). More general is the expected absence of pollen in sandy layers of cave stratigraphies, as seen in Cuevas de Levante (Cádiz), and Cueva de Chaves (Huesca) (Table 5). Several pollen sequences of the Cantabrian region are interrupted when reaching coarser-grain sediments: notably the caves of Lezetxiki and Labeko (Sánchez-Goñi 1991), Zatoya (Boyer-Klein 1989), and Berroberria (Boyer-Klein 1988).

Pollen analyses in the hominin-bearing Atapuerca (Burgos) have been rather unrewarding (García-Antón 1987; 1995; García-Antón et al. 1990; García-Antón and Sainz-Ollero 1991; Cattani et al. 1994; García-Antón and Casado 1994). M. García-Antón processed 84 samples from Galería levels TG-12 and TG-3, 87 samples from Gran Dolina levels TD-1 to TD-11, and 12 samples from Boca Norte chasm TN. All of them were palynologically sterile (Table 5). Other analysts, like F. Burjachs, who repeated analyses, have complained about the palynological poverty of Atapuerca.

Carihuela Cave (SE Spain) has proved useful for palaeoecological purposes: there are substantial pollen concentrations and a number of taxa, parallels between the curves of percentages and concentrations, ecological plausibility of the pollen spectra, and possibilities of correlation of pollen spectra from different sections of the same lithological units. Most profiles are, in fact, relatively rich in pollen, including Mousterian (Carrión 1992a), Upper Palaeolithic (Carrión et al. 1998) and Neolithic and Bronze Age levels (Fernández et al. 2007).
However, from the 12 lithostratigraphical units described by Vega-Toscano et al. (1988) for chambers CIII and CIV, both the unit XII and the lowermost levels of XI contained no pollen. There was a similar absence in unit VI in chamber CIII and CIV (Carrión et al. 1998). Although these deposits are the richest in organic content in the cave, it was clear that they had occasionally experienced repeated fluctuations of water levels (Vega-Toscano et al. 1988).

Pollen analyses in Cova Beneito present another interesting case study. This cave contains a continuous record of Middle Palaeolithic and Upper Palaeolithic industries, the latter extending from the Aurignacian to the Solutrean. In the course of excavations during 1990-91, Mousterian strata were polliniferous, but Upper Palaeolithic levels did not provide pollen from the available sections (Carrión 1992b). Later excavations exposed new profiles recording the whole sequence of Upper Palaeolithic industries. Surprisingly, these sediments proved to contain enough palynomorphs to undertake reliable pollen analysis (Carrión and Munuera 1997). Both profiles provided a stratigraphically coherent sequence involving the Middle and Upper Palaeolithic.

Sediments accumulated within rockshelters are prone to palynological sterility, often throughout the whole deposit (Table $\underline{1}$, Fig. 2). A considerable number of the failed records are rockshelters that show signs of burrowing activity by insects, earthworms, rootlets (Cueva de los Aviones, Cueva de la Higuera, Abrigo Alejandre, Abrigo del Molino del Vadico, Forcas II), and/or fluvial transport, flowing water or seepage (Abrigo de Angel, Forcas I, II, Legunova, Abrigo de los Baños de Ariño, Cueva de Antón) (Table 5). Again, sandy sediment is associated with sterility, as in Peña del Diablo, Legunova, Peña 14, and Abrigo del Filador (González-Sampériz 2004a; Utrilla et al. 2000; González-Sampériz et al. 2003a; 2005; García-Argüelles et al. 2005).

\subsection{Open-air archaeological and palaeontological sites, slope deposits, terraces, palaeosols and travertines}

Given their extraordinary abundance in the Iberian Peninsula since the Plio-Pleistocene, but especially after the Neolithic (Allué and Renault-Miskovsky 1999), the sediments associated with palaeontological, and archaeological open-air sites have traditionally been tested for palynology ( $\underline{\text { Table } 6}$ ). Reports of pollen occurrence in these sites, predominantly with coarse clasts and high lime concentration, are numerous in Spain (López-García 1991; Mariscal 1991a-c; 1992; Davis and Mariscal 1994; Castro et al. 1999; Fuentes et al. 2005; 2007; Postigo et al. 2007), but because of the high profile of some archaeological excavations, failures are highlighted.

Purely sandy and gravel-based layers are expected to be usually sterile or contain poor, contaminated, or non-significant pollen spectra. This is the case for the Chalcolithic sites of Los Molares (megalithic necropolis), Los Millares (Burjachs 
Table 6. Cases of palynological sterility with open-air archaeological sites. (OSA, mainly settlement sites) and palaeontological sites (OSP), exposed travertines (TR), palaeosoils (PL), fluvial terraces (T), slope deposits (SD), and moraine deposits (MD)

\begin{tabular}{|c|c|c|c|c|c|c|c|c|}
\hline$\frac{\text { Site }}{\text { Number }}$ & Site & $\begin{array}{c}\text { Coordinates Altitude } \\
\text { m asl }\end{array}$ & Type & Age/Industry & Sterility & Reference & Analvst & $\begin{array}{l}\text { Lab } \\
\text { Year }\end{array}$ \\
\hline 126 & Adra (Almeria) & $\begin{array}{l}36^{\circ} 44^{\prime} 53^{\prime \prime} \mathrm{N} 3^{\circ} 1^{\prime} \\
21^{\prime \prime} \mathrm{W} 3\end{array}$ & OSA & $2700-1800 \mathrm{bp}$ & Total & Suárez 1989 & JC & 1995 \\
\hline 127 & $\begin{array}{l}\text { Alcudia de Veo } \\
\text { (Castellón) }\end{array}$ & $\begin{array}{l}39^{\circ} 55^{\prime} 01^{\prime \prime} \mathrm{N}^{\circ} 21^{\prime} \\
21^{\prime \prime} \mathrm{W} 480\end{array}$ & OSA & Holocene & Partial & Butzer et al. $\underline{1986}$ & MD & 1988 \\
\hline 128 & $\begin{array}{l}\text { Bóbila Madurell } \\
\text { (Barcelona) }\end{array}$ & $\begin{array}{l}41^{\circ} 30^{\prime} 49^{\prime \prime} \mathrm{N} 2^{\circ} 06^{\prime} \\
03^{\prime \prime} \mathrm{E} 157\end{array}$ & OSA & Neolithic-Medieval & Partial & $\begin{array}{l}\text { Burjachs \& Pérez- } \\
\text { Obiol 1988 }\end{array}$ & FB-RPO & 1987 \\
\hline 129 & Buzanca I (Madrid) & $\begin{array}{l}40^{\circ} 10^{\prime} 18^{\prime \prime} \mathrm{N} 3^{\circ} 39^{\prime} \\
17^{\prime \prime} \mathrm{W} 600\end{array}$ & OSA & Chalcolithic & Total & López-Garcia 1997 & PLG & 1994 \\
\hline 130 & $\begin{array}{l}\text { Cabezo Redondo } \\
\text { (Alicante) }\end{array}$ & $\begin{array}{l}38^{\circ} 38^{\prime} 43^{\prime \prime} \mathrm{N} 0^{\circ} 53^{\prime} \\
34^{\prime \prime} \text { W } 518\end{array}$ & OSA & $\begin{array}{l}\text { Bronze Age } 4000- \\
3900 \text { bp }\end{array}$ & Total & - & MD-JC & 1993 \\
\hline 131 & $\begin{array}{l}\text { Camp Vermell } \\
\text { (Andorra) }\end{array}$ & $\begin{array}{l}42^{\circ} 27^{\prime} 51^{\prime \prime} \mathrm{N} 1^{\circ} 29^{\prime} \\
30^{\prime \prime} \mathrm{E} 940\end{array}$ & OSA & $\mathrm{XI}$-XII centuries & Partial & Yll et al. $2007 \mathrm{c}$ & $\begin{array}{l}\text { RY-FB- } \\
\text { IE }\end{array}$ & 2007 \\
\hline 132 & Can Olivé (Barcelona) & $\begin{array}{l}41^{\circ} 28^{\prime} 50^{\prime \prime} \mathrm{N} 2^{\circ} 08^{\prime} \\
06^{\prime \prime} \mathrm{E} 118\end{array}$ & OSA & Late Iron Age & Partial & Burjachs $\underline{1988 \mathrm{~b}}$ & FB & 1986 \\
\hline 133 & Canaleja I (Cáceres) & $\begin{array}{l}39^{\circ} 44^{\prime} 30^{\prime \prime} \mathrm{N} 5^{\circ} 42^{\prime} \\
1^{\prime \prime} \mathrm{W} 410\end{array}$ & OSA & $\begin{array}{l}\text { Chalcolithic 5000- } \\
4300 \text { bp }\end{array}$ & Partial & $\begin{array}{l}\text { López-Sáez and } \\
\text { López-Merino } 2007\end{array}$ & JLS & 2006 \\
\hline 134 & Cáparra (Badajoz) & $\begin{array}{l}38^{\circ} 57^{\prime} 14^{\prime \prime} \mathrm{N} 6^{\circ} 19^{\prime} \\
35^{\prime \prime} \mathrm{W} 278\end{array}$ & OSA & Roman & Total & Castillo et al. 1994 & PLG & 2002 \\
\hline 135 & Carvalhal (Portugal) & $\begin{array}{l}41^{\circ} 7^{\prime} 58^{\prime \prime} N 8^{\circ} 4^{\prime} 57^{\prime \prime} \\
\text { WV } 380\end{array}$ & OSA & Holocene & Total & Cruz 1991 & JLS & 2001 \\
\hline 136 & $\begin{array}{l}\text { Casa Montero } \\
\text { (Madrid) }\end{array}$ & $\begin{array}{l}40^{\circ} 29^{\prime} 37^{\prime \prime} \mathrm{N}^{\circ} 41^{\prime} \\
21^{\prime \prime} \text { W } 137732\end{array}$ & OSA & Early Neolithic & Total & Consuegra et al. 2004 & ЛLS & 1996 \\
\hline 137 & Castilmontain (Soria) & $\begin{array}{l}41^{\circ} 11^{\prime} 37^{\prime \prime} \mathrm{N} 22^{\circ} 18^{\prime} \\
51^{\prime \prime} \text { W } 875\end{array}$ & OSA & Celtiberic & Total & Arlegui 1992 & PLG & 1996 \\
\hline 138 & Castro de Vigo (Lugo) & $\begin{array}{l}43^{\circ} 12^{\prime} 17^{\prime \prime} \mathrm{N}^{\circ} 24^{\prime} \\
05^{\prime \prime} \mathrm{W} 147^{\prime}\end{array}$ & OSA & Late Holocene & Partial & $\begin{array}{l}\text { Aira-Rodriguez et al. } \\
1988\end{array}$ & MJA & - \\
\hline 139 & $\begin{array}{l}\text { Cerro San Vicente } \\
\text { (Salamanca) }\end{array}$ & $\begin{array}{l}40^{\circ} 57^{\prime} 41^{\prime \prime} \mathrm{N} 5^{\circ} 40^{\prime} \\
24^{\prime \prime} \mathrm{W} 780\end{array}$ & OSA & Early Iron Age & Partial & YIl et al. $2007 \mathrm{~b}$ & $\begin{array}{l}\text { RY-FB- } \\
\text { IE }\end{array}$ & 2007 \\
\hline 140 & Civiacas II (Huesca) & $\begin{array}{l}41^{\circ} 49^{\prime} 36^{\prime \prime} \mathrm{N}^{\circ} 9^{\prime} \\
24^{\prime \prime} \text { E } 280\end{array}$ & OSA & Bronze Age & Total & $\begin{array}{l}\text { González-Sampériz } \\
2001\end{array}$ & PGS & 1995 \\
\hline 141 & $\begin{array}{l}\text { El Castillejo de } \\
\text { Numancia (Soria) }\end{array}$ & $\begin{array}{l}41^{\circ} 48^{\prime} 35^{\prime \prime} \mathrm{N} 2^{\circ} 26^{\prime} \\
38^{\prime \prime} \mathrm{W} 1070\end{array}$ & OSA & Celtiberic & Partial & López-Garcia 1986 & PLG & 1996 \\
\hline 142 & E1 Molón (Valencia) & 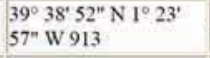 & OSA & Iberic & Total & - & JC & 1997 \\
\hline 143 & E Prado (Murcia) & $\begin{array}{l}38^{\circ} 27^{\prime} 19^{\prime \prime} \mathrm{N}^{\circ} 19^{\prime} \\
49^{\prime \prime} \mathrm{W} 470\end{array}$ & OSA & Bronze Age & Partial & López-Garcia 1988 & PLG & 1986 \\
\hline 144 & El Retamar (Cádiz) & $\begin{array}{l}36^{\circ} 31^{\prime} 44^{\prime \prime} \mathrm{N} 6^{\circ} 11^{\prime} \\
30^{\prime \prime} \mathrm{W} 10\end{array}$ & OSA & Early Neolithic & Total & Lozano et al, $\underline{1997}$ & PLG & 1996 \\
\hline 145 & El Vinyets (Tarragona) & $\begin{array}{l}41^{\circ} 11^{\prime} 14^{\prime \prime} \mathrm{N} 1^{\circ} 20^{\prime} \\
6^{\prime \prime} \mathrm{E} 82\end{array}$ & OSA & $\begin{array}{l}\text { Middle-Upper } \\
\text { Palaeolithic }\end{array}$ & Total & $\begin{array}{l}\text { Allué \& Renault- } \\
\text { Miskovsky } 1999\end{array}$ & EA & 1996 \\
\hline 146 & $\begin{array}{l}\text { Ereta del Pedregal } \\
\text { (Valencia) }\end{array}$ & $\begin{array}{l}39^{\circ} 6^{\prime} 5^{\prime \prime} \mathrm{N} 0^{\circ} 41^{\prime} 36^{\prime \prime} \\
\text { W } 225\end{array}$ & OSA & Neolithie-Bronze & Partial & Dupré et al. 1985 & MD & 1983 \\
\hline 147 & Estebanvela (Segovia) & $\begin{array}{l}41^{\circ} 25^{\prime} 9^{\prime \prime} \mathrm{N} 3^{\circ} 22^{\prime} \\
26^{\prime \prime} \mathrm{W} 982\end{array}$ & OSA & $11200-9900 \mathrm{bp}$ & Total & Cacho et al. $\underline{2003}$ & PLG & $2001-4$ \\
\hline 148 & Follente (Pontevedra) & $\begin{array}{l}42^{\circ} 36^{\circ} 19^{\prime \prime} \mathrm{N} 8^{\circ} 38^{\prime} \\
35^{\prime \prime} \mathrm{W} 24\end{array}$ & OSA & Holocene & Total & - & JLS & 2001 \\
\hline 149 & $\begin{array}{l}\text { Font del Ros } \\
\text { (Bareelona) }\end{array}$ & $\begin{array}{l}42^{\circ} 06^{\prime} 04^{\prime \prime} \mathrm{N} 1^{\circ} 50^{\prime} \\
47^{\prime \prime} \mathrm{E} 673\end{array}$ & OSA & Meso-Neolithic & Partial & Burjachs $\underline{1990}$ & FB & 1989 \\
\hline 150 & Fuentesaúco (Soria) & $\begin{array}{l}41^{\circ} 45^{\prime} 52^{\prime \prime} \mathrm{N} 2^{\circ} 20^{\prime} \\
11^{\prime \prime} \text { W } 814\end{array}$ & OSA & $\begin{array}{l}\text { Iron Age 2700- } \\
2200 \mathrm{bp}\end{array}$ & Partial & Mariscal 1994 & BM & - \\
\hline 151 & $\begin{array}{l}\text { Hoyo de La Mina } \\
\text { (Mălaga) }\end{array}$ & $\begin{array}{l}36^{\circ} 42^{\prime} 56^{\prime \prime} \mathrm{N} 4^{\circ} 16^{\prime} \\
31^{\prime \prime} \mathrm{W} 3\end{array}$ & OSA & Neolithic & Partial & $\begin{array}{l}\text { Cortés and } \\
\text { Sanchidrián } 1999\end{array}$ & PLG & 1999 \\
\hline 152 & $\begin{array}{l}\text { Huerta de los Cabreros } \\
\text { (Madrid) }\end{array}$ & $\begin{array}{l}40^{\circ} 1^{\prime} 43^{\prime \prime} \mathrm{N} 3^{\circ} 37^{\prime} 2^{\prime \prime} \\
\text { W } 500\end{array}$ & OSA & $\begin{array}{l}\text { Chalcolithic } 4150- \\
3950 \text { bp }\end{array}$ & Partial & Mariscal $\underline{1996}$ & BM & - \\
\hline 153 & $\begin{array}{l}\text { La Calzadilla } \\
\text { (Palencia) }\end{array}$ & $\begin{array}{l}41^{\circ} 24^{\prime} 40^{\prime \prime} \mathrm{N} 4^{\circ} 50^{\prime} \\
31^{\prime \prime} \mathrm{W} 900\end{array}$ & OSA & Bronze Age & Partial & - & ILS & 2005 \\
\hline 154 & $\begin{array}{l}\text { La Cativera } \\
\text { (Tarragona) }\end{array}$ & $\begin{array}{l}41^{\circ} 11^{\prime} 14^{\prime \prime} \mathrm{N} 1^{\circ} 20^{\prime} \\
6^{\prime \prime} \mathrm{E} 82\end{array}$ & OSA & Meso-Neolithic & Partial & $\begin{array}{l}\text { Allué \& Renault- } \\
\text { Miskovsky } 1999\end{array}$ & EA-JRM & - \\
\hline 155 & La Codera (Huesca) & $\begin{array}{l}41^{\circ} 43^{\prime} 39^{\prime \prime} \mathrm{N}^{\circ} 7^{\prime} \\
20^{\prime \prime} \mathrm{E} 219\end{array}$ & OSA & Bronze-Iron Age & Partial & Montón 1998 & PGS & 2004 \\
\hline 156 & La Fonollera (Gerona) & $\begin{array}{l}42^{\circ} 2^{\prime} 51^{\prime \prime} \mathrm{N}^{\circ} 6^{\circ} \\
55.73^{\prime \prime} \mathrm{E} 20\end{array}$ & OSA & Final Bronze & Total & Pons $\underline{1977}$ & FB & 1979 \\
\hline 157 & La Pijotilla (Badajoz) & $\begin{array}{l}38^{\circ} 43^{\prime} 37^{\prime \prime} \mathrm{N} 6^{\circ} 32^{\prime} \\
7^{\prime \prime} \mathrm{W} 260\end{array}$ & OSA & $\begin{array}{l}\text { Chalcolithic- } \\
\text { Bronze Age }\end{array}$ & Partial & Hurtado $\underline{2007}$ & JLS & 1998 \\
\hline 158 & La Prunera (Girona) & $\begin{array}{l}42^{\circ} 11^{\prime} 36^{\prime \prime} \mathrm{N} 2^{\circ} 30^{\prime} \\
43^{\prime \prime} \mathrm{E} 424\end{array}$ & OSA & Mesolithic & Partial & Burjachs $2000 \mathrm{~b}$ & FB & 1999 \\
\hline 159 & Las Monjas (Alicante) & $\begin{array}{l}38^{\circ} 20^{\prime} 41^{\prime \prime} \mathrm{N} 0^{\circ} 29^{\prime} \\
5^{\prime \prime} \mathrm{W} 5\end{array}$ & OSA & $\begin{array}{l}\text { Middle Age } \\
\text { (Muslim) }\end{array}$ & Total & - & IS & 2005 \\
\hline 160 & Los Millares (Almeria) & $\begin{array}{l}36^{\circ} 58^{\prime} 24^{\prime \prime} \mathrm{N} 2^{\circ} 31^{\prime} \\
32^{\prime \prime} \mathrm{W} 214^{\prime}\end{array}$ & OSA & Bronze Age & Total & Burjachs $1991 \mathrm{~b}$ & FB & 1990 \\
\hline 161 & Los Molares (Sevilla) & $\begin{array}{l}37^{\circ} 9^{\prime} 21^{\prime \prime} \mathrm{N} 5^{\circ} 43^{\prime} \\
11^{\prime \prime} \mathrm{W} 73\end{array}$ & OSA & Chalcolithic & Partial & $\begin{array}{l}\text { López-Garcia \& } \\
\text { López-Sáez } 1997\end{array}$ & PLG & 1990 \\
\hline 162 & Los Tholos (Badajoz) & $\begin{array}{l}38^{\circ} 41^{\prime} 5^{\prime \prime} N 6^{\circ} 24^{\prime} \\
12^{\prime \prime} \text { W } 340\end{array}$ & OSA & Chalcolithic & Total & - & PLG & 1998 \\
\hline 163 & Lulióbriga (Cantabria) & $\begin{array}{l}42^{\circ} 59^{\circ} 0^{\prime \prime} \mathrm{N} 4^{\circ} 6^{\prime} 56^{\prime \prime} \\
\text { W } 920\end{array}$ & OSA & Roman & Partial & Iglesias-Gil $\underline{1997}$ & - & - \\
\hline
\end{tabular}


Table 6. Cases of palynological sterility with open-air archaeological sites. Continuation.

\begin{tabular}{|c|c|c|c|c|c|c|c|c|}
\hline$\frac{\text { Site }}{\text { Number }}$ & Site & $\begin{array}{c}\text { Coordinates Altitude } \\
\text { m asl }\end{array}$ & Type & Age/Industry & Sterility & Reference & Analvst & $\begin{array}{l}\text { Lab } \\
\text { Year }\end{array}$ \\
\hline 164 & $\begin{array}{l}\text { Marroquies Bajos } \\
\text { (Jaén) }\end{array}$ & $\begin{array}{l}37^{\circ} 46^{\prime} 17^{\prime \prime} \mathrm{N} 3^{\circ} 47^{\prime} \\
17^{\prime \prime} \text { W } 548\end{array}$ & OSA & Chalcolithic- Iberic & Partial & Zafra et al. 1999 & PLG & 1997 \\
\hline 165 & $\begin{array}{l}\text { Mata el Casare } \\
\text { (Oviedo) }\end{array}$ & $\begin{array}{l}43^{\circ} 09^{\circ} 07^{\prime \prime} \mathrm{N} 5^{\circ} 56^{\prime} \\
31^{\prime \prime} \mathrm{W} 630\end{array}$ & OSA & Holocene & Partial & Dupré 1988 & MD & $1980 \mathrm{~s}$ \\
\hline 166 & Molinicos (Murcia) & $\begin{array}{l}38^{\circ} 12^{\prime} 27^{\prime \prime} \mathrm{N}^{\circ} 50^{\prime} \\
31^{\prime \prime} \mathrm{W} 460\end{array}$ & OSA & Chalcolithic & Partial & López-Garcia 1988 & PLG & 1986 \\
\hline 167 & $\begin{array}{l}\text { Monte Aguilar } \\
\text { (Navarra) }\end{array}$ & $\begin{array}{l}42^{\circ} 2^{\prime} 46^{\prime \prime} N 1^{\circ} 19^{\prime} \\
57^{\prime \prime} \text { W } 300\end{array}$ & OSA & Bronze Age & Partial & Iriarte $\underline{1992}$ & MI & - \\
\hline 168 & $\begin{array}{l}\text { Muntanya Assolada } \\
\text { (Valencia) }\end{array}$ & $\begin{array}{l}39^{\circ} 9^{\prime} 12^{\prime \prime} \mathrm{N}^{\circ} 0^{\circ} 22^{\prime} \\
52^{\prime \prime} \mathrm{W} 227\end{array}$ & OSA & Bronze Age & Total & Marti 1983 & MD & 1983 \\
\hline 169 & $\begin{array}{l}\text { Necrópolis del } \\
\text { Mercadillo (Cáceres) }\end{array}$ & $\begin{array}{l}39^{\circ} 20^{\prime} 46^{\prime \prime} \mathrm{N} 6^{\circ} 4^{\prime} \\
19^{\prime \prime} \mathrm{W} 420\end{array}$ & OSA & Iron Age & Partial & $\begin{array}{l}\text { Hernández \& Galản } \\
1992\end{array}$ & PLG & 1999 \\
\hline 170 & $\begin{array}{l}\text { Pedra Moura (La } \\
\text { Coruña) }\end{array}$ & $\begin{array}{l}43^{\circ} 09^{\prime} 09^{\prime \prime} \mathrm{N}^{\circ} 36^{\prime} \\
01^{\prime \prime} \mathrm{W} 1\end{array}$ & OSA & Late Holocene & Partial & $\begin{array}{l}\text { Aira-Rodriguez et al. } \\
1988\end{array}$ & MJA & $\cdot$ \\
\hline 171 & Piedrafita (Oviedo) & $\begin{array}{l}43^{\circ} 26^{\prime} 37^{\prime \prime} \mathrm{N} 5^{\circ} 59^{\prime} \\
51^{\prime \prime} \text { W } 400\end{array}$ & OSA & Holocene & Partial & Dupré 1988 & MD & $1980 \mathrm{~s}$ \\
\hline 172 & $\begin{array}{l}\text { Puente Largo del } \\
\text { Jarama (Madrid) }\end{array}$ & $\begin{array}{l}40^{\circ} 5^{\prime} 8^{\prime \prime} \mathrm{N} 3^{\circ} 36^{\circ} 16^{\prime \prime} \\
\text { W } 490\end{array}$ & OSA & Iberic 2650 bp & Partial & Mariscal $\underline{1996}$ & BM & - \\
\hline 173 & $\begin{array}{l}\text { Puntal dels Llops } \\
\text { (Valencia) }\end{array}$ & $\begin{array}{l}39^{\circ} 42^{\prime} 7^{\prime \prime} \mathrm{N} 0^{\circ} 32^{\prime} \\
31^{\prime \prime} \mathrm{W} 370\end{array}$ & OSA & Iberic & Total & Bonet et al. 1981 & MD RM & 1988 \\
\hline 174 & $\begin{array}{l}\text { San Bernardo } \\
\text { (Valladolid) }\end{array}$ & $\begin{array}{l}41^{\circ} 37^{\prime} 53^{\prime \prime} \mathrm{N}^{\circ} 15^{\prime} \\
43^{\prime \prime} \mathrm{W} 730\end{array}$ & OSA & Final Bronze & Total & - & IS & 2005 \\
\hline 175 & San Blas (Badajoz) & $\begin{array}{l}38^{\circ} 30^{\prime} 44^{\prime \prime} \mathrm{N} 7^{\circ} 16^{\prime} \\
52^{\prime \prime} \mathrm{W} 190\end{array}$ & OSA & $\begin{array}{l}\text { Chalcolithic } 5000- \\
4500 \text { bp }\end{array}$ & Total & Hurtado $\underline{2004}$ & IS & 2004 \\
\hline 176 & San José (Badajoz) & $\begin{array}{l}38^{\circ} 16^{\circ} 2^{\prime \prime} \mathrm{N} 6^{\circ} 28^{\prime} \\
27^{\prime \prime} \text { W } 495\end{array}$ & OSA & Bronze Age & Total & - & PLG & 2004 \\
\hline 177 & $\begin{array}{l}\text { San Martin de La Vega } \\
\text { (Madrid) }\end{array}$ & $\begin{array}{l}40^{\circ} 12^{\prime} 28^{\prime \prime} \mathrm{N} 3^{\circ} 34^{\prime} \\
6^{\prime \prime} \mathrm{W} 510\end{array}$ & OSA & Visigothic & Partial & López-Garcia $\underline{1983}$ & PLG & 1999 \\
\hline 178 & $\begin{array}{l}\text { San Miguel Atxa } \\
\text { (Álava) }\end{array}$ & $\begin{array}{l}42^{\circ} 51^{\prime} 54^{\prime \prime} \mathrm{N} 2^{\circ} 42^{\prime} \\
29^{\prime \prime} \text { W } 500\end{array}$ & OSA & Iron Age & Partial & Iriarte 1994 & MI & - \\
\hline 179 & Sevilleja (Jaén) & $\begin{array}{l}38^{\circ} 2^{\prime} 3^{\prime \prime} \mathrm{N} 3^{\circ} 51^{\prime} 44^{\prime \prime} \\
\text { W } 276\end{array}$ & OSA & $4800-800 \mathrm{bp}$ & Partial & Contreras et al, 1985 & $\pi \mathrm{S}$ & 1996 \\
\hline 180 & $\begin{array}{l}\text { Sitges de la UAB } \\
\text { (Barcelona) }\end{array}$ & $\begin{array}{l}41^{\circ} 29^{\prime} 59^{\prime \prime} \mathrm{N} 2^{\circ} 06^{\prime} \\
47^{\prime \prime} \text { E } 127\end{array}$ & OSA & Iron Age & Partial & $\begin{array}{l}\text { Burjachs 1988b: in } \\
\text { press }\end{array}$ & FB & 1986 \\
\hline 181 & $\begin{array}{l}\text { Teatro Romano } \\
\text { (Murcia) }\end{array}$ & $\begin{array}{l}37^{\circ} 35^{\prime} 58^{\prime \prime} \mathrm{N}^{\circ} 59^{\prime} \\
2^{\prime \prime} \mathrm{W} 15\end{array}$ & OSA & Roman 5-1 be & Total & Ramallo et al. 2004 & MM & 1999 \\
\hline 182 & Torre Roja (Alicante) & $\begin{array}{l}38^{\circ} 20^{\prime} 41^{\prime \prime} \mathrm{N} 0^{\circ} 29^{\circ} \\
5^{\prime \prime} \mathrm{W} 5\end{array}$ & OSA & Late Holocene & Total & - & JC-SF & 2006 \\
\hline 183 & $\begin{array}{l}\text { Tozal de Andrés } \\
\text { (Huesca) }\end{array}$ & $\begin{array}{l}41^{\circ} 57^{\prime} 26^{\prime \prime} \mathrm{N}^{\circ} 3^{\prime} \\
28^{\prime \prime} \mathrm{E} 380\end{array}$ & OSA & Iberic-Roman & Partial & $\begin{array}{l}\text { Gonzâlez-Sampériz \& } \\
\text { Sopena } 2002\end{array}$ & PGS & 1995 \\
\hline 184 & $\begin{array}{l}\text { Turó de la Bateria } \\
\text { (Girona) }\end{array}$ & $\begin{array}{l}41^{\circ} 59^{\circ} 40^{\prime \prime} \mathrm{N} 2^{\circ} 49^{\prime} \\
1^{\prime \prime} \mathrm{E} 77\end{array}$ & OSA & Upper Pleistocene & Partial & Yll et al. $2008 \mathrm{~b}$ & $\begin{array}{l}\text { RY-FB- } \\
\text { IE }\end{array}$ & 2007 \\
\hline 185 & Vale Cerva (Portugal) & $\begin{array}{l}41^{\circ} 7^{\prime} 7^{\prime \prime} \times 7^{\circ} 6^{\prime} 17^{\prime \prime} \\
\text { W } 380\end{array}$ & OSA & Holocene & Total & - & IS & 2005 \\
\hline 186 & $\begin{array}{l}\text { Vallparadis } \\
\text { (Barcelona) }\end{array}$ & $\begin{array}{l}41^{\circ} 33^{\prime} 47^{\prime \prime} \mathrm{N} 2^{\circ} 01^{\prime} \\
09^{\prime \prime} \mathrm{E} 330\end{array}$ & OSA & $\begin{array}{l}\text { Lower-Lupper } \\
\text { Pleistocene }\end{array}$ & Partial & Yll et al. 2007a & $\begin{array}{l}\text { RY-IE- } \\
\text { FB }\end{array}$ & 2007 \\
\hline 187 & $\begin{array}{l}\text { Venta Quemada I } \\
\text { (Toledo) }\end{array}$ & $\begin{array}{l}39^{\circ} 55^{\prime} 33^{\prime \prime} \mathrm{N} 3^{\circ} 58^{\prime} \\
59^{\prime \prime} \text { W } 593\end{array}$ & OSA & Bronze Age & Total & - & PLG & 1997 \\
\hline 188 & $\begin{array}{l}\text { Villasviejas de Tamuja } \\
\text { (Cáceres) }\end{array}$ & 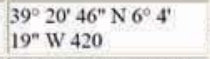 & OSA & $\begin{array}{l}\text { Late Iron Age } 2400 \\
\text { bp }\end{array}$ & Partial & Hemández et al. 1989 & PLG & 1999 \\
\hline 189 & Almonacid (Zaragoza) & $\begin{array}{l}41^{\circ} 16^{\prime} 23^{\prime \prime} \mathrm{N} 0^{\circ} 47^{\prime} \\
6^{\prime \prime} \mathrm{W} 500\end{array}$ & OSP T & Roman & Total & Pueyo et al. 2006 & PGS & 2002 \\
\hline 190 & $\begin{array}{l}\text { Cal Guardiola } \\
\text { (Barcelona) }\end{array}$ & $\begin{array}{l}41^{\circ} 34^{\prime} 06^{\prime \prime} \mathrm{N} 2^{\circ} 00^{\prime} \\
40^{\prime \prime} \mathrm{E} 310\end{array}$ & OSP T & $\begin{array}{l}\text { Middle-Lower } \\
\text { Pleistocene }\end{array}$ & Partial & Postigo er al. 2007 & FB & 1998 \\
\hline 191 & $\begin{array}{l}\text { Cuesta de la Bajada } \\
\text { (Teruel) }\end{array}$ & 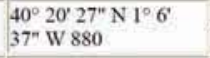 & OSP T & Middle Pleistocene & Partial & $\begin{array}{l}\text { Pérez-González et al. } \\
2000\end{array}$ & MD & $1990 \mathrm{~s}$ \\
\hline 192 & Matarraña (Teruel) & $\begin{array}{l}40^{\circ} 50^{\circ} 1^{\prime \prime} \mathrm{N} 0^{\circ} 10^{\circ} \\
47^{\prime \prime} \mathrm{E} 560\end{array}$ & $\begin{array}{l}\text { OSP } \\
\text { TR }\end{array}$ & $100-120 \mathrm{ka}$ & Total & Martinez-Tudela 1986 & JC & 1991 \\
\hline 193 & Quibas (Murcia) & 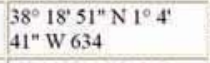 & $\begin{array}{l}\text { OSP } \\
\text { IR }\end{array}$ & 1.3-1 Ma & Total & Montoya et al. 2001 & JC & 2004 \\
\hline 194 & San Quirce (Palencia) & $\begin{array}{l}42^{\circ} 0^{\prime} 43^{\prime \prime} \mathrm{N} 4^{\circ} 3 \mathrm{I}^{\prime} \\
58^{\prime \prime} \mathrm{W} 738\end{array}$ & OSP T & Middle Pleistocene & Total & Arnáiz 1990 & MGA & 1991 \\
\hline 195 & $\begin{array}{l}\text { Guadalquivir } \\
\text { (Córdoba) }\end{array}$ & $\begin{array}{l}37^{\circ} 49^{\prime} 53^{\prime \prime} \mathrm{N} 5^{\circ} 14^{\prime} \\
22^{\prime \prime} \mathrm{W} 200\end{array}$ & TR & - & Total & 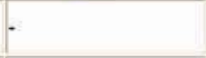 & SF-JC & 2006 \\
\hline 196 & $\begin{array}{l}\text { Tajo de Ronda } \\
\text { (Málaga) }\end{array}$ & $\begin{array}{l}36^{\circ} 44^{\prime} 30^{\prime \prime} \mathrm{N} 5^{\circ} 9^{\prime} \\
59^{\prime \prime} \mathrm{W} 700\end{array}$ & TR & - & Total & $*$ & SF-JC & 2007 \\
\hline 197 & $\begin{array}{l}\text { Islas Columbretes } \\
\text { (Castellón) }\end{array}$ & $\begin{array}{l}39^{\circ} 53^{\prime} 39^{\prime \prime} \mathrm{N} 0^{\circ} 41^{\prime} \\
11^{\prime \prime} \mathrm{E} 45\end{array}$ & PL & - & Partial & $\begin{array}{l}\text { Expósito \& Burjachs } \\
2007\end{array}$ & IE-FB & 2006 \\
\hline 198 & Pedro Bemardo (Ávila) & $\begin{array}{l}40^{\circ} 15^{\prime} 4^{\prime \prime} \mathrm{N}^{\circ} 54^{\prime} \\
20^{\prime \prime} \mathrm{W} 1095\end{array}$ & PL & Holocene & Partial & - & JS & 2004 \\
\hline 199 & Urda (Toledo) & $\begin{array}{l}39^{\circ} 24^{\prime} 42 \mathrm{~N} 3^{\circ} 42^{\prime} 57^{\prime \prime} \\
\text { W } 770\end{array}$ & PL & Holocene & Total & - & JS & 2006 \\
\hline 200 & Arzobispo (Toledo) & $\begin{array}{l}39^{\circ} 47^{\prime} 55^{\prime \prime} \mathrm{N} 5^{\circ} 11^{\prime} \\
23^{\prime \prime} \mathrm{W} 320\end{array}$ & T & - & Partial & $\begin{array}{l}\text { Martin-Arroyo et al. } \\
1996 \mathrm{a}\end{array}$ & TMA & - \\
\hline 201 & Valdelobos (Toledo) & $\begin{array}{l}39^{\circ} 5 \mathrm{I}^{\prime} 07^{\prime \prime} \mathrm{N} 4^{\circ} 0 \mathrm{I}^{\prime} \\
04^{\prime \prime} \mathrm{W} 500\end{array}$ & T & Pleistocene & Partial & $\begin{array}{l}\text { Martin-Arroyo et al. } \\
1996 \mathrm{~b}\end{array}$ & TMA & - \\
\hline 202 & Alós (Lleida) & $\begin{array}{l}41^{\circ} 54^{\prime} 42^{\prime \prime} \mathrm{N} 0^{\circ} 57^{\prime} \\
42^{\prime \prime} \text { E } 295\end{array}$ & $\begin{array}{l}\text { SD } \\
\text { TR }\end{array}$ & - & Total & - & PGS & 2004 \\
\hline
\end{tabular}


Table 6. Cases of palynological sterility with open-air archaeological sites. Continuation.

\begin{tabular}{|c|c|c|c|c|c|c|c|c|}
\hline $\begin{array}{c}\text { Site } \\
\text { Number }\end{array}$ & Site & $\begin{array}{c}\text { Coordinates Altitude } \\
\text { m asl }\end{array}$ & Type & Age/Industry & Sterility & Reference & Analyst & $\begin{array}{l}\text { Lab } \\
\text { Year }\end{array}$ \\
\hline 203 & $\begin{array}{l}\text { Barranco Hondo } \\
\text { (Teruel) }\end{array}$ & $\begin{array}{l}40^{\circ} 27^{\prime} 31^{\prime \prime} \mathrm{N} 0^{\circ} 48^{\prime} \\
56^{\prime \prime} \mathrm{W} 1400\end{array}$ & SD & - & Total & - & PGS & 2002 \\
\hline 204 & Las Lenas (Zaragoza) & $\begin{array}{l}41^{\circ} 32^{\prime \prime} \mathrm{N} 0^{\circ} 59^{\prime} 52^{\prime \prime} \\
\text { W } 440\end{array}$ & SD & Late Holocene & Total & - & PGS & 2003 \\
\hline 205 & Toiriz (Pontevedra) & $\begin{array}{l}42^{\circ} 47^{\prime} 49^{\prime \prime} \mathrm{N} 8^{\circ} 7^{\prime} \\
49^{\prime \prime} \mathrm{W} 400\end{array}$ & SD & Holocene & Partial & van Mourik $\underline{1985}$ & VMO & - \\
\hline 206 & Valmadrid (Zaragoza) & $\begin{array}{l}41^{\circ} 26^{\prime} 36^{\prime \prime} \mathrm{N}^{\circ} 53^{\prime} \\
4^{\prime \prime} \text { W } 580\end{array}$ & SD & $>18000 \mathrm{bp}$ & Partial & $\begin{array}{l}\text { González-Sampériz et } \\
\text { al. } 2005\end{array}$ & PGS & 2000 \\
\hline 207 & Tramacastilla (Huesca) & $\begin{array}{l}42^{\circ} 42^{\prime} 51^{\prime \prime} \mathrm{N}^{\circ} 18^{\prime} \\
59^{\prime \prime} \mathrm{W} 1732\end{array}$ & $\mathrm{MD}$ & - & Total & $\begin{array}{l}\text { González-Sampériz } \\
\text { 2004a }\end{array}$ & PGS & 1999 \\
\hline
\end{tabular}

1991a), La Pijotilla, Canaleja I (López-García and López-Sáez 1994a; 1994b), Buzanca I and Huerta de los Cabreros (Mariscal 1996), the Bronze Age of Monte Aguilar (Iriarte 1992), San Blas, San José, Venta Quemada I, Sevillejas, San Bernardo, La Calzadilla, El Prado (López-García 1991) and Cabezo Redondo (Fumanal et al. 1996), the Iron Age/Iberic sites of El Molón, San Miguel de Atxa (Iriarte 1994), Villasviejas de Tamuja, El Castillejo, Puente Largo del Jarama (Muñoz 2000), Castilmontán, Fuente Saúco (Mariscal 1994), Molinicos (López-García 1991) and Castro Follente (Table 6). Sandy sediments also parallel the lack of pollen in the open-air, historical sites of Castro de Vigo and Pedra Moura in Galicia (Aira-Rodríguez et al. 1988), Teatro Romano of Cartagena and Las Monjas and Torrerroja in Alicante. In other cases, sterility is in tandem with gypsum, as in the Neolithic site of Casa Montero, the Bronze Age site of San Bernardo, and the Visigothic site of San Martín de la Vega (López-García 1983).

Sands and red clays are associated with sterile palaeosols in central Spain: Urda in Toledo and Pedro Bernardo in Ávila (Table 6). In wetter climates, old soil horizons associated with settlement sites can, however, contain a lot of pollen and spores (van Geel et al. 1983; 2003) which are probably locked up in some form of humic complex (Dimbleby 1985). Slope deposits of Barranco Hondo, Las Lenas, Valmadrid, and the Portuguese Vale da Cerva at Guarda still show partial sterility and evidence of contamination by recent pollen (González-Sampériz et al. 2003a; González-Sampériz 2004a; Valero-Garcés et al. 2004) (Table 6).

Palaeontological sites can be also associated with doline infills, and fluvial and lakeshore terraces. Polliniferous layers sometimes result from areas that became buried in a general waterlogged phase, such as in the famous hominid site of Florisbad in South Africa (Scott and Nyakale 2002). Similarly, in all the palynological trials in Cal Guardiola (Tarrasa, Barcelona), the darker, more organic layers showed palynomorphs and plant macroremains, including timber (Postigo et al. 2007). The remaining layer suffered from oxidation and so lacked pollen (Burjachs 2000a; Peregrina 2003). Other sites were found to be fully sterile, like the mid-Pleistocene San Quince del Río Pisuerga and the early Pleistocene palaeontologically rich doline infilling of Incarcal (Girona) (Villalta and Vicente 1972; Galobart et al. 1990; Suc 1980; Geurts 1977;

\section{9; Leroy 1990) (Table 4).}

Exposed tufas and travertines of Alós (Lérida), the margins of the Guadalquivir River in Córdoba, Tajo de Ronda (Málaga), the Eemian from Río Matarraña (Beceite, Teruel), and the Lower Pleistocene of Sierra de Quíbas (Murcia) were barren of pollen, despite the presence of preserved macroremains in abundance suitable for detailed palaeobotanical studies (Martínez-Tudela 1986) or palaeontological ones (Montoya et al. 2001) (Table 6). Travertines, like breccias, can be polliniferous (Weinstein-Evron 1987; Vermoere et al. 1999). When dealing with these deposits, the possibility of contamination by recent or sub-recent pollen has to be kept in mind, as in Sterkfontein and other southern African hominin-bearing sites (Carrión and Scott 1999).

\subsection{Coprolites}

Dung accumulations, which occur in archaeological and palaeontological sites in caves or under rockshelters, may represent relatively unbiased pollen traps. However, fossil dung deposits are under-represented in the literature of pollen analysis. Carrión (2002b) demonstrated that pollen spectra from biogenic materials of animal origin were the best analogues of local and regional vegetation in the most arid areas of southeastern Iberia, and still showed the best analytical potential in terms of pollen concentration and taxon diversity. Dung pollen samples are sometimes not influenced by dietary preferences and offer a great potential for palynology, as is shown with bird guano (Horrocks et al. 2008), Procavia and Petromus middens (Scott and Cooremans 1992; Carrión et al. 1999b; Gil-Romera et al. 2007), middens of packrat (Neotoma) and other rodents (Davis and Anderson 1987; Betancourt 2004), cow dung (Carrión et al. 2000b), coprolites of extinct caprids (Alcover et al. 1999), hyena coprolites (Scott 1987; Scott et al. 2003; González-Sampériz et al. 2003b; Yll et al. 2006), bat guano (Carrión et al. 2006b; Leroy and Simms 2006), sheep/goat and human coprolites from old farms (Hunt et al. 2001), and canid coprolites (González-Sampériz 2004a). Among these, hyena coprolites have been the most tested in the Iberian Quaternary (Fernández-Rodríguez et al. 1995; Carrión et al. 2001a; 2007a; González-Sampériz et al. 2003b).

The case studies considered here are pertinent to coprolites of three genera of hyaenids, namely Chasmaporthetes, Pachy- 
Table 7. Quaternary sites of Iberia with reported sterility in coprolite pollen samples.

\begin{tabular}{|c|c|c|c|c|c|c|c|c|}
\hline$\frac{\text { Site }}{\text { Number }}$ & Site & $\begin{array}{l}\text { Coordinates } \\
\text { Altitude m asl }\end{array}$ & Agent & Age & Sterility & Reference & Analvst & $\begin{array}{l}\text { Lab } \\
\text { Year }\end{array}$ \\
\hline 208 & $\begin{array}{l}\text { Abric Romaní } \\
\text { (Barcelona) }\end{array}$ & $\begin{array}{l}41^{\circ} 32^{\prime} 2^{\prime \prime} \mathrm{N}^{\circ} 41^{\prime} \\
25^{\prime \prime} \mathrm{E} 330\end{array}$ & Cf. Crocuta & $70-40 \mathrm{ka}$ & Total & Burjachs 2002 & FB & $\begin{array}{l}1998- \\
2003\end{array}$ \\
\hline 209 & $\begin{array}{l}\text { Cal Guardiola } \\
\text { (Barcelona) }\end{array}$ & $\begin{array}{l}41^{\circ} 33^{\prime} 39^{\prime \prime} \mathrm{N} 2^{\circ} 1^{\prime} \\
3^{\prime \prime} \text { E } 271^{\prime}\end{array}$ & Unknown & $\begin{array}{l}\text { Lower-Middle } \\
\text { Pleistocene }\end{array}$ & Partial & $\begin{array}{l}\text { Peregrina 2003: } \\
\text { Postigo et al. } 2007\end{array}$ & FB & 1998 \\
\hline 210 & $\begin{array}{l}\text { Cueva Victoria } \\
\text { (Murcia) }\end{array}$ & $\begin{array}{l}37^{\circ} 37^{\prime} 56^{\prime \prime} \mathrm{N} 0^{\circ} 49^{\prime} \\
16^{\prime \prime} \mathrm{W} 60\end{array}$ & Pachycrocuta & $\begin{array}{l}\text { Lower } \\
\text { Pleistocene }\end{array}$ & Total & Gibert et al. 1995 & JC & 2003 \\
\hline 211 & Fonelas (Granada) & $\begin{array}{l}37^{\circ} 24^{\prime} 45^{\prime \prime} \text { N } 3^{\circ} 12^{\prime} \\
10^{\prime \prime} \mathrm{W} 800\end{array}$ & Chasmaporthetes & Plio-Pleistocene & Total & Arribas et al. $\underline{2004 \mathrm{~b}}$ & JC & $2000-4$ \\
\hline 212 & $\begin{array}{l}\text { Gorham's } \\
\text { (Gibraltar) }\end{array}$ & $\begin{array}{l}36^{\circ} 7^{\prime} 16^{\prime \prime} \mathrm{N} 5^{\circ} 20^{\prime} \\
32^{\prime \prime} \text { W } 5\end{array}$ & Crocuta & $46-11 \mathrm{ka}$ & Partial & Carrión et al. 2008 & JC & 2003-5 \\
\hline 213 & Grajo (Córdoba) & $\begin{array}{l}37^{\circ} 26^{\prime} 14^{\prime \prime} \mathrm{N} 4^{\circ} 11^{\prime} \\
24^{\prime \prime} \mathrm{W} 625\end{array}$ & Crocuta & $\begin{array}{l}\text { Mid-Upper } \\
\text { Pleistocene }\end{array}$ & Total & $\begin{array}{l}\text { Riquelme et at. } \\
2004\end{array}$ & JC-SF & 2001 \\
\hline 214 & $\begin{array}{l}\text { Las Ventanas } \\
\text { (Granada) }\end{array}$ & $\begin{array}{l}37^{\circ} 26^{\prime} 25^{\prime \prime} \mathrm{N}^{\circ} 26^{\prime} \\
1^{\prime \prime} \mathrm{W} 1056\end{array}$ & Crocuta & 12780 cal bp & Partial & Carrión et al.2001a & JC & 2000 \\
\hline 215 & Nerja (Málaga) & $\begin{array}{l}36^{\circ} 45^{\prime} 42^{\prime \prime} \mathrm{N} 3^{\circ} 52^{\prime} \\
6^{\prime \prime} \mathrm{W} 110\end{array}$ & Crocuta & $30-4 \mathrm{ka}$ & Total & Arribas et al. $2004 \mathrm{a}$ & JC & 2001 \\
\hline 216 & Oliveira (Portugal) & $\begin{array}{l}39^{\circ} 29^{\prime} 49^{\prime \prime} \mathrm{N} 8^{\circ} 36^{\prime} \\
59^{\prime \prime} \text { W } 89\end{array}$ & Crocuta & $\begin{array}{l}\text { Mousterian } \\
40400-31900 \text { bp }\end{array}$ & Partial & Zilhao $\underline{2001}$ & JC-SF & 2005 \\
\hline 217 & Orce (Granada) & $\begin{array}{l}37^{\circ} 43^{\prime} 17^{\prime \prime} \mathrm{N} 2^{\circ} 28^{\prime} \\
45^{\prime \prime} \mathrm{W} 940\end{array}$ & Pachycrocuta & $\begin{array}{l}\text { Lower } \\
\text { Pleistocene }\end{array}$ & Total & Gibert et al. 1988 & JC & 1999 \\
\hline 218 & $\begin{array}{l}\text { Torrejones } \\
\text { (Guadalajara) }\end{array}$ & $\begin{array}{l}41^{\circ} 0^{\prime} 41^{\prime \prime} \mathrm{N} 3^{\circ} 15^{\prime} \\
2^{\prime \prime} \mathrm{W} 1100\end{array}$ & Crocuta & $80-60 \mathrm{ka}$ & Partial & Carrión et al. 2007a & JC & $2005-6$ \\
\hline 219 & $\begin{array}{l}\text { Vallparadis } \\
\text { (Barcelona) }\end{array}$ & $\begin{array}{l}41^{\circ} 33^{\prime} 47^{\prime \prime} \mathrm{N} 2^{\circ} 01^{\prime} \\
09^{\prime \prime} \text { E } 330\end{array}$ & Crocuta & $\begin{array}{l}\text { Lower-Upper } \\
\text { Pleistocene }\end{array}$ & Total & YIl et al. 2008a & $\begin{array}{l}\text { RY-IE- } \\
\text { FB }\end{array}$ & 2007 \\
\hline 220 & $\begin{array}{l}\text { Venta Micena } \\
\text { (Granada) }\end{array}$ & $\begin{array}{l}37^{\circ} 43^{\prime} 58^{\prime \prime} \mathrm{N} 2^{\circ} 23^{\prime} \\
16^{\prime \prime} \mathrm{W} 942\end{array}$ & Pachycrocuta & $\begin{array}{l}\text { Lower } \\
\text { Pleistocene }\end{array}$ & Total & $\begin{array}{l}\text { Arribas \& } \\
\text { Palmqvist } 1998\end{array}$ & JC-SF & 2005 \\
\hline 221 & $\frac{\text { Villacastin }}{\text { (Segovia) }}$ & $\begin{array}{l}40^{\circ} 47^{\prime} 52^{\prime \prime} \mathrm{N} 4^{\circ} 22^{\prime} \\
20^{\prime \prime} \mathrm{W} 1123\end{array}$ & Crocuta & $150-120 \mathrm{ka}$ & Partial & Carrión et al. $2007 \mathrm{a}$ & JC-GR & $2005-6$ \\
\hline
\end{tabular}

crocuta and Crocuta (Table 7). Tens of light whitish coprolites, presumably produced by Chasmaporthetes lunensis, from the Fonelas sites (Guadix-Baza basin, Granada) were palynologically sterile. These coprolites were stuffed in lutites. Likewise, cases of full sterility come from a few Pachycrocuta brevirostris coprolites from the Lower Pleistocene of Cueva Victoria (Murcia), and Venta Micena-Orce (Granada) (Table 7) (Carrión et al. 2004c). Although there were items rich in pollen, a number of the analysed coprolites of Crocuta crocuta from Villacastín and Torrejones (Carrión et al. 2007a), Cueva de las Ventanas (Carrión et al. 2001a), Oliveira in Portugal (Zilhao 2001) and Gorham's Cave in Gibraltar (Carrión et al. 2008), were sterile. All Crocuta coprolite specimens were sterile in Abric Romaní, Barcelona (Allué et al. 1998; Burjachs 2002), while the rockshelter travertine was polliniferous (Burjachs and Julià 1994; 1996). Similarly, there was total sterility in the coprolites of Andalusian Cueva del Grajo and Cueva de Nerja. Potential for work with other hyena species exists because, for instance, Hyaena brunnea (brown hyena) has been identified in south-eastern Spain (Arribas et al. 2004b). Hyaena brunnea coprolites from the southern African sites of Equus Cave (Taung, southern Kalahari) and Oyster Bay (Cape region) were successfully treated for pollen (Scott 1987; Carrión et al. 2000c), although total pollen concentrations in the coprolites were lower than those sometimes observed in Crocuta (Scott et al. 2003).

\section{Mechanisms for the Destruction of Pollen}

Although this is not an article on pollen preservation and decay, reasonable speculation about the causes of sterility can be attempted on the basis of the above observations. Peats are mostly polliniferous, but sterile levels of mineral sediment may interrupt the peat sequence. This is the case in Los Monjes, Comella, Mozarrifar, and especially Navarrés (Table 3), with sandy sediment layers suggesting increased processes of erosion of the surroundings, and, in fact, a depositional context favourable for oxidation of pollen (Carrión and van Geel 1999).

Exploitation, drainage, salinisation, contamination and trenching diminish the analytical potential of peats. In Ruidera (Ciudad Real), a barrage tufa wetland (García del Cura et al. 2000), peat layers overlying carbonated marls were almost completely sterile (Table 3 ). It is plausible that a reduction in excessive groundwater altered the polliniferous possibilities of the sediment by changing the original redox conditions (Dorado-Valiño et al. 2002). In addition, the lack of less mineralised groundwater inputs has caused an increase in salinisation. In other cases, like Villaverde (Carrión et al. 2001b) (Table 3), trenching a peat section resulted in aeration and break-up of the deposit structure, with subsequent oxidation of pollen (Havinga 1984). Trenching and peat extraction on areas of intact peat bog may in part have caused pronounced changes in the hydrological regime, which would in turn have influenced the vegetation and increased peat decomposition. At this point, it is quite possible that the drainage ditches irreversibly influenced the intact 
part of the bog. A systematic study of the hydrological regime in the Villaverde peat bog is needed to confirm this hypothesis. Eventually, the dams of the ditches should be blocked in order to prevent further desiccation of the area and to bring the hydrological regime more closely into line with the natural regime. Specifically, with tufas there must be some connection between pollen occurrence and depositional morphotypes (e.g. braided, barrage travertine, fluvial barrage, and marsh tufas), timing of inorganic deposition, and types of organically induced facies in travertine formation (Ford and Pedley 1996). No experimental studies have hitherto been devoted to this issue, to our knowledge.

It cannot be stressed enough that human activities may contribute to the irreversible loss of the potential of the few Iberian peats suitable for pollen analysis. The Padul peat bog (Pons and Reille 1988) and other wetlands from the Betic cordilleras, like Sierras de Baza, Filabres, even Sierra Nevada, have been greatly altered during recent decades despite the existence of initiatives for conservation (Casado and Montes 1995; Rodríguez-Sánchez 1998). At their current rate of spread, urban settlements will soon impede any possibility of studying littoral marshlands in Mediterranean Spain (Ortega et al. 2004). Old peat lands from Villena and Sax (Alicante), Mazarrón and Calblanque (Murcia), Cueto de Avellanosa (Cantabria), and Saldropo (País Vasco), among others, have now nearly vanished.

As recently as between 1956 and 1987, the area covered by peat in the Doñana National Park was dramatically reduced by almost 90\% (Sousa and García-Murillo 1999; Fernández-Zamudio et al. 2007. The opportunities for palynology have therefore become more limited. The most saline environments ('marisma') are difficult for pollen analyses. In the studies performed on the late Holocene Carrizosa, Cherri, Juncabalejo, Membrillo, and Vetalengua marshlands of Doñana (RodríguezRamírez et al. 1996), not only are there a number of palynological hiatuses, but also an extraordinary prevalence of marsh pollen (chenopods, sedges, Alismataceae) as well as thick layers where decomposing fungal activity predominates (Yáñez 2005). Overall in these wetlands, pollen-stratigraphical changes and, indeed, the potential of pollen analysis, are strongly dependent on changing sediment types as a result of geomorphological dynamics. In general, marine sedimentation events coincide with erosion, deposition of sands, destabilisation of the marisma, and palynological sterility (Yáñez et al. 2006). The stabilisation of the marisma coincides with colonisation by sedges and pollen deposition. Pollen concentration is low in evolved marisma phases, with long seasonal periods of dryness, and increased decomposing activity.

The situation with saline lacustrine systems is not simple. Failures with the endorheic lakes of La Mancha are worth scrutiny, where it is tempting to look at lithological features (Table 4). The sediments from Pétrola were dark grey to red clays, episodically interweaved with sands, peat, and carbonated crusts. Acequión was a brown marl with grey silts in the uppermost two metres. Ontalafia was light reddish sand with gravels and clays grading upwards to compacted silt. In spite of these differences, carbonates and, especially, signs of oxidation were observed throughout the three cores, and chlorides and sulphates (anhydrite and gypsum) very common in Pétrola, and sparse in the other two sites. Like pyrite in reductive environments, carbonates and sulphates can be frequent in lacustrine basins of semi-arid regions (Horowitz 1992). The same is true for salt pans, where crystal growth (lithification) in and around the pollen grains may be a cause of mechanical damage. Today, water conductivity is very high in the hypersaline Pétrola (16 $\mathrm{mS} \mathrm{cm} \mathrm{cm}^{-1}$ ), and relatively high in Ontalafia $\left(4.85 \mathrm{mS} \mathrm{cm}^{-1}\right)$ (Reed 1998), with abundance of magnesium sulphate in the former and sodium chlorides in the latter (Cirujano 1990). An unpublished sedimentary analysis (M.P. Fumanal) has pointed to long desiccation phases and stationary regimes in the three lakes, probably because of high summer evapotranspiration. Wetting and drying the pollen before burial are a major cause of alteration of the exines (Holloway 1989).

The Salineta lake in Bujaraloz (Zaragoza) adds episodic aeolian deflation (evaporation as a result of wind) to the seasonal character of the water body and saline nature of the sediment as likely factors of pollen decay and/or removal (Moreno et al. 2004; Valero-Garcés et al. 2004) (Table 4). Very similar are the nearby La Playa and Mediana de Aragón playa-lakes, where sterile layers are clearly associated with the highest concentrations of soluble salts (González-Sampériz et al. 2008). In Valsalada (Leciñena, Zaragoza), the absence of pollen parallels gypsum deposition and fluvial inputs (Sancho et al. 2007). In Laguna del Villardón in the playa-lake complex of Villafáfila (Zamora), a sandy silt core contained no pollen grains but several types of more resistant non-pollen palynomorphs (GómezFerreras et al. 1996). Laguna de Gallocanta (Teruel-Zaragoza), a temporary salt lake with discontinuous sedimentation, shows an alternation of sterile and polliniferous levels, although the latter show low pollen concentrations (Burjachs et al. 1996; Julià et al. 2000; Rodó et al. 2002). Today, both Salineta and Gallocanta exhibit high water conductivity of about $200 \mathrm{mS}$ $\mathrm{cm}^{-1}$ and $\mathrm{pH}$ between 8 and 9 (Reed 1998).

Salinity measurements cannot yet be used simply to signify a general trend of palynological sterility in salt lakes because, as in other arid regions of the world (Luly 1997; Davis 1998; Scott 1999 ), they have not always been entirely negative for palynologists. Sites like La Salineta, La Playa, and Mediana de Aragón (Table 4) have eventually been profitable, even considering hiatuses. Smaller saline systems including playa-lakes in north-eastern (Stevenson et al. 1991; Valero-Garcés et al. 2000a, b; González-Sampériz et al. 2008) and south-eastern Spain (Burjachs et al. 1997) have also produced satisfactory outcomes (Rodrigo et al. 2002). Pollen analysis of pure halite has provided good pollen spectra in the Dead Sea (Heim et al. 1997). An interesting case of success in Iberia is Lake Zóñar (Alonso 1998), where detailed palynological studies are being developed with ongoing projects (Valero-Garcés et al. 2006a; Martín-Puertas et al. 2008). Waters in Zóñar are certainly saline $\left(2.4 \mathrm{~g}^{-1}\right)$, alkaline ( $\mathrm{pH}$ between 7.1 y 8.4$)$ and dominated by 
$\left(\mathrm{Cl}^{-}\right)-\left(\mathrm{SO}_{4}^{2-}\right)$ and $\mathrm{Na}^{+}$(Valero-Garcés et al. 2006a), but a positive factor is most certainly the permanent character of the lake during most of the sequence. In sum, salt deposition is sometimes associated with desiccation and loss of pollen, but the halophilous character of a system should not discourage pollen analysis.

In general, non-saline lake sediments are favourable for pollen preservation, but problems will generally arise in very shallow systems that undergo seasonal periods of dryness and when intense inwash of soils from the surroundings exacerbates sediment dilution by soil that is usually pollen-barren (Table 4). Changes to non-lacustrine facies may still be conducive to sterility. Thus, a marine intrusion is often linked to oxidation both at the beginning and at the end of the phase. In the PegoOliva marshland, pollen-sterile intervals correspond with marine sediments and with peaks of detrital sulphates (Dupré et al. 1998b).

Palaeo-lakes occasionally may be sterile throughout their whole sequence or include sterile levels (Table 4). In sites like the Lower Pleistocene Mencal, Fonelas and others of the Orce complex (Zagales, Yeseras, Conejos, Champiñones), it is clear that erosion, fast sedimentation, re-sedimentation and water transport have not been favourable to the stabilisation of pollen assemblages. In north-eastern Spain, Mas Miquel, Bòbila Ordis, Incarcal, Tres Pins, and Pla de l'Estany show erosional, oxidised levels lacking pollen (Geurts $\underline{1977}$; Leroy 1990; 1997; 2008; Løvlie and Leroy 1995) and interruption of lacustrine sequences by soils, which are by nature often sterile.

The situation is no different with the so-called open-air archaeological and palaeontological sites, where post-depositional alteration and loss of pollen content is frequent (Table 6). High-energy environments in fluvial, aeolian and open-air contexts are normally to be avoided as oxidation and mechanical factors jointly act to destroy palynomorphs. Clearly for these prehistoric sites, we need experimental studies similar to those of Macphail et al. (2004), which deal with the relationships between pollen decay and soil micromorphology and microchemistry.

At first sight, sterility in caves and rockshelters (Table 5) is not surprising given the bad reputation of cave palynology. Sedimentary discontinuities (Campy and Chaline 1993), selective preservation, preferential transport, and contamination by percolating water and bioturbation (Coûteaux 1977; Turner and Hannon 1988) have often been claimed as causing negative results. Certainly, these sites have traditionally suffered from a dearth of experimental data capable of determining the effectiveness of cave pollen spectra in representing source vegetation. But the most worrying factor is not whether the pollen assemblages may or may not reflect the environments of the catchment areas, because we now know that they may do so (Coles et al. 1989; Burney and Burney 1993; Coles and Gilbertson 1994), especially in areas with an entomophilous-dominated flora (Navarro et al. 2002) and especially if several profiles are studied for the same cave (Carrión et al. 1999a). A more serious challenge arises from our current inability to identify characteristics and modes of post-depositional alteration. A high number of Asteraceae and Pteridophyta types can be indicative of this (Bottema and Woldring 1994), but only if coinciding with low pollen concentration and high counts of indeterminable pollen (Carrión 1992a; Sánchez-Goñi 1994). In either case, correlation with conventional pollen sequences demonstrates the usefulness of some cave records (Fernández et al. 2007). So, after taking due precautions, the usefulness of depending upon cave sediments in areas where conventional pollen-rich deposits are rare must not be overlooked.

As with peats, sandy layers and clastic strata in cavities usually involve loss of the pollen content (Dupré 1988; GonzálezSampériz 2004a). But caves are a special case. Most cave and rockshelter stratigraphies show sedimentary features indicative of complex depositional and post-depositional, physical and geochemical processes, several of which lead to alteration of biotic remains, including pollen grains and spores (Table 5). Burrowing, whether by insects, earthworms or rootlets, is a very negative influence. Problems linked to diagenesis appear critical. Red clay beds, associated with the alteration of ironbearing minerals, often result in sterility, such as in Bolomor (Fernández-Peris 2004), and Calaveres (Vives 1982; Dupré 1988), Cueva del Canuto at Sierra de Grazalema in Cádiz, and El Pendo in the Cantabrian region (Leroi-Gourhan 1980; López-García 1986; Sánchez Goñi 1991) (Table 5). But while reddish colour may suggest oxidation, we generally lack information about whether it took place before or after the incorporation of pollen. So doubt usually persists about the respective timing of pollen deposition and oxidation. In other words, a red colour can indicate erosion of previously red rock formations, not necessarily in situ oxidation. The same question arises with manganese oxides characteristic of some occupation layers within caves, as in Mousterian Carihuela Chamber III (Carrión et al. 1998). Here, as in Cueva de Chaves (Table 5), the sediments formed under the driest conditions were polliniferous, which substantiates the value of total aridity for biotic preservation and the negative effect of sediment moisture and frequent soil hydration-dehydration cycles (Davis 1990; Navarro et al. 2002). In Atapuerca, episodic washing and oxidation of microfossil assemblages dominate the post-depositional environment, resulting in an almost total absence of pollen and phytoliths (Vallverdú et al. 2001).

The case of Cova Beneito is notable because no observable difference was noted either texturally or structurally in the polliniferous sediments (Carrión and Munuera 1997) with respect to the sterile sediments (Carrión 1992a). Basically, most levels displayed an angular coarse fraction within a clayey-silt matrix. Measurements of $\mathrm{pH}$ showed relatively high values in all sediments, but their variation was insignificant, from 7.7 to 8.3. Pollen was relatively well preserved in samples with high $\mathrm{pH}$ values. There is hardly any doubt that the fact that the polliniferous profiles had been freshly exposed served to give better results than samples removed from sections left open on 
old excavations (Scott 1982; 1995).

Studies of modern pollen deposition suggest that cave morphology can be important for pollen analysis. So there should be a spatial patterning of sediments and pollen influx (Hunt and Rushworth 2005). For example, some caves show a fall-off in pollen concentration with increasing distance from the entrance (Burney and Burney 1993; Navarro et al. 2000; 2001). The Cueva de la Plata, a narrow, small-entranced, long cavity in coastal Murcia, showed lower pollen concentrations than the nearby Cueva de José, an isodiametric, wide-entranced cavity (Prieto and Carrión 1999; Navarro et al. 2000). The same situation was observed between Cueva del Moro I and II in Alicante (Navarro et al. 2000; 2001). However, the fact that a cave displays large chambers and wide entrances seems not to guarantee success with pollen analysis. In the cases of Chaves, El Salt, Cova Negra, Cendres, and Bolomor (Table 5), the successful profiles were located relatively close to the cave opening and some distance away from the cave walls (Fumanal 1986; Fernández-Peris 2004). In several of the caves for which modern pollen deposition was studied, wet sediment and parietal samples, as well as those samples taken from dripping areas, showed biased pollen spectra with low pollen concentration, high percentages of non-pollen microfossils such as fungal spores, and raised percentages and concentration values of Cichorioideae (Prieto and Carrión 1999; Navarro et al. 2001). Hence, degradation could occur in this context, and this could explain the aforementioned case of Cova Beneito. It is worth stressing that the two successful new sections studied (5C and 3B) were situated closer to the centre of the cavity (Carrión and Munuera 1997).

The cases of success with cave hearth levels are interesting (Table 5), as is the presence of pollen in burnt cow-dung (Carrión et al. 2000b) and bread samples (Williams-Dean 1978). When hearths are poorly compacted, it is difficult to disregard percolation from overlying strata. Hearths usually contain a mixture of ashes and windblown dust, forming a fine-grained, highly organic deposit. Most of the cave infill of Matutano Cave was composed of this type of material, making it very difficult to process for pollen extraction (Burjachs 1999). Supposedly, pollen grains should be burnt out by high-temperature fire, but it is also possible that they are resistant to low-temperature fire and trapped together with fine dust after burning until heat subsides (Horowitz 1992).

Cementation processes of any kind may also cause mechanical degradation of pollen grains (Table 5). However, stalagmitic units were extremely rich in pollen within Carihuela Cave (Carrión et al. 1998; Fernández et al. 2007), and there are several interesting case studies in the British Isles (McGarry and Caseldine 2004; Caseldine et al. 2007) and Africa (Burney et al. 1994), showing the enormous potential of speleopalynology including the distinct advantage over other pollen sources that they can be dated by high precision TIMS U-Th dating. Recently, Lartigot (2007) has provided a detailed account of the problems of palynology in cave speleothems from hominin- bearing caves in France and Italy, with low pollen concentration being one of the biggest challenges. In general, as with unconsolidated infills, it seems that entrance facies are more favourable for palynology (Fernández-Cortés et al. 2006). This could explain the total absence of pollen in the large speleothems studied from La Blanca (Murcia) and the gypsum speleothems of the Sorbas karst (Almería), both collected in the inner parts of deep karstic caverns. Certainly, other factors are involved, such as speleothem mineralogy, content of organic matter, and distance of the pollen sample from drip points, cave floor/ceiling, and flowstone limbs.

Clearly, cave palynology still needs much experimentally based work before we can predict successful contexts for pollen analysis. The available aforementioned studies indicate a great complexity in the taphonomy of pollen and spores. Both depositional and preservational features of the pollen spectra inside caves are uneven and clearly influenced by the cave morphology and sedimentary types. Stochastic and episodic forms of particle influx, such as transport by animals, periodic flooding and human activities, may also influence pollen deposition inside caves in proportions that are unique to each site. Caves in which the dominant type of pollen transfer from the external environment is airborne will often show a decrease in pollen deposition with increasing distance into the cave. Generally, in these cases, the highest concentrations of pollen and spores are observed in the cave entrance areas, and the lowest at the rear of the cave. Navarro et al. $(\underline{2000} ; \underline{2001})$ provide two basic recommendations for the pollen analysis of cave sediments. Firstly, that sampling is undertaken on the basis of a multipleprofile strategy, if possible not very close to parietal and rear areas and avoiding zones of actual moisture, or areas where old hydromorphic processes can be detected from sedimentological features. Secondly, it is of vital importance to use all the available information (pollen percentages, concentration, diversity and preservation) to establish a robust taphonomical model. This might facilitate the isolation of abnormal inputs, i.e. overrepresentation of some taxa, allowing a more reliable ecological interpretation of the data.

Preservation in coprolites has still to be understood; there may be factors such as digestive enzymes in addition to others mentioned so far. Why Crocuta and Hyaena coprolites have given pollen, while Pachycrocuta and Chasmaporthetes failed, remains puzzling (Table 7). Dietary variations seem unlikely since there is no crucial difference in hunting-scavenging behaviour between the four genera. Hyaena brunnea can certainly be more omnivorous than Crocuta, but most species are rather versatile in diet (Scott 1987). It is worth considering whether the Hyaena and Crocuta coprolites are polliniferous simply because the analysed sites are younger, and fossilisation processes in older samples of dung work against pollen preservation (Scott et al. 2003).

In sum, oxidation might be the main factor causing palynological sterility in the cases reported (Fig. 3). Hypothetically, oxidation occurs at different stages between the plant pollen- 


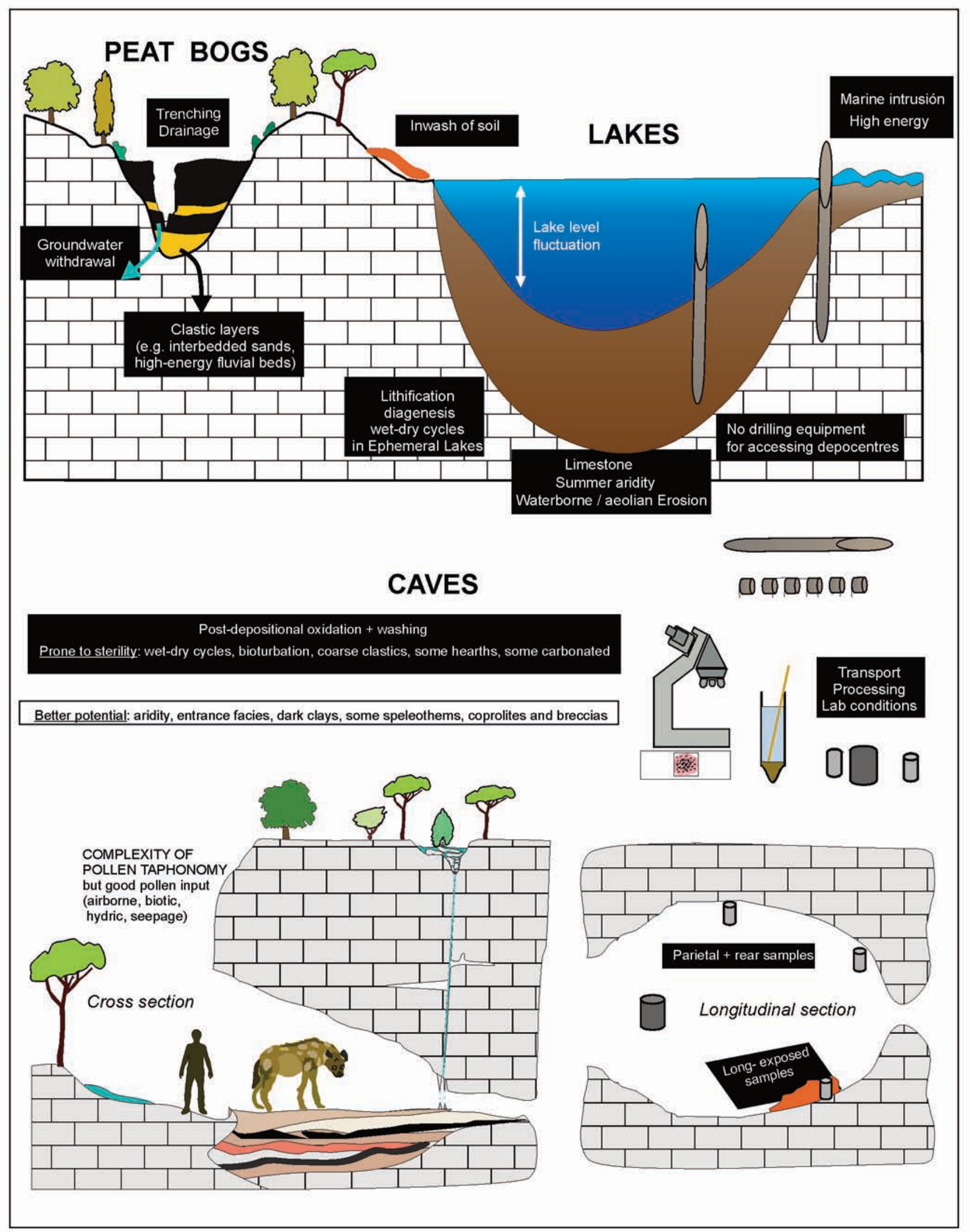

Figure 3. Scheme of the main causes of sterility in the Iberian Peninsula as applied to peat bogs, lakes and cave systems. 
producing organ and the microscope: (i) pre-depositional, e.g. soil inwash in lakes and peat bogs; (ii) syn-depositional, e.g. high-energy sediment; (iii) post-depositional, e.g. fluctuation of lake levels; and (iv) post-excavational, e.g. during field sampling, sample preparation, and on the microscope slide. Arguably, the number of wet-dry cycles (or oxidation-reduction cycles), the duration of the exposure to air, as well as the role of decomposing bacteria and fungi, are critical factors.

\section{Final Remarks}

Failed pollen analyses in the Iberian Peninsula are so numerous as to suggest that there may be something intrinsic to this region that is inimical to the preservation of palynomorphs. Is the huge mass of calcium carbonate represented by the Iberian Peninsula somehow related to palynological sterility? Is the prevailing aridity/summer drought a limiting factor? Peat bogs are not abundant, but lakes are widespread, although many are saline and not a few experience periodic desiccation and strong oscillations of the water table. These are, doubtless, factors linked with oxidation processes. Equipment to drill permanent lakes is expensive, limiting access to depositional centres of continuous sedimentation. Only during the last decade have funding and co-operation allowed both Spain and Portugal to carry out deep lake drilling within national and international research programmes. For example, during three months in 2004, the Pyrenean Institute of Ecology (IPE)-CSIC carried out, for the first time in Spain, a large drilling expedition (LIMNOCLIBER) throughout the Iberian Peninsula in collaboration with the Limnological Research Centre (LRC) of the University of Minneapolis (USA), with a final result of more than $200 \mathrm{~m}$ of lacustrine sediments from eight Spanish lakes. Yet a number of possibly useful lakes and marshlands have not even been drilled. An example is the Laguna de La Janda in Cádiz, one of the more extensive tectonic depressions of Iberia (Dueñas and Recio 2000), where, to our knowledge, no palynologist has yet ventured. Further cases come from the mountains, like Sierra Nevada, the Cantabrian Mountains and the Pyrenees, where high-elevation lakes appear suitable for palaeoenvironmental studies. Problems of accessibility persist for some basins, but the potential is still there. The high number of endorheic lakes in La Mancha provinces of Albacete, Ciudad Real, and Cuenca, even Jaén in Andalucía, should not be neglected in spite of the discouraging results of Pétrola, Ontalafia, and El Acequión in Albacete. Many are low-salinity and nearly permanent, and peat deposits are sometimes preserved at their margins (Cirujano 1990; Casado and Montes 1995). The region contains archaeological and coprolite sites in abundance but the former often fail to contain pollen, and the latter have been insufficiently tested.

It is also worth wondering whether failures with pollen analysis have been equally common in other territories, but have simply not been reported. Collecting the data presented here has been time-consuming, and no doubt some would regard such an exercise as producing little career reward for the effort. Apart from the severe difficulties in getting active collaboration, there have been cases among the contributors where the laboratories have been demolished, or where the researcher has been moved and the original processing sheets have been impossible to rescue. So, with fragmentary information, we are aware that this work is incomplete in many aspects and needs further detail before we can achieve more far-reaching conclusions. This is a first step only. The next step is to stimulate future controlled investigation of negative results, a more multidisciplinary approach, more frequent collaborative research between palaeoecologists, and, necessarily, a more realistic assessment among Quaternary specialists of what information palynology can give and what it is unable to deliver. 

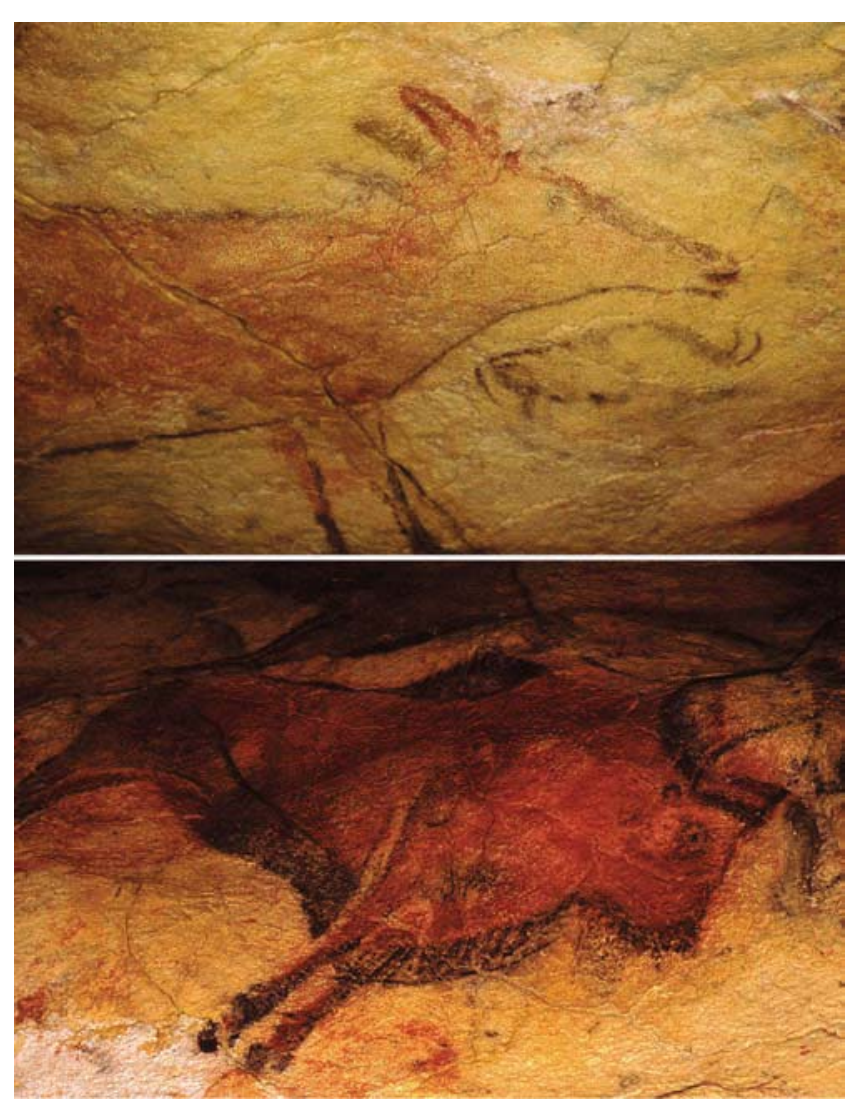

Figure 4. Altamira. Palaeolithic wall paintings of Altamira, Cantabria, northern Spain. Photographs: J.S. Carrión.

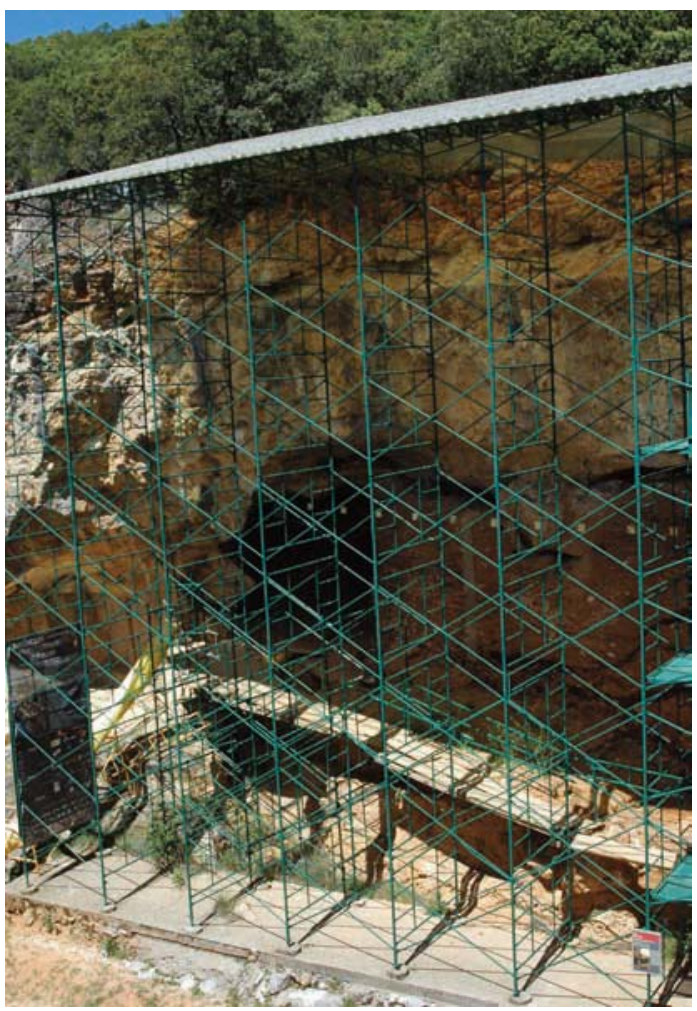

Figure 6. Atapuerca Galería. Excavation in Atapuerca Galería. Photograph: M. García-Antón.

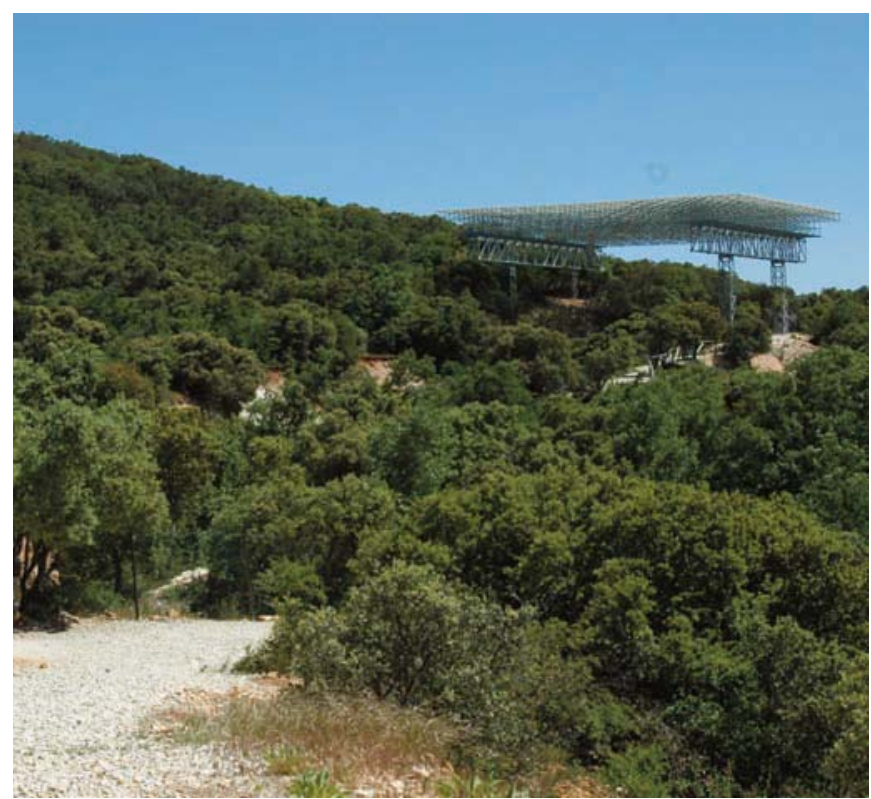

Figure 5. Atapuerca Sima de los Huesos. Exterior view of excavation in Atapuerca Sima de los Huesos. Photograph: M. García-Antón.

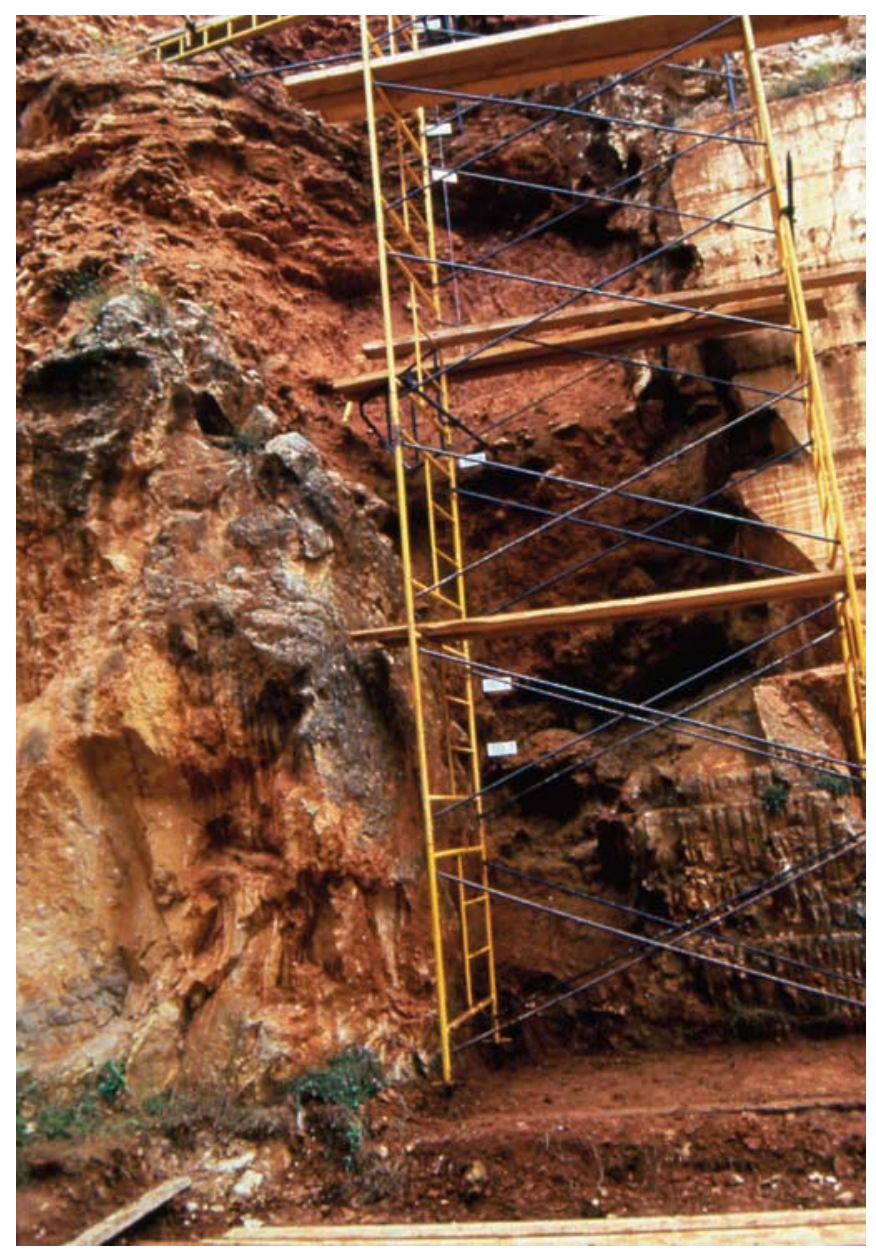

Figure 7. Atapuerca Gran Dolina. General view of Sections TD1, TD-2, TD-3 in Atapuerca Gran Dolina. Photograph: M. García-Antón. 


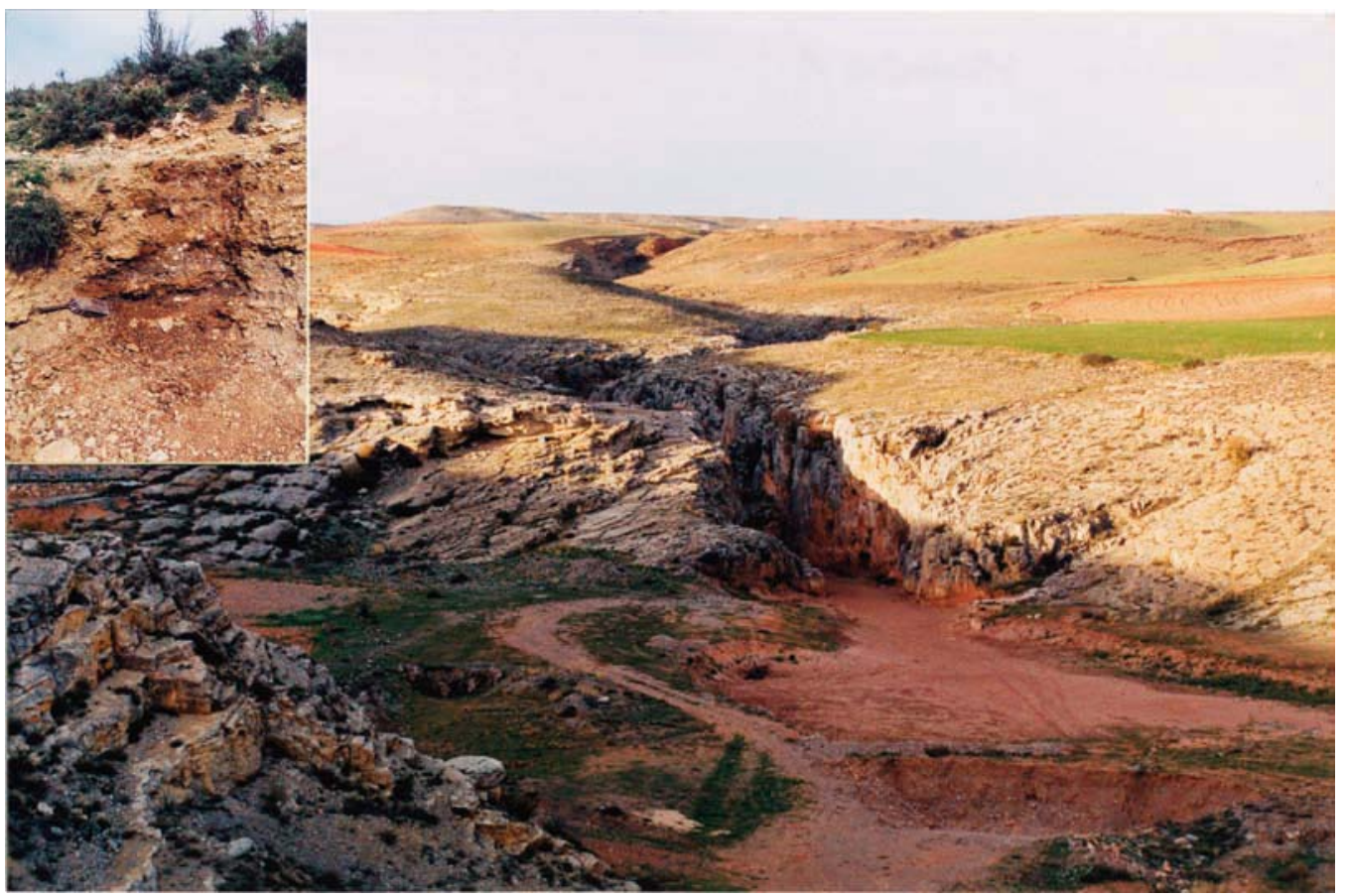

Figure 8. Barranco Hondo. A slope deposit palynologically unproductive in Barranco Hondo, Teruel. Photograph: P. González-Sampériz.

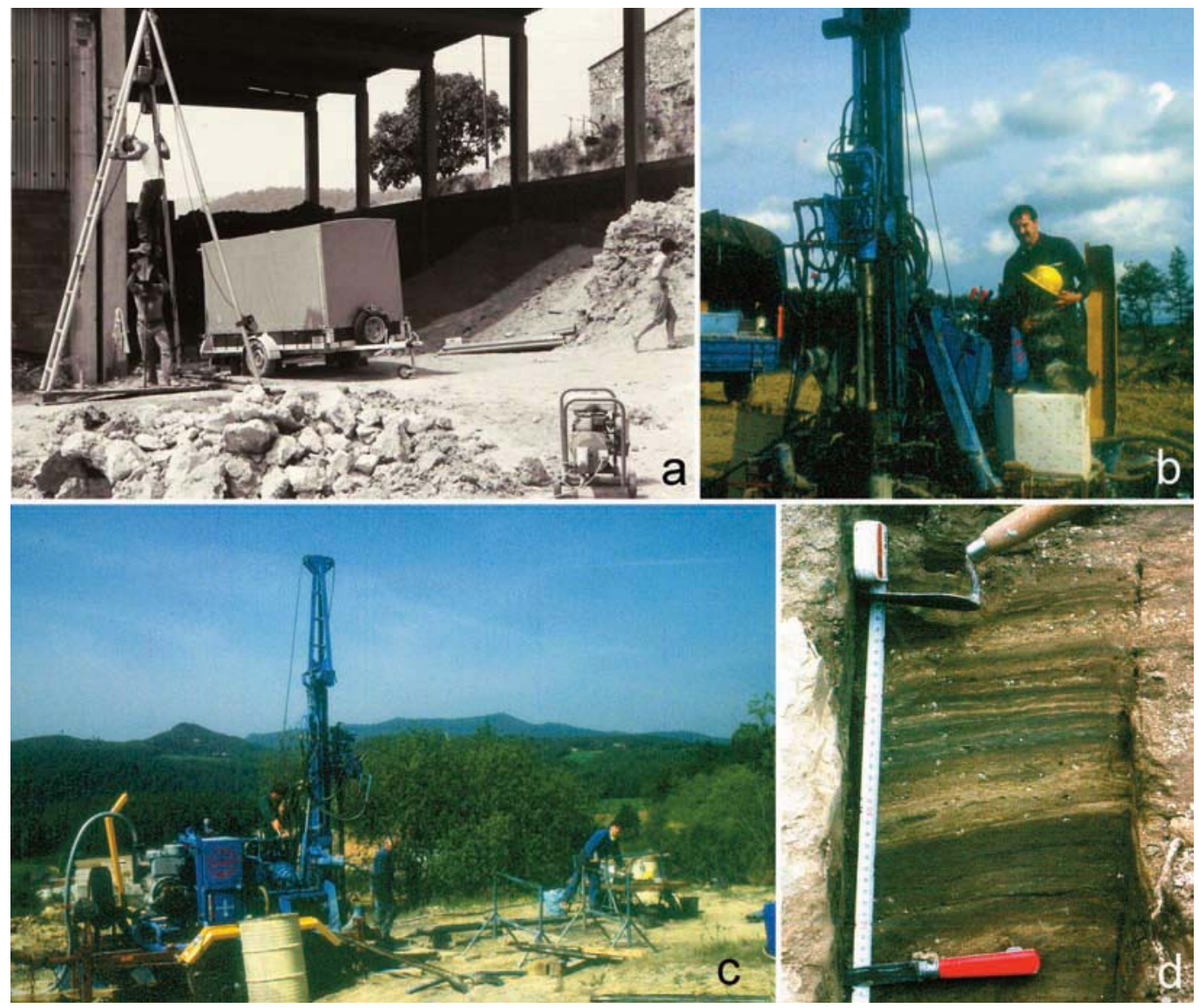

Figure 9. Bòbila Ordis. a) Coring in the brickyard of Bòbila Ordis (core II) in 1983. b-c) Coring of Bóbila Ordis (core IV) in 1988. d) Sediment in outcrop of Bòbila Ordis (Lake 2) showing the injection of older sediment along the fault plane. Photographs: S. Leroy. 


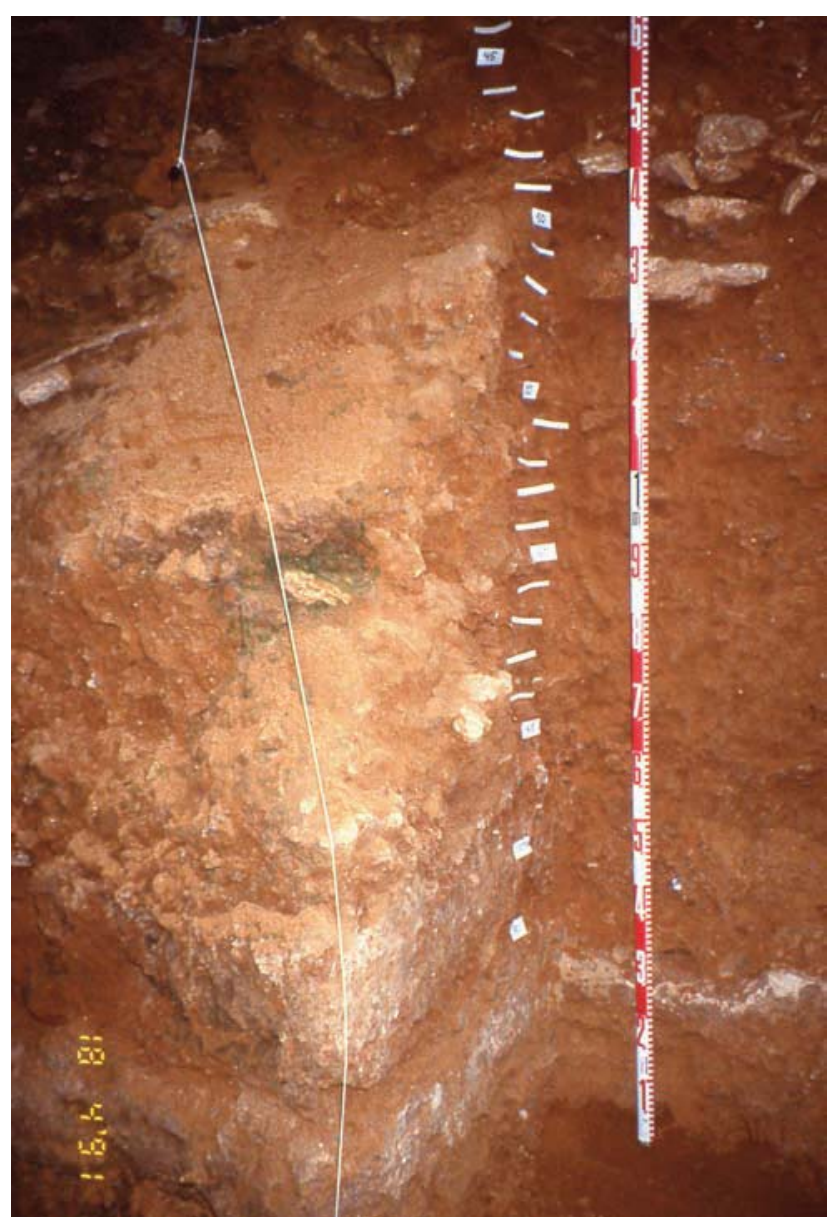

Figure 10. Bolomor. Main stratigraphical section of Cova Bolomor, a renowned Pleistocene cave site of eastern Spain. Photograph: M. Dupré.

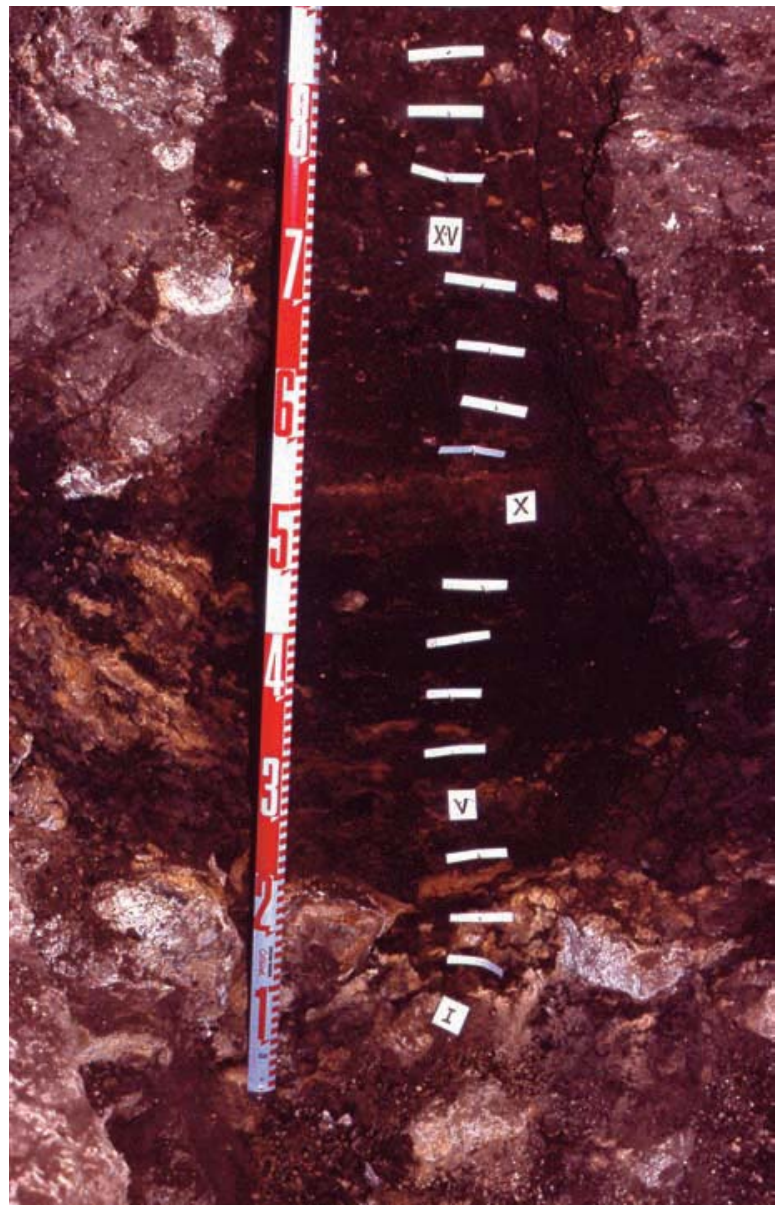

Figure 11. Carihuela. Carihuela Cave Chamber III Section 2 (Corte de Ico) showing the location of pollen samples in 1988. Most dark layers, despite their high organic content, were sterile. Photograph: J.S. Carrión.

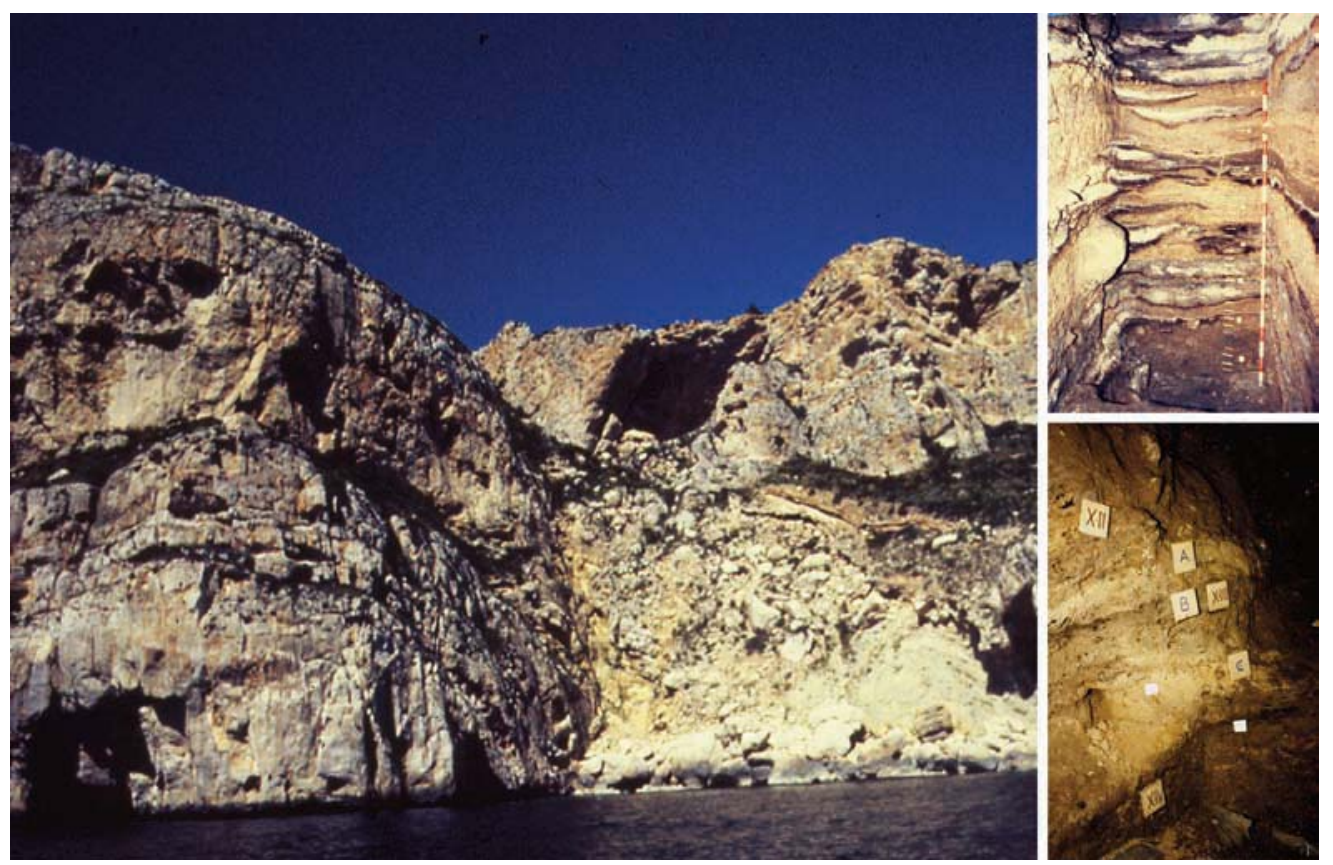

Figure 12. Cendres. Panoramic view and sections studied for pollen in Les Cendres cave (Alicante, Mediterranean littoral). Photographs: M. Dupré. 


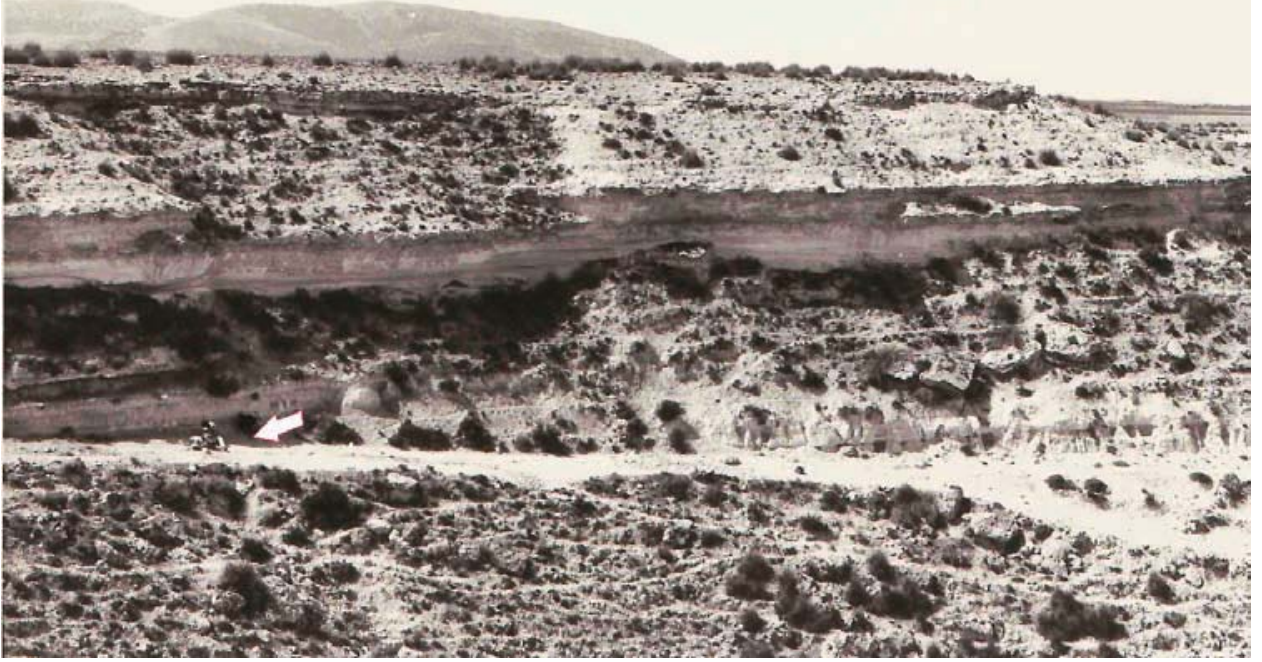

Figure 13. Conejos. The Barranco de los Conejos gully in the Orce region, Granada. Scale given by person in the lower left. Photograph: S. Leroy.
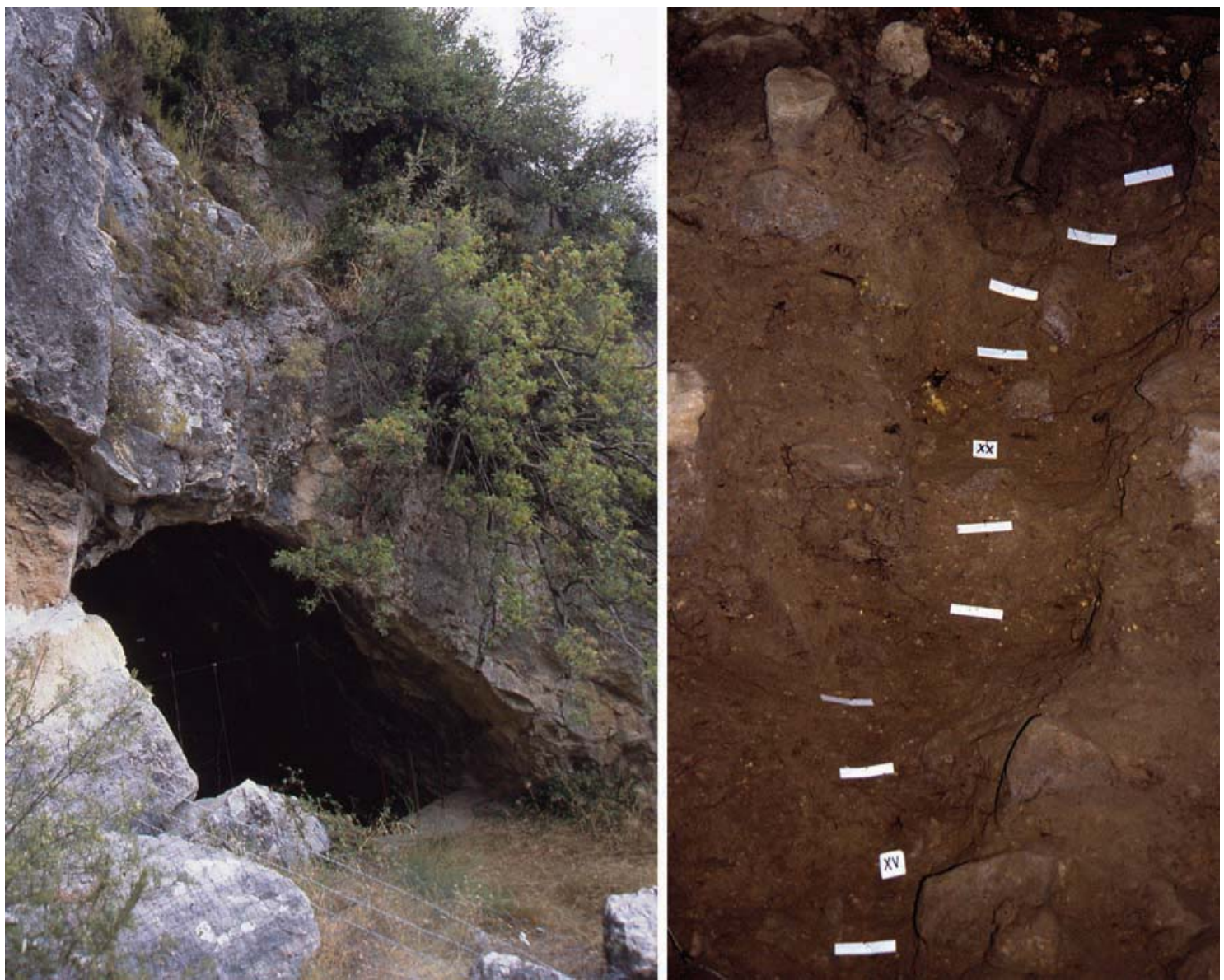

Figure 14. Cova Beneito. Entrance area and upper Palaeolithic section of Beneito cave, Alicante. Photographs: J.S. Carrión. 


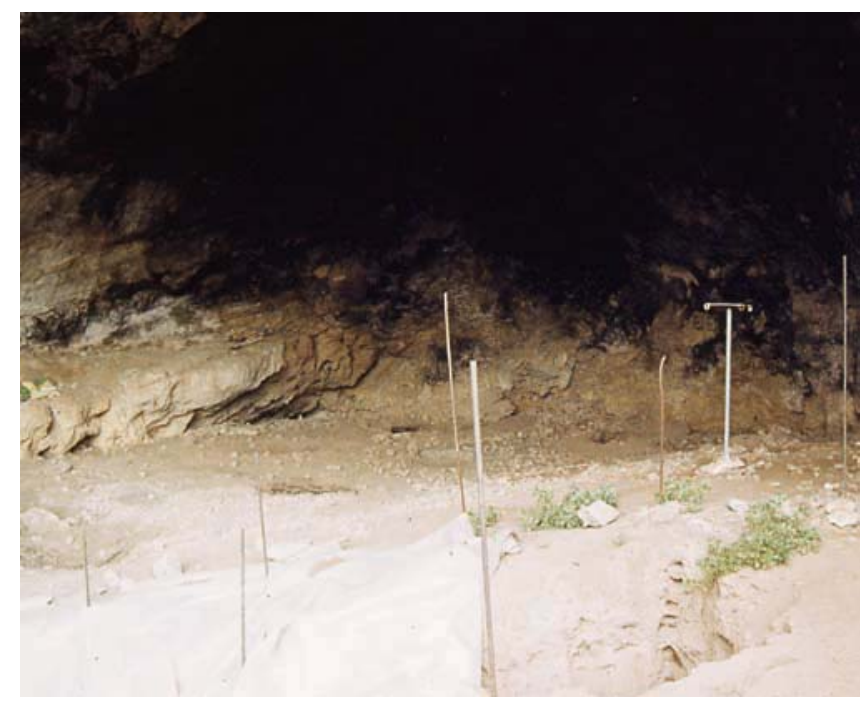

Figure 15. Cova Negra. The Mousterian cave of Cova Negra, Játiva. Photograph: M. Dupré.
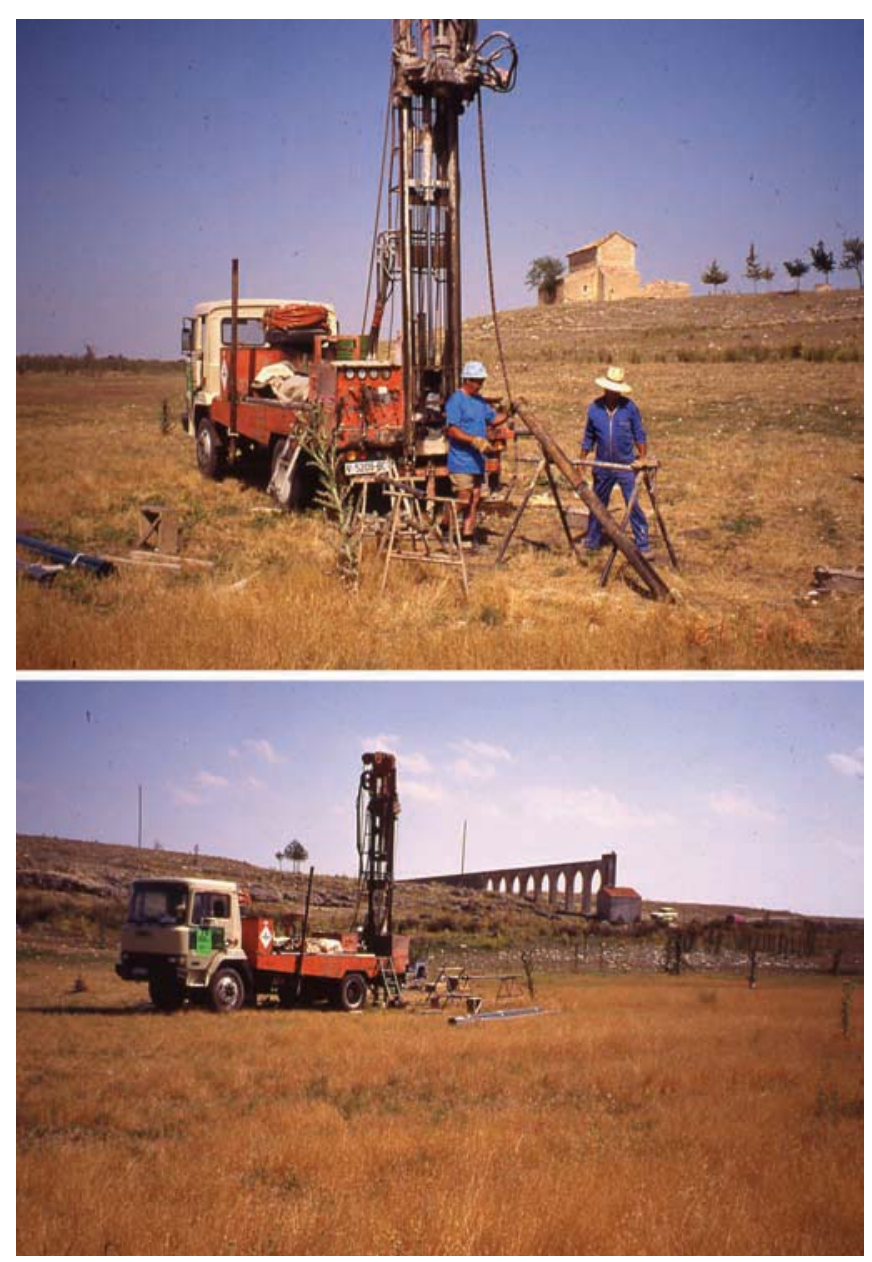

Figure 17. El Acequión. Drilling with hydraulic piston corer the Holocene sediments of Laguna del Acequión salt lake in Albacete. All the pollen samples were sterile. Photographs: M. Dupré.

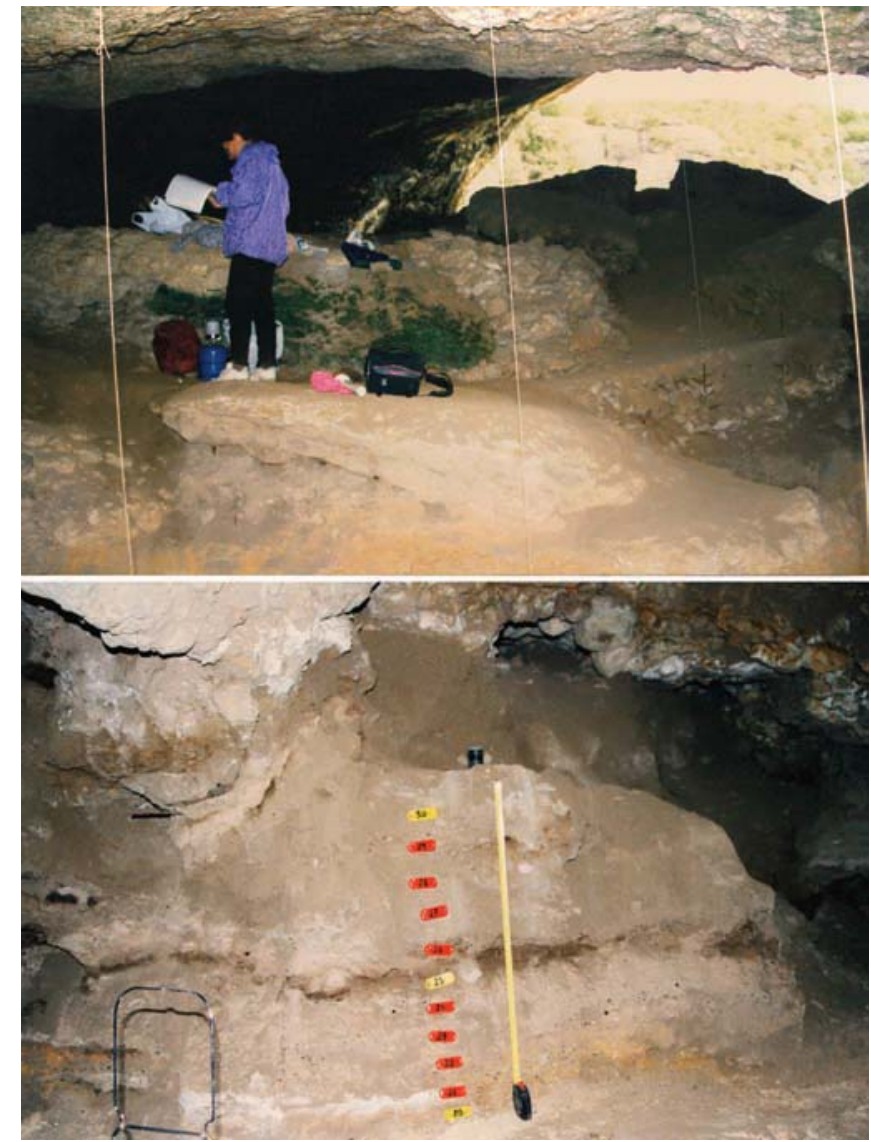

Figure 16. Chaves. General view and stratigraphical section from Chaves cave, Huesca. Photographs: P. González-Sampériz. 


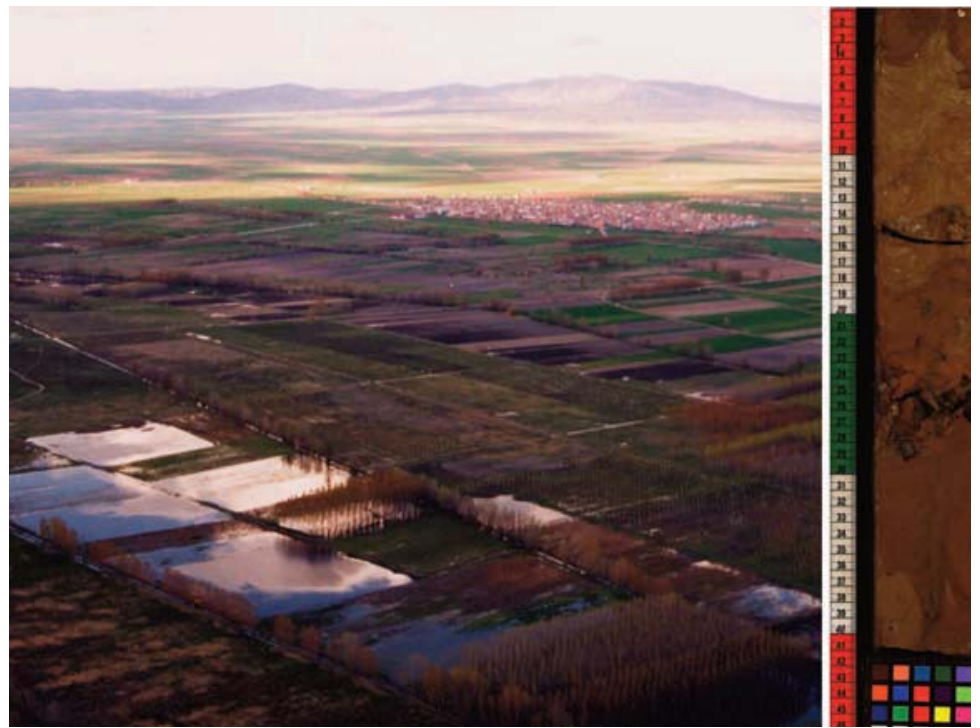

Figure 18. El Cañizar, Villarquemado. Deep coring in the Laguna del Cañizar, Villarquemado, Teruel. Photograph: P. González-Sampériz.

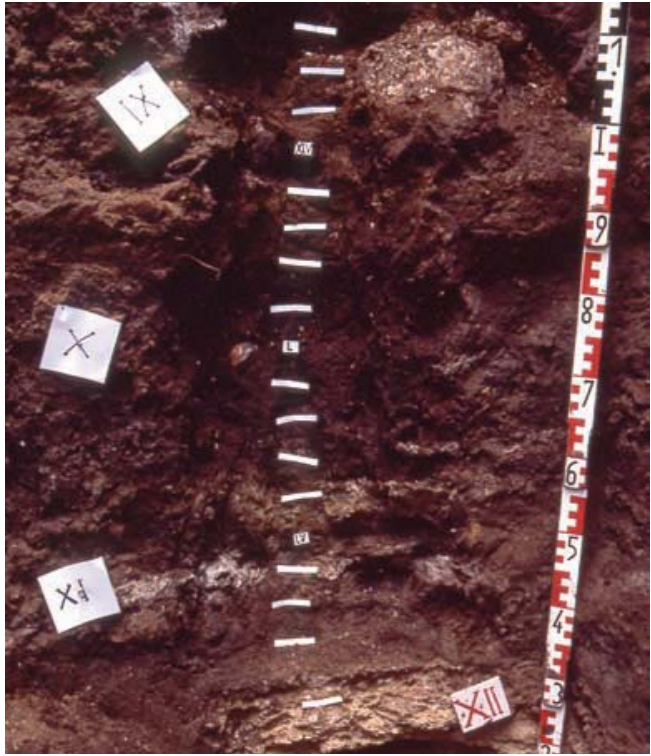

Figure 19. El Salt. Stratigraphical section of the Mousterian cave site of EI Salt, Alicante. Both the stalagmitic crusts (below) and the darker, more organic levels were palynologically sterile. Photograph: M. Dupré.

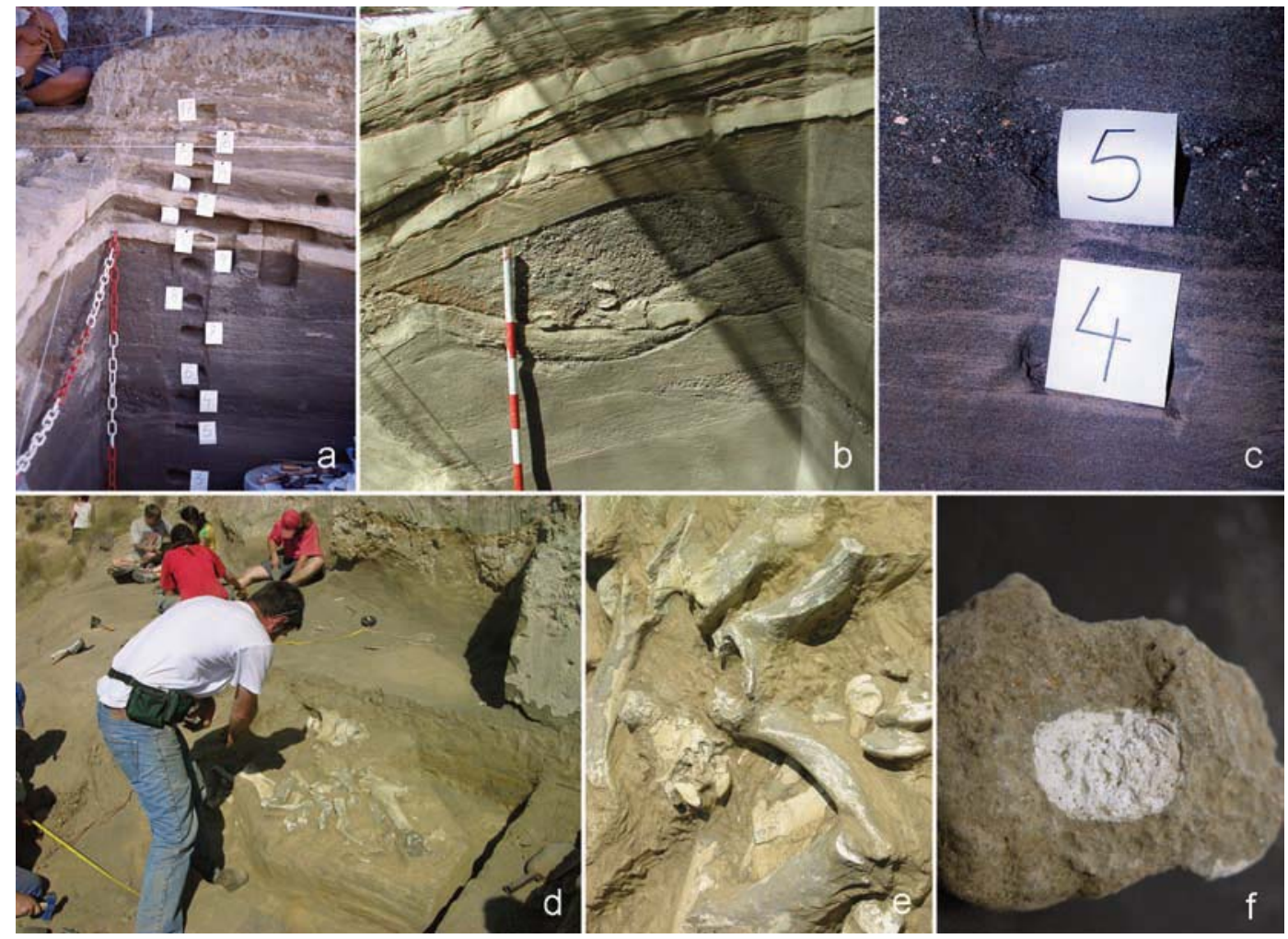

Figure 20. Fonelas. The Upper Pliocene palaeontological site of Fonelas, Guadix Basin, very rich in mammal bones (d-e). Sediment samples (a-c), mostly of coarse fraction, and coprolites of the hyaenid Chasmaporthetes were palynologically sterile. Photographs: J. S. Carrión \& S. Fernández. 


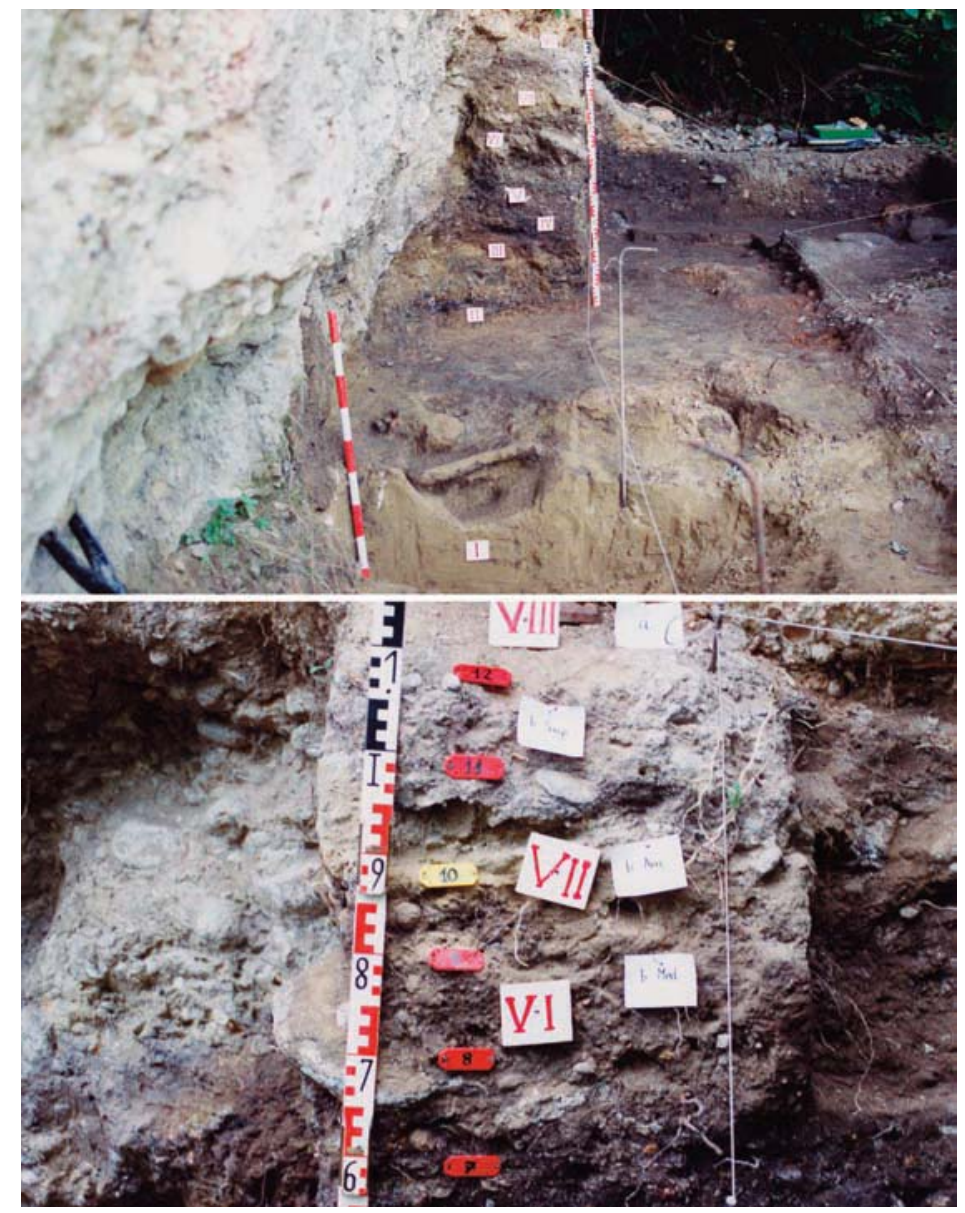

Figure 21. Forcas. Stratigraphical section considered for pollen in the Forcas rockshelter, Huesca. Photographs: P. González-Sampériz.

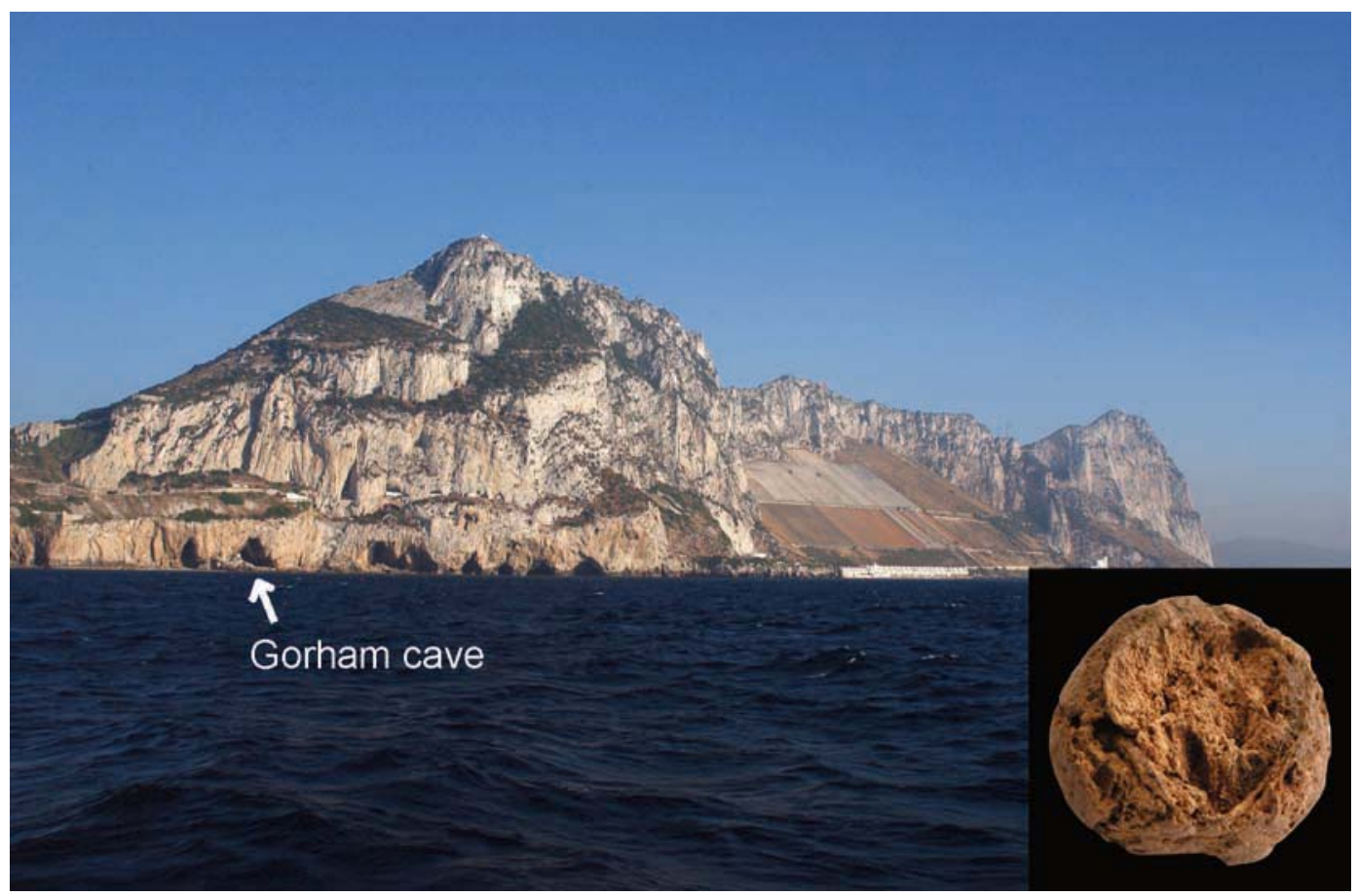

Figure 22. Gorham's Cave. The Palaeolithic levels of Gorham's Cave, Gibraltar Peninsula, have provided a number of coprolites (below right), presumably from hyaenids and canids. Some of these have been polliniferous while, inexplicably, others were totally barren of pollen. Photographs: C. Finlayson \& J.S. Carrión. 


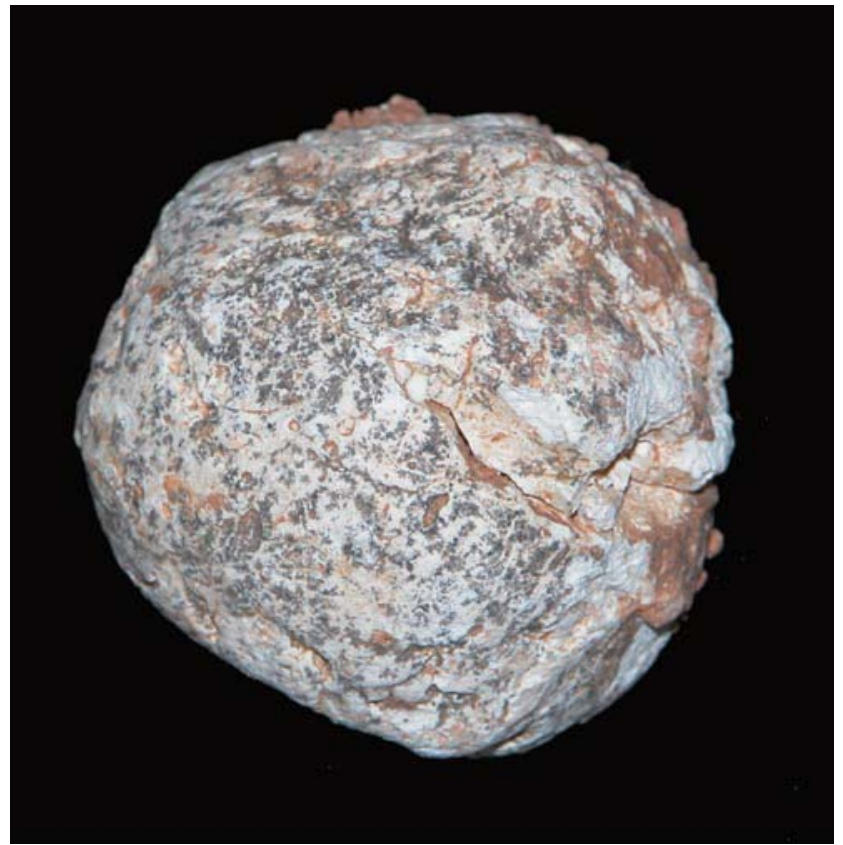

Figure 23. Grajo. Crocuta coprolite from Cueva del Grajo, Córdoba. Photograph: S. Fernández.
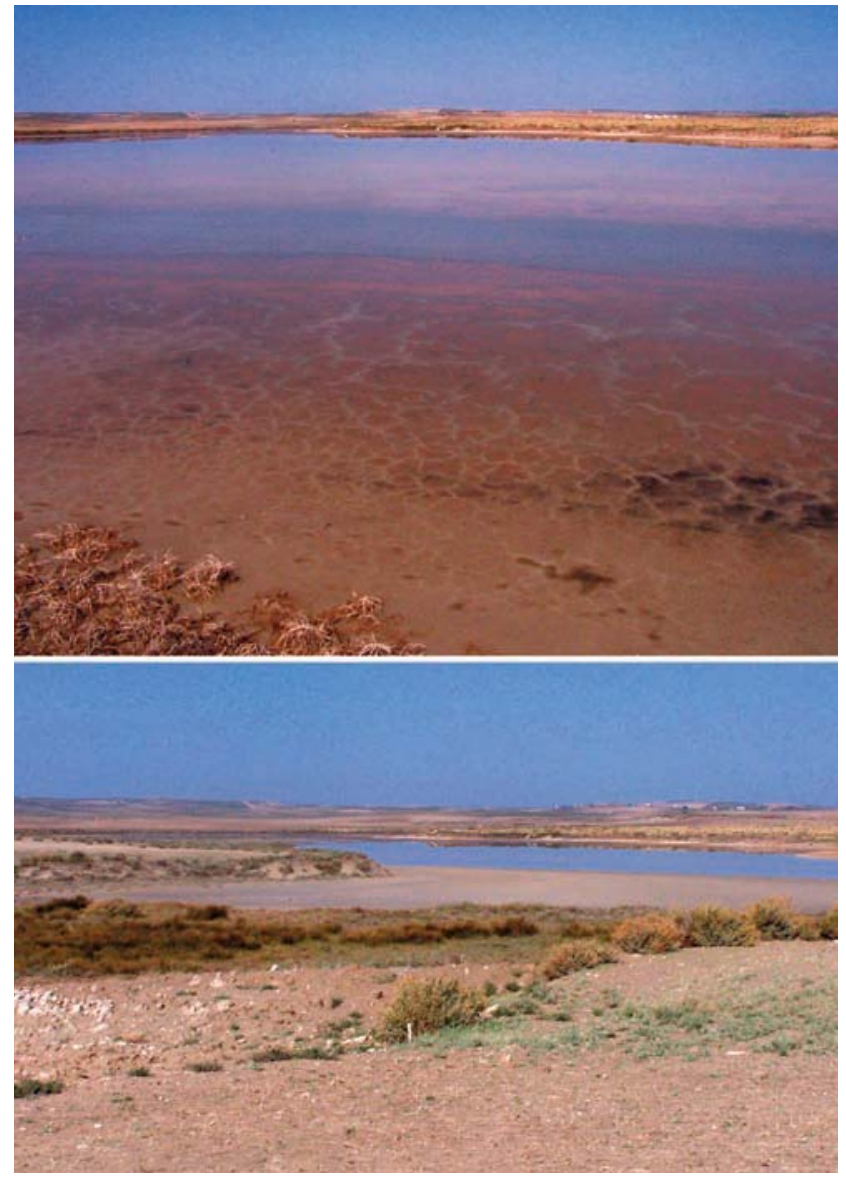

Figure 25. La Playa. Playa lake of La Playa, north-eastern Spain. Photograph: P. González-Sampériz.

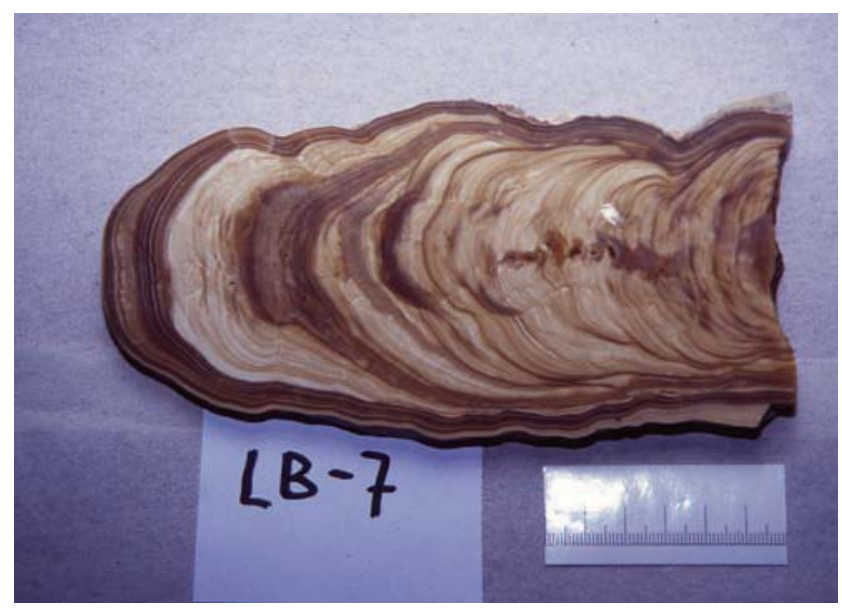

Figure 24. La Blanca. Longitudinal section of one of the several palynologically sterile calcium carbonate cave speleothems from La Blanca. Photograph: J. Carrión.

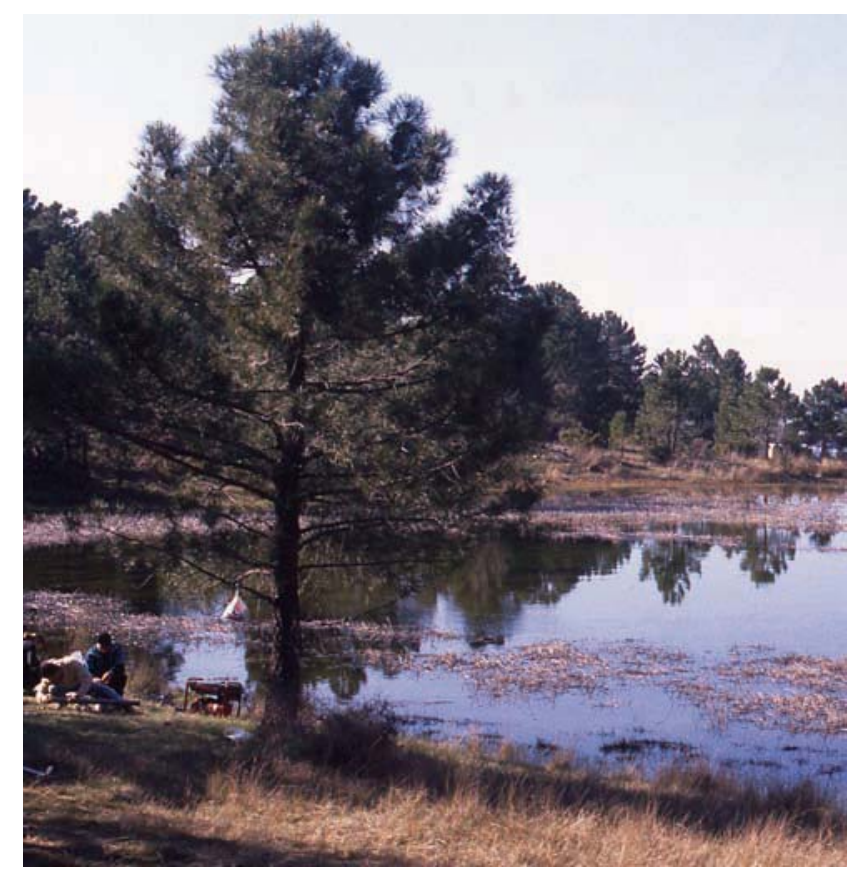

Figure 26. Laguna de Orcera, Segura Mountains of southern Spain. A sediment core from this lake was palynologically sterile. Photograph: J. Carrión. 

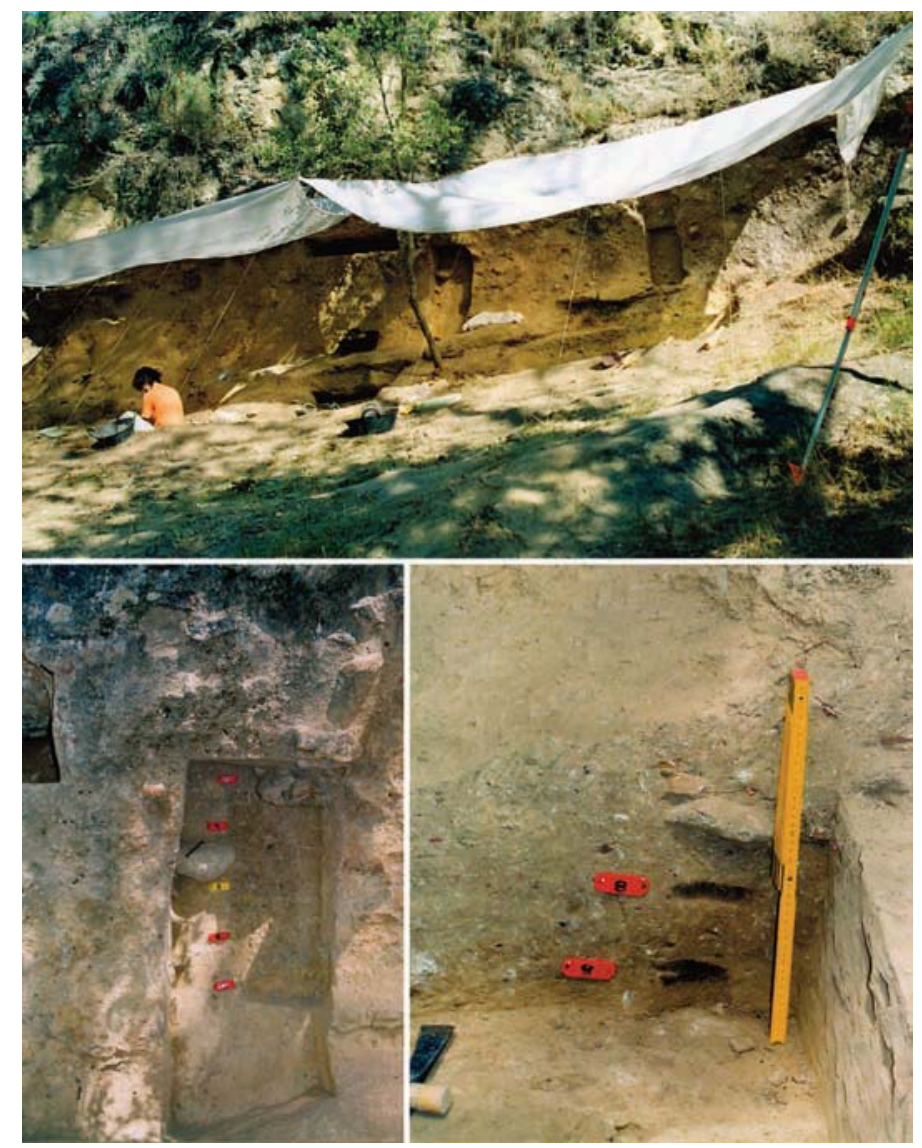

Figure 27. Legunova. The partially sterile Legunova rockshelter, an Azilian to Neolithic site of northern Spain. Photograph: P. González-Sampériz.

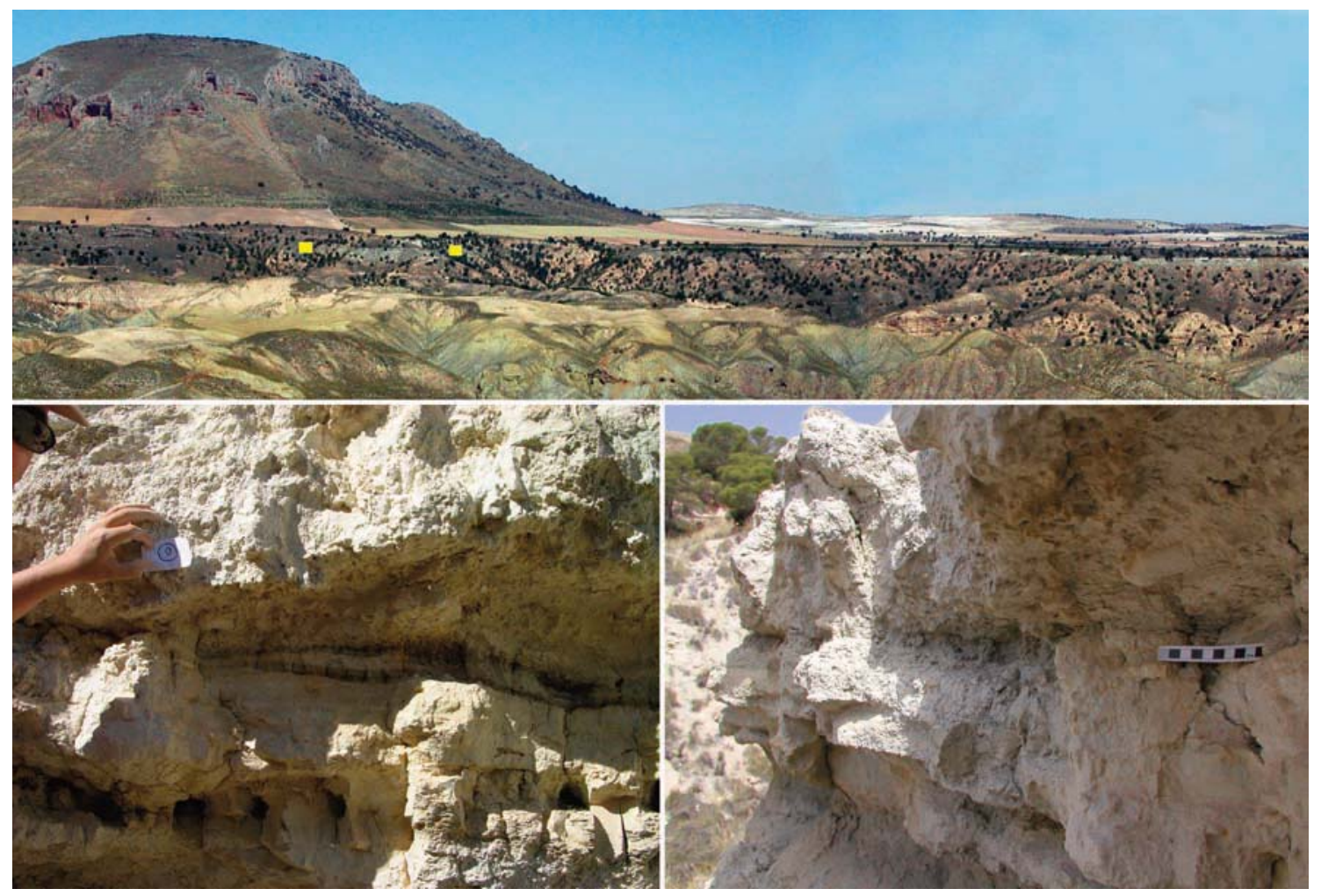

Figure 28. Mencal. The recently discovered large mammal fossil site of Mencal, Guadix Basin, semi-arid south-eastern Spain. Fossiliferous micrites and lutites (below) originated in Upper Pliocene lacustrine environments and were palynologically sterile. Photographs: S. Fernández and A. Arribas. 


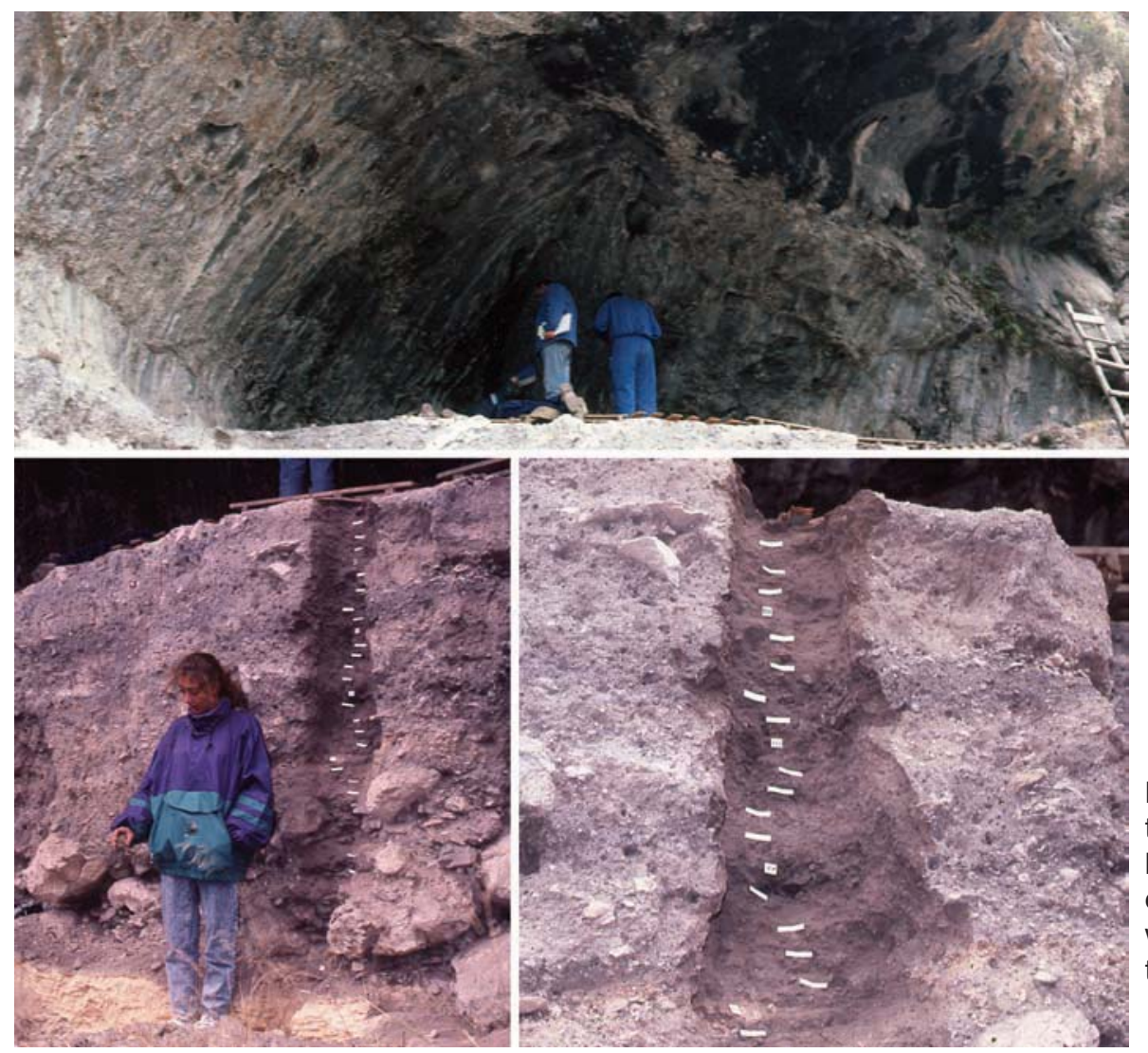

Figure 29. Molino del Vadico. The Neolithic rockshelter of Molino del Vadico, Albacete. Pollen grains were absent from all deposits. The section studied (below) showed abundant insect and root bioturbations. Photographs: J.S. Carrión.

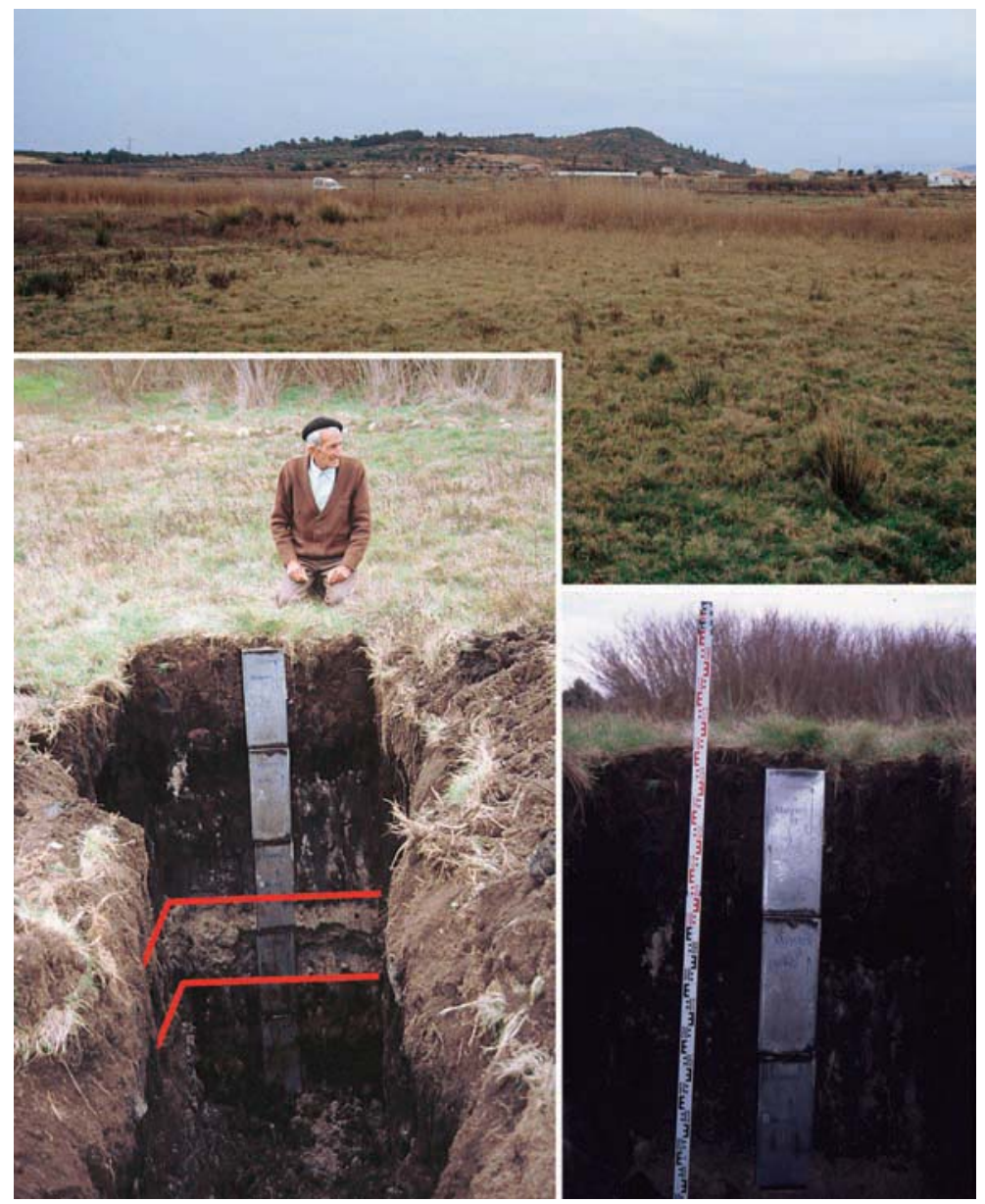

Figure 30. Navarrés. The Navarrés peatbog produced a long pollen sequence from OIS3 to late Holocene. However, the OIS2, pleniglacial levels, dominated by aeolian sands (red lines), were palynologically sterile. Photographs: J.S. Carrión. 


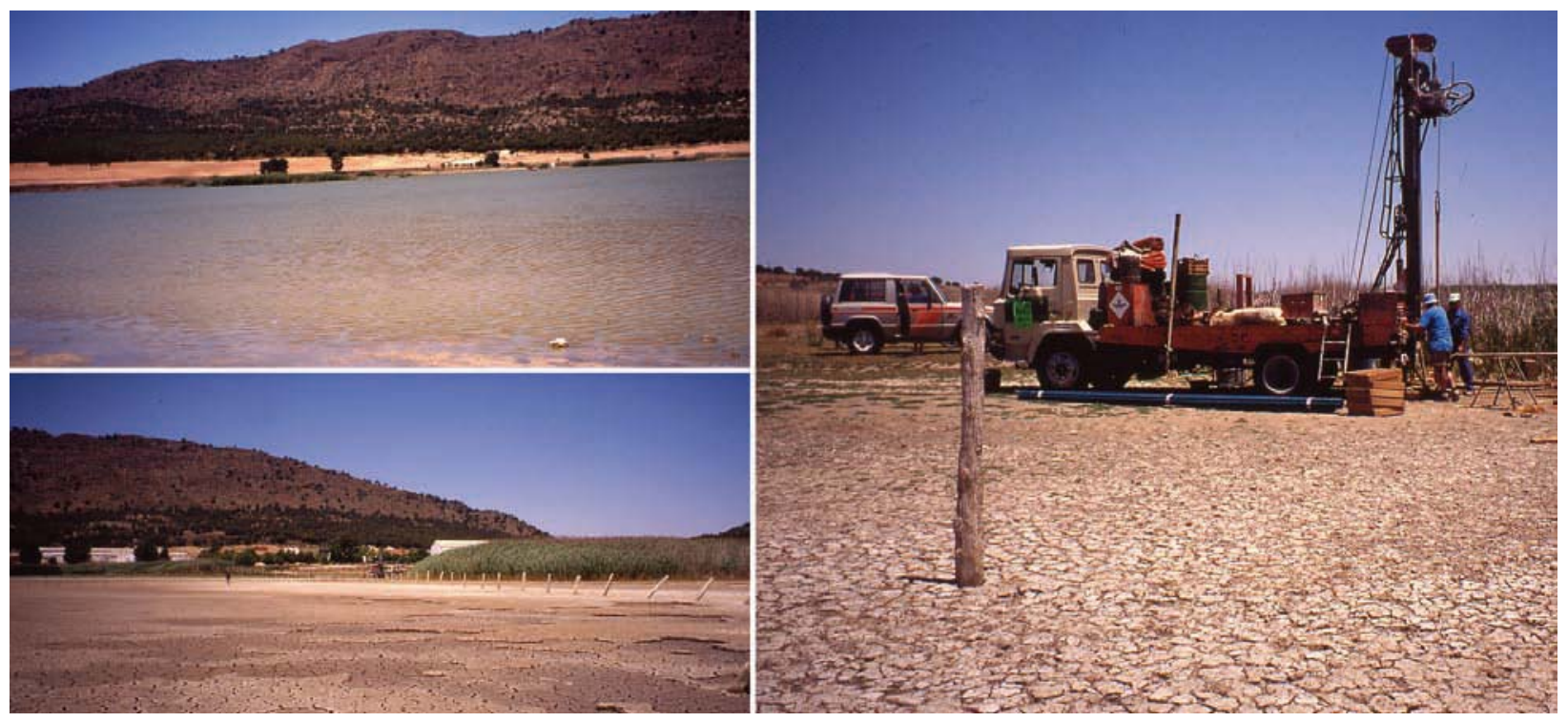

Figure 31. Ontalafia. Inundated and dry Ontalafia salt-lake in La Mancha Plain, central Spain. All sediment cores were palynologically sterile. Photographs: M. Dupré.

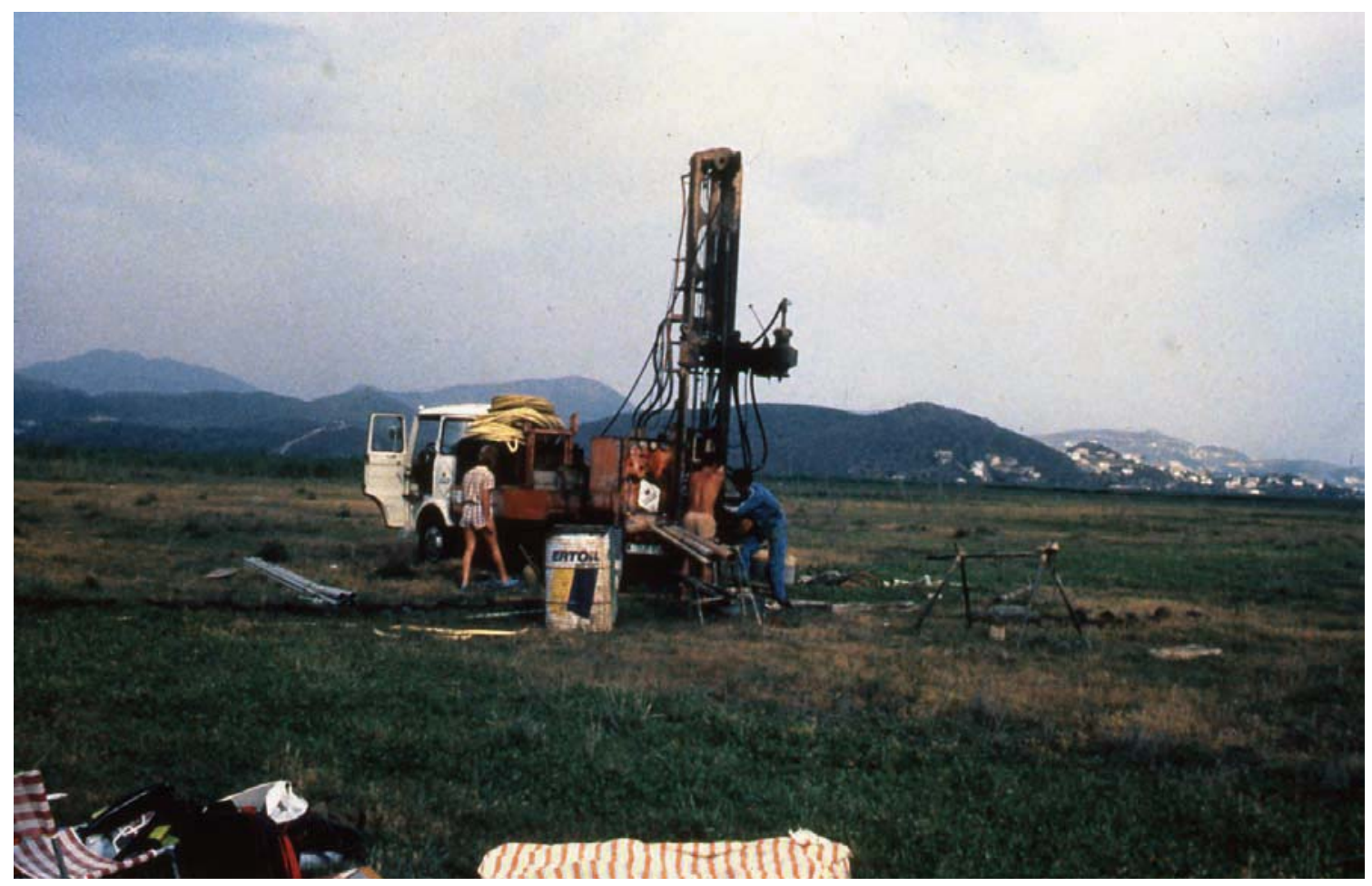

Figure 32. Pego. Pollen analyses of two coreholes in the Pego-Oliva littoral marsh were scarcely rewarding. Pollen was poorly preserved, and episodically absent from quite an organic-rich, yet salty, sediment. Photograph: M. Dupré. 


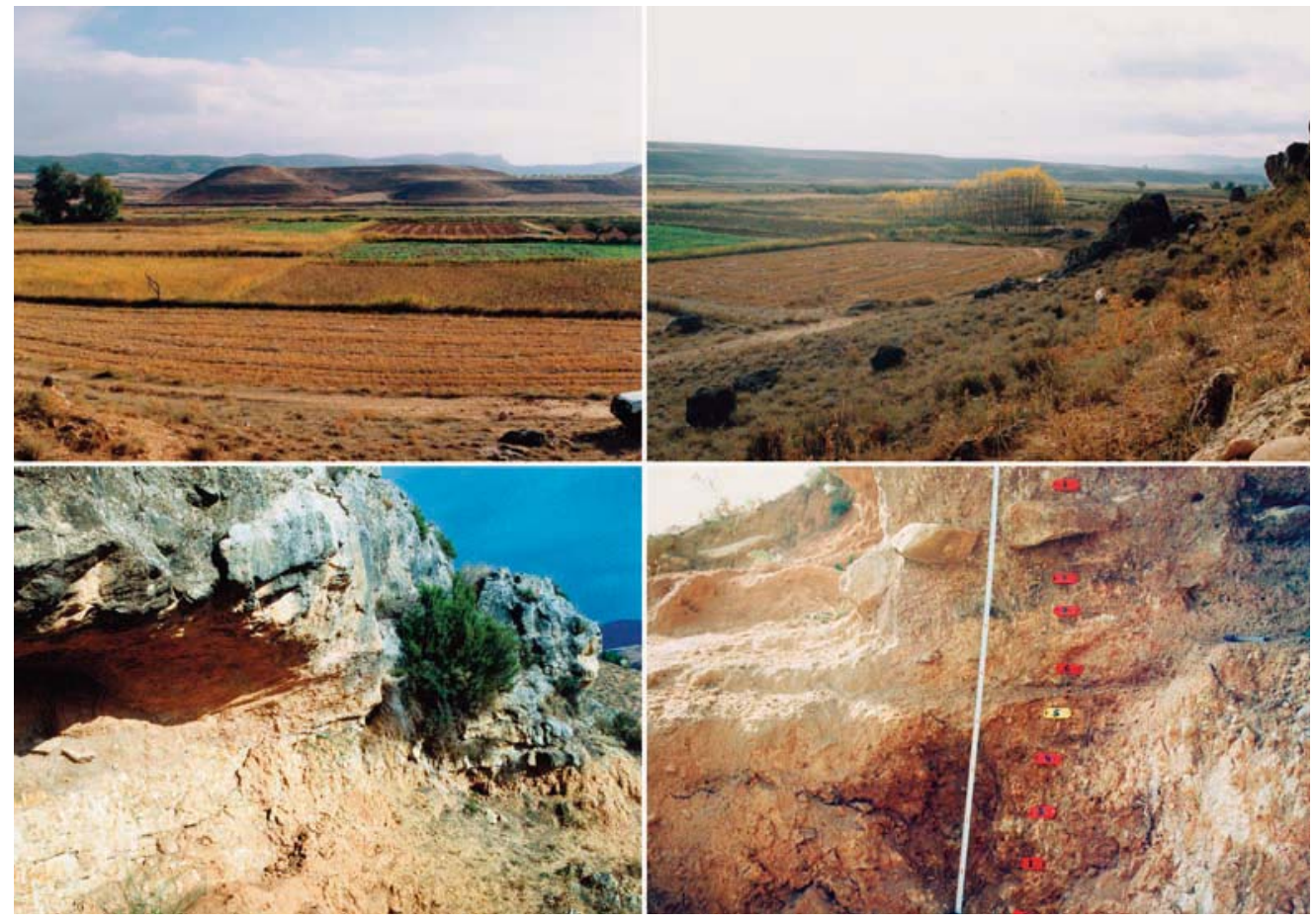

Figure 33. Peña del Diablo rockshelter in Zaragoza province. Photographs: P. González-Sampériz.
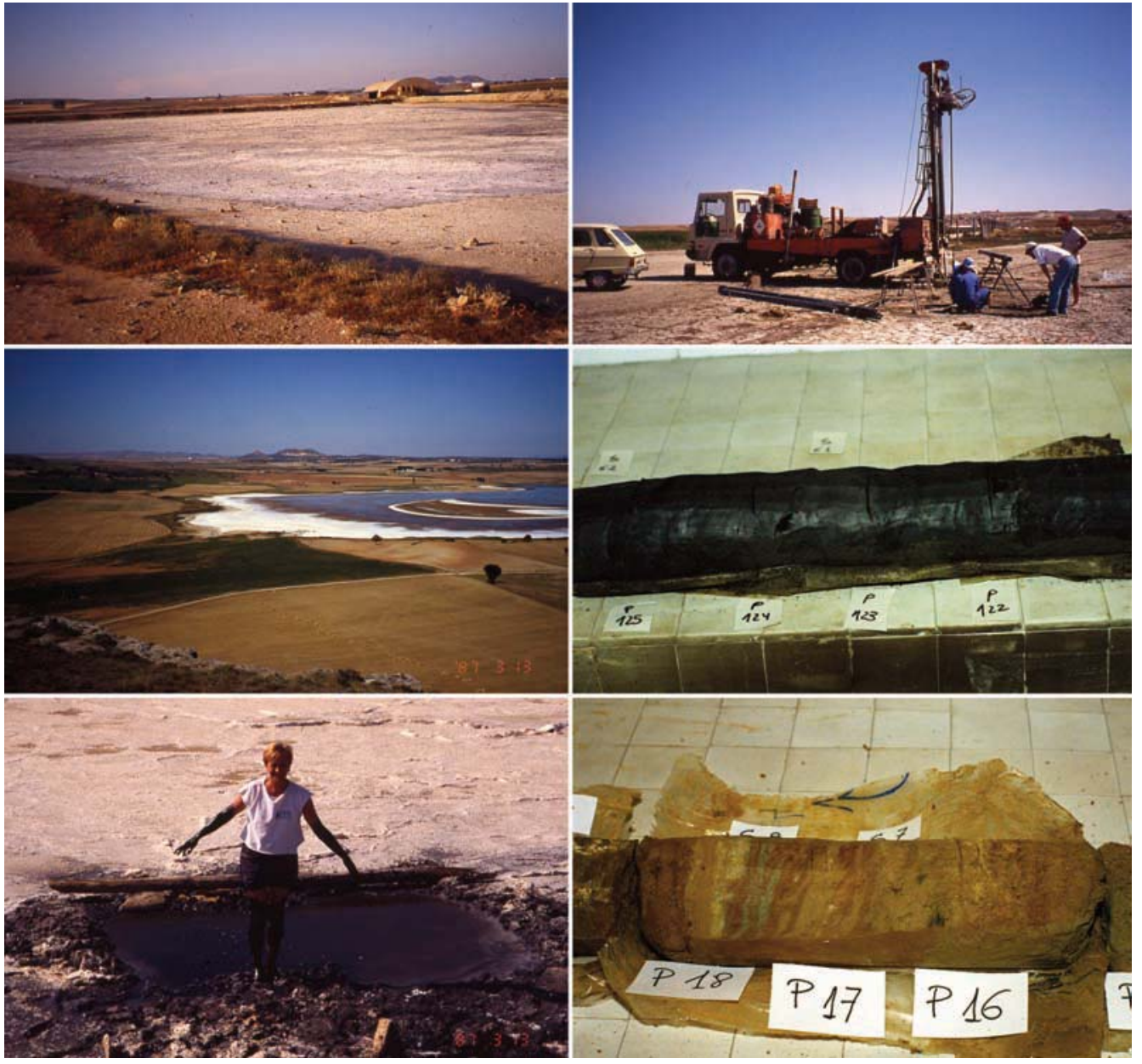

Figure 34. Pétrola. The sediments from the saline lake Pétrola were rich in carbonates and, especially, signs of oxidation were observed throughout the core, and chlorides and sulfates (anhydrite and gypsum) very common. All samples for pollen were sterile. Photographs: M. Dupré. 

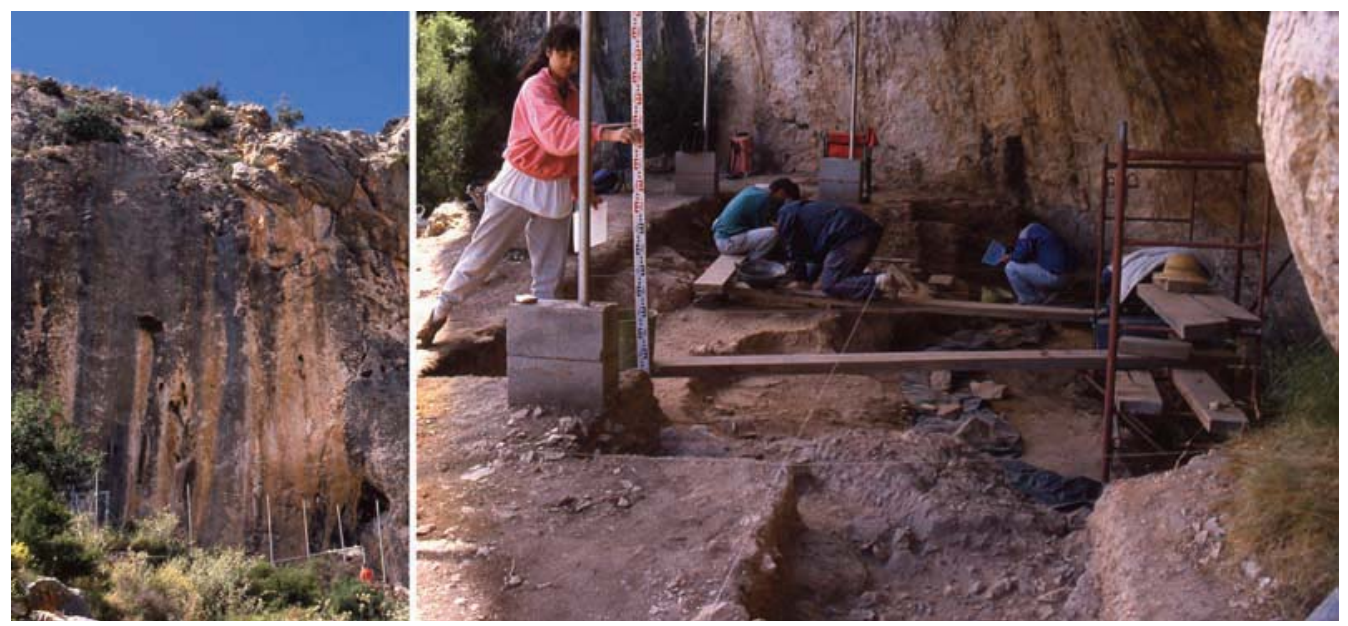

Figure 35. Ratlla del Bubo. The Upper Palaeolithic rockshelter Ratlla del Bubo, Alicante, fully sterile. Photographs: M. Dupré.
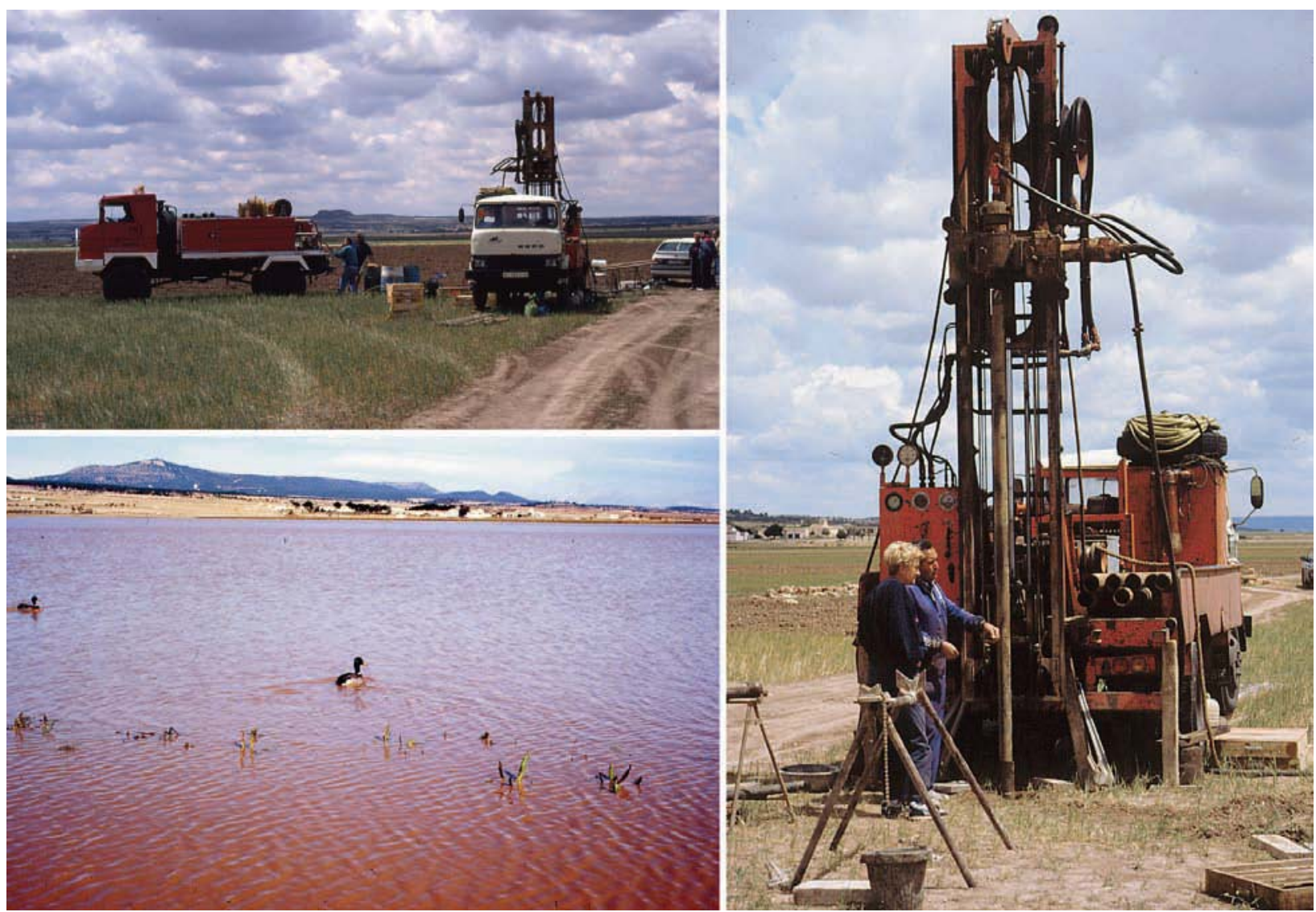

Figure 36. San Benito. San Benito seasonal lake. A sediment core gave palynologically rich levels alternating with sterile ones. Photograph: M. Dupré. 


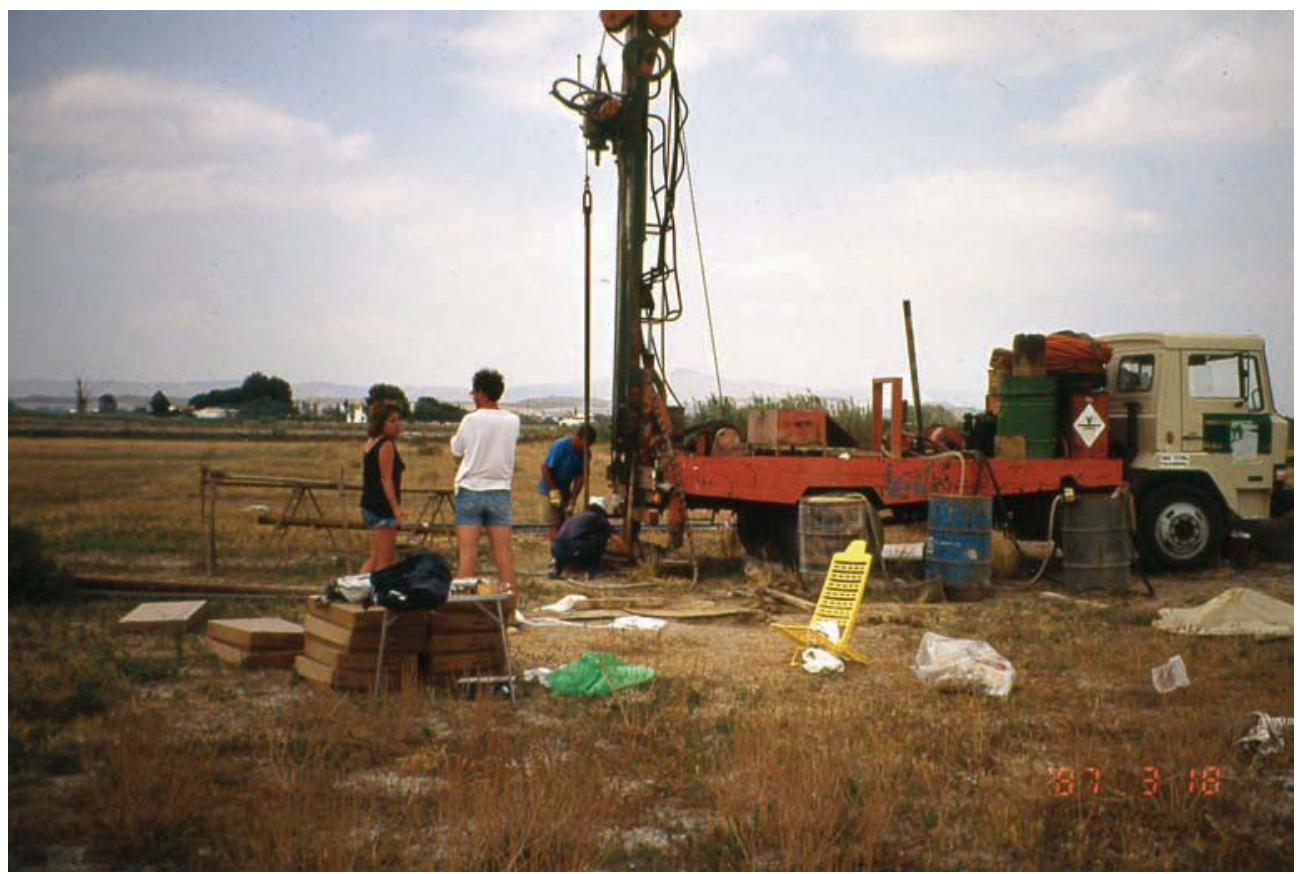

Figure 37. Torreblanca. A littoral peat bog, Torreblanca. Palynologically sterile levels occur under the influence of fluvial and marine depositional environments. Strictly paludal levels are polliniferous. Photograph: M. Dupré.
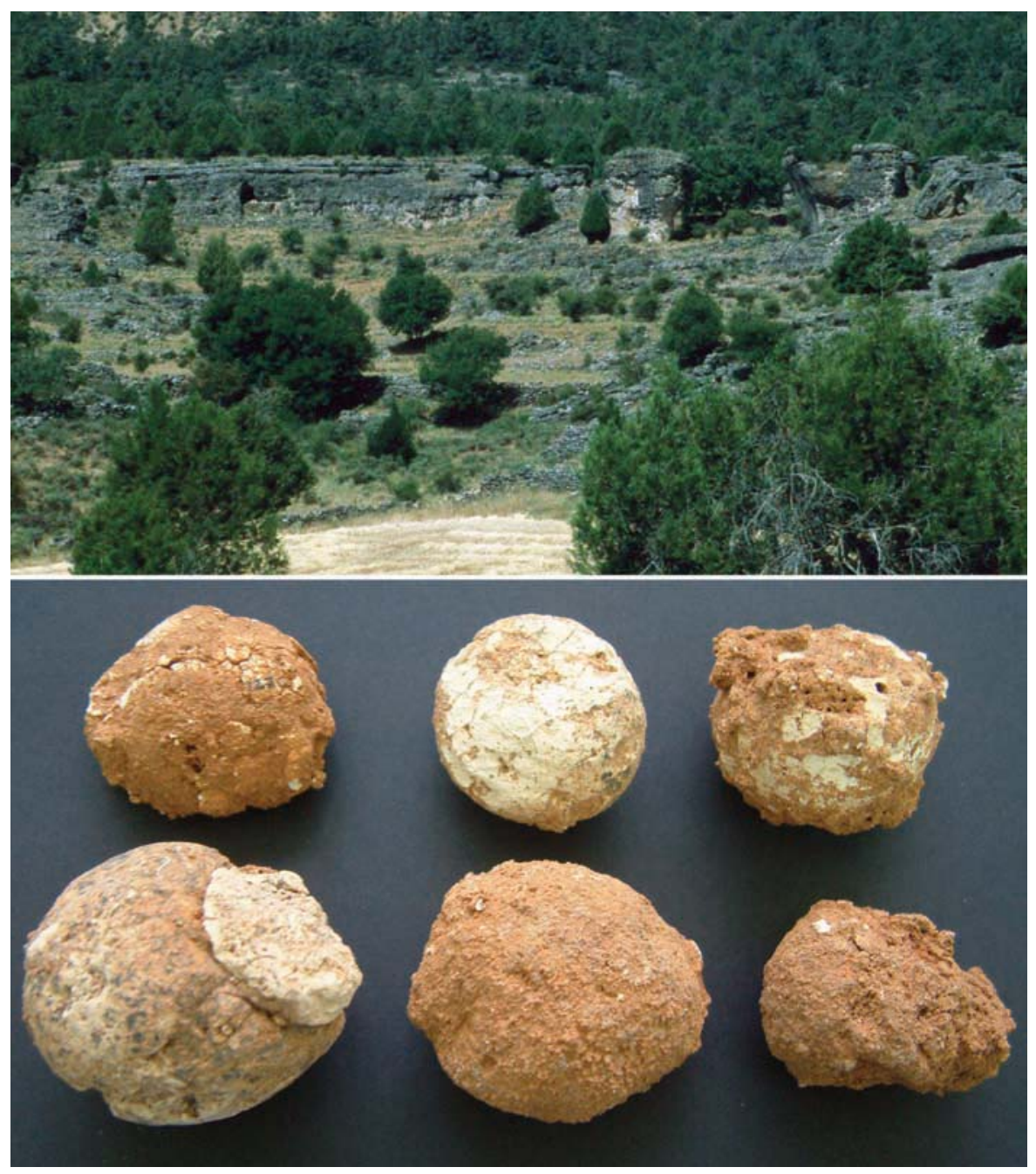

Figure 38. Torrejones. The Pleistocene infill of the Torrejones Cave (above), Central System, provided a number of hyaena (Crocuta crocuta) coprolites (below), with strong differences in their potential for palynology. Photographs: J.S. Carrión. 


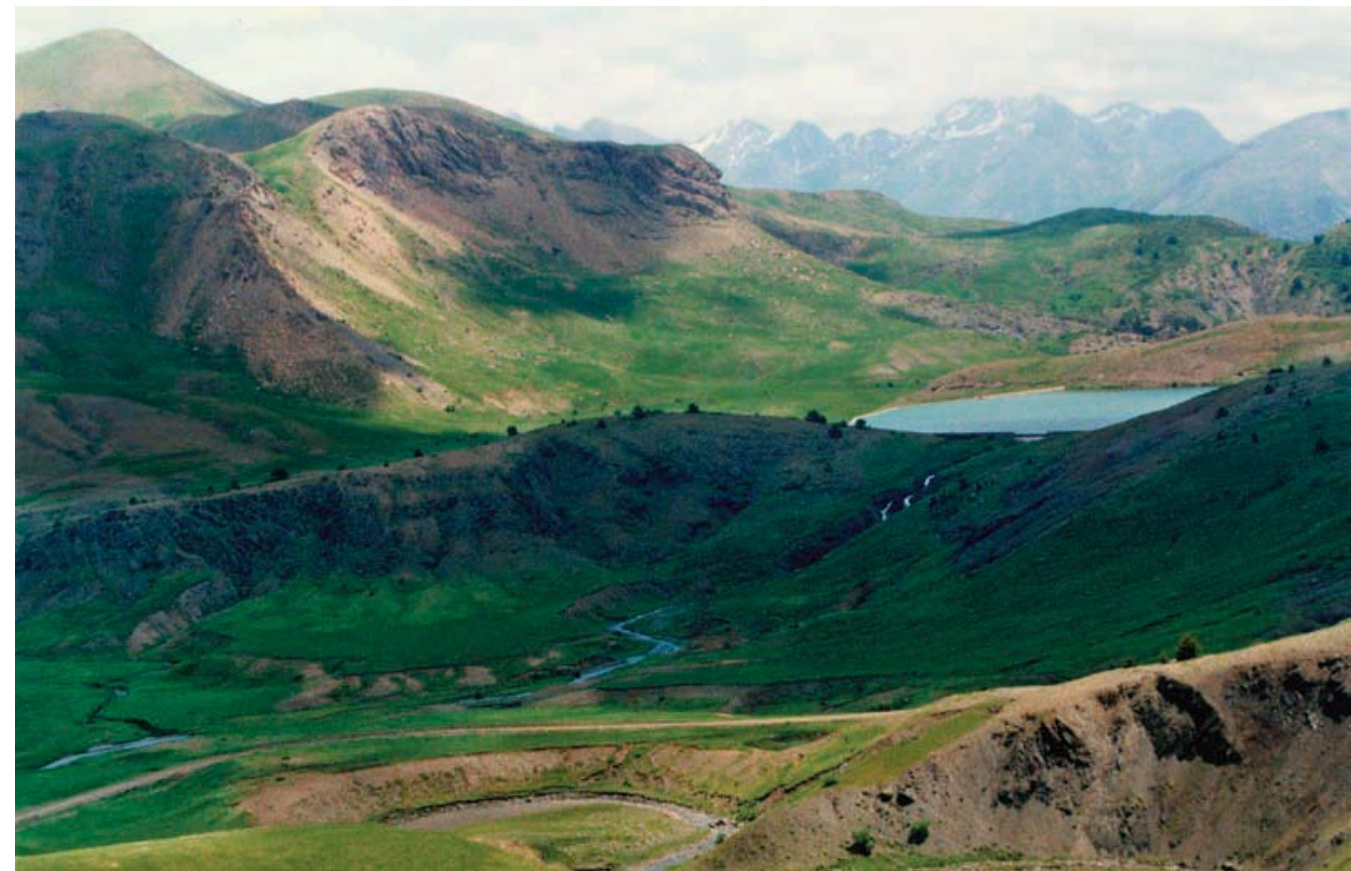

Figure 39. Tramacastilla. The moraine deposit of Tramacastilla, Huescan Pyrenees, completely sterile. Photograph: P. González-Sampériz.

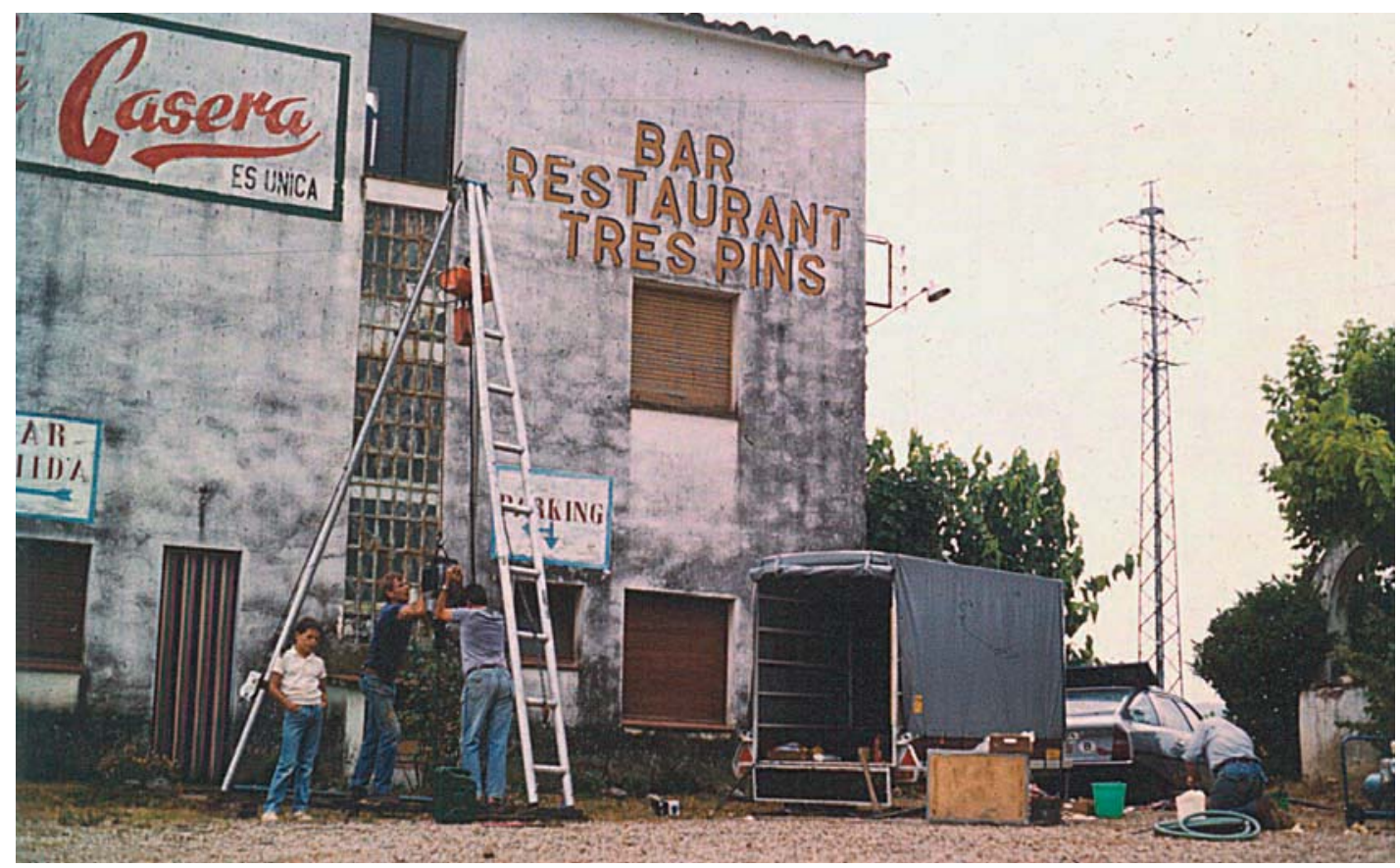

Figure 40. Tres Pins. Coring of TPI core at Tres Pins, a Upper Pliocene-Lower Pleistocene site near Banyoles, in 1983. Photograph: S. Leroy. 


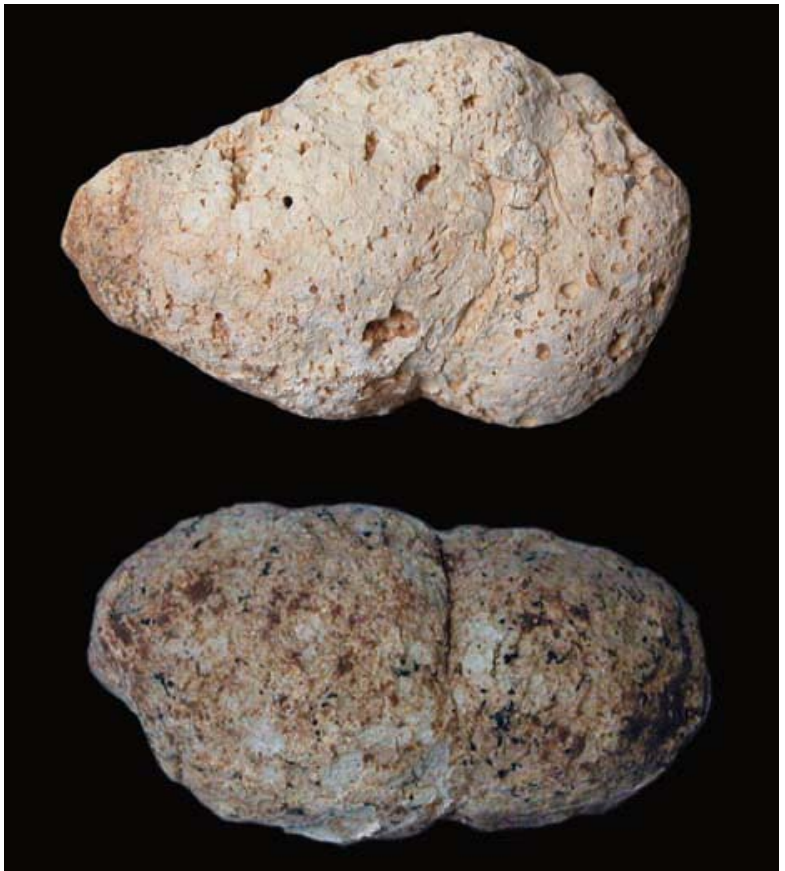

Figure 41. Villacastín. Coprolites of Crocuta crocuta subsp, intermedia from the karstic site of Villacastín, Central System. Photograph: J.S. Carrión.

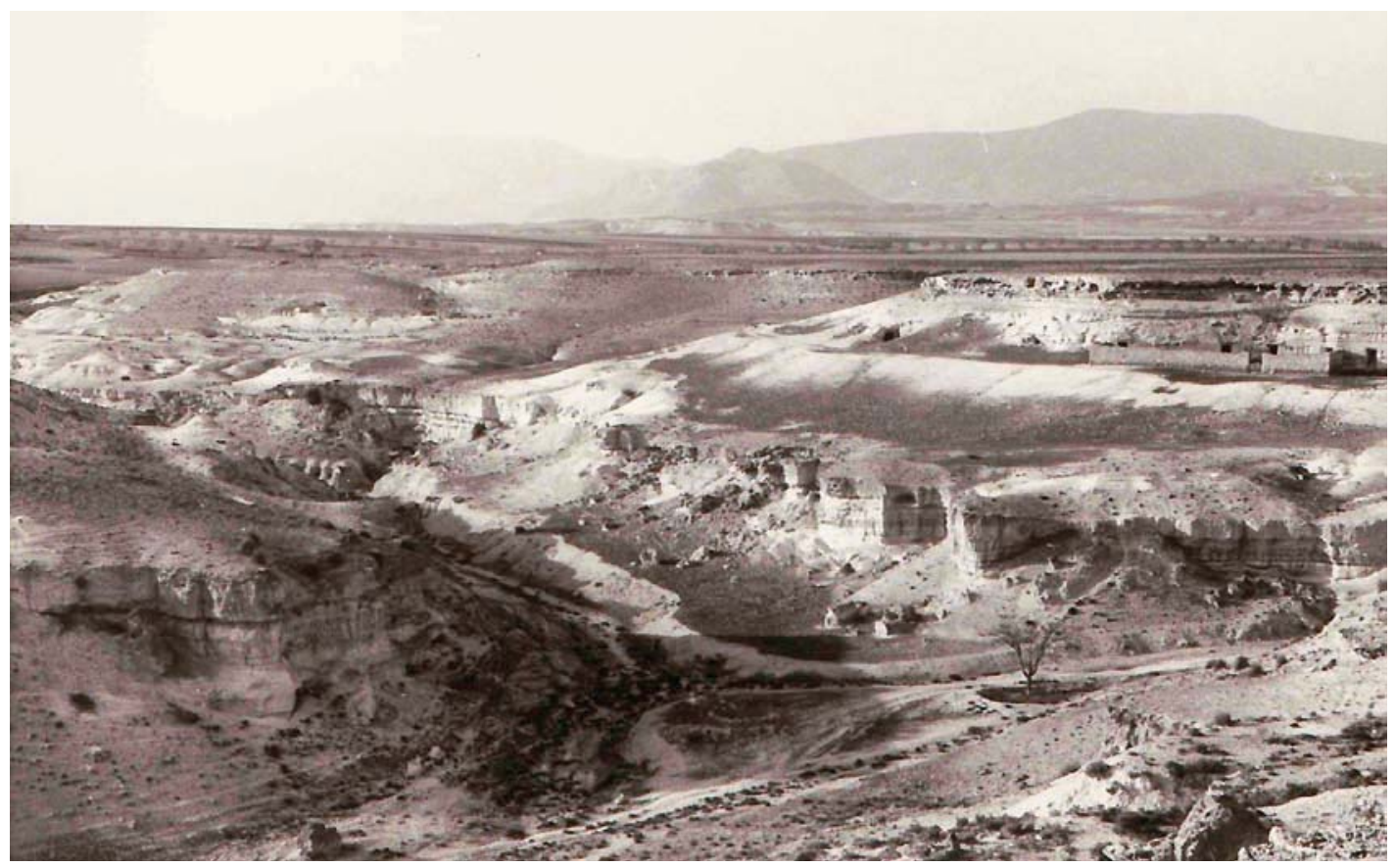

Figure 42. Yeseras. Yeseras, a Lower Pleistocene palaeontological site at the Guadix Basin, Granada. All samples for pollen were sterile. Photograph: S. Leroy. 


\section{Bibliography}

Acosta, P., 1968 La pintura rupestre esquemática hispana, Memorias del Seminario de Prehistoria y Arqueología 1, Salamanca.

Agustí, B., Alcalde G., Burjachs F., Buxó R., Juan-Muns N., Oller J., Ros M.T. and Rueda J.M., 1987 'El yacimiento del Paleolítico Medio de la Cueva 120 (La Garrotxa, Cataluña). Primeros resultados', Cuaternario y Geomorfología 1, 1-13.

Agustí, J. and Julià, R., 1990 'Palaeoclimatic inferences from the Plio-Pleistocene continental sequence of the Guadix-Baza basin (Spain)', Paleobiologie Continentale 17, 269-79.

Aira-Rodríguez, M.J., Saa-Otero, P. and Taboada-Castro, T., 1988 Estudios paleobotánicos y edafológicos en yacimientos arqueológicos de Galicia, Dirección General de Patrimonio Histórico, Coruña.

Alcover, J.A., Pérez-Obiol, R., Yll, E.I. and Bover, P., 1999 'The diet of Myotragus balearicus Bate 1909 (Artiodactyla, Caprinae), an extinct bovid from the Balearic Islands, evidence from coprolites', Biological Journal of The Linnean Society 66, 57-74.

Alday, A., 1995 'Los elementos de adorno personal de la Cueva del Moro de Olvena y sus derivaciones cronológico-culturales', Bolskan: Revista de arqueología del Instituto de Estudios Altoaragoneses 12, 193-214.

Allué, E., Burjachs, F., Giralt, S., Vallverdú J. and Julià, R., 1998 'Paleoenvironments from a middle palaeolithic site (Abric Romaní, Barcelona, Spain)', Proceedings of XIII UISPP Congress 2, 347-354.

Allué, E. and Renault-Miskovsky, J., 1999 'Palynological analysis of Els Vinyets and La Cativera (El Catllar, Spain)', Arkeos 5, 101-109.

Alonso, M., 1998 'Las lagunas de la España peninsular', Limnetica 15, 1-176.

Altuna, J., Baldeón, A. and Mariezkurrena, K., 1985 Cazadores magdalenienses en la cueva de Erralla, Munibe 37.

Altuna, J., Baldeón, A. and Mariezkurrena, K., 1990 La cueva de Amalda (Zestoa, País Vasco). Ocupaciones Paleolíticas y Postpaleolíticas, Sociedad de Estudios Vascos 4, Spain.

Altuna, J. and Merino, J.M., 1984 El yacimiento prehistórico de la cueva de Ekain (Deba, Gipuzkoa), Sociedad de Estudios Vascos 1, Spain.

Arlegui, M., 1992 'El yacimiento celtibérico de Castilmontán, Somaén (Soria), El sistema defensivo’, II Symposium de Arqueología Soriana, Soria 1989, 495-513.
Arnáiz, M.A., 1990 ‘Las ocupaciones de San Quirce del río Pisuerga: reflexiones sobre la utilización del espacio y sus implicaciones', Boletín del Seminario de Estudios de Arte y Arqueología 56, 25-37.

Arribas, A., Aura, E., Carrión, J., Jordá, J. and Pérez Ripoll, M., 2004a 'Presencia de hiena manchada en los depósitos basales (Pleistoceno Superior final) del yacimiento arqueológico de la Cueva de Nerja (Málaga, España)', Revista Española de Paleontología 19, 109-121.

Arribas, A., Baeza, E., Bermúdez, D., Blanco, S., Durán, J.J., Garrido, G., Gumiel, J.C., Hernández, R., Soria, J.M. and Viseras, C., 2004b 'Nuevos registros paleontológicos de grandes mamíferos en la Cuenca de Guadix-Baza (Granada), aportaciones del Proyecto Fonelas al conocimiento sobre las faunas continentales del Plioceno-Pleistoceno europeo', Boletín Geológico y Minero 115, 567-581.

Arribas, A. and Palmqvist, P., 1998 'Taphonomy and paleoecology of an assemblage of large mammals: hyaenid activity in the Lower Pleistocene site at Venta Micena (Orce, Guadix-Baza Basin, Granada, Spain)', Geobios 31, 3-47.

Asquerino, M.D. and López-García, P., 1981 'La Cueva del Nacimiento (Pontones), un yacimiento Neolítico en la Sierra del Segura', Trabajos de Prehistoria 38, 109-152.

Badal, E. and Carrión, Y., 2001 'Del glaciar al interglaciar, Los paisajes vegetales a partir de los restos carbonizados hallados en las cuevas de Alicante' in V. Villaverde (ed) De Neandertales a cromañones. El inicio del poblamiento humano en las tierras valencianas, Universitat de Valencia, Valencia. 21-44.

Badal, E., Soler, B., Villaverde, V. and Aura, E., 1990 'Nota sobre un hogar solútero-gravetiense del Abric de la Ratlla del Bubo (Crevillent, Alicante)', Archivo de Prehistoria Levantina 20, 79-93.

Barber, K. and Charman, D., 2003 'Holocene palaeoclimate records from peatlands', in A. Mackay, R. Battarbee, J. Birks and F. Oldfield (eds), Global Change in the Holocene, Arnold, London. 210-26.

Bennett, K.D. and Willis, K.J., 2001 'Pollen', in J.P. Smol, H.J.B. Birks and W.M. Last (eds), Tracking Environmental Change using Lake Sediments Volume 3. Terrestrial, algal and siliceous indicators. Kluwer Academic Publications, Dordrecht. 5-32.

Bernaldo de Quirós, F. and Cabrera, V., 2000 'Excavaciones arqueológicas en la cueva de El Castillo, Puente Viesgo, 1980-1999', in R. Ontañón, Actuaciones Arqueológicas en Cantabria 1984-1999, Gobierno de Cantabria, Santander. 23-34. 
Betancourt, J.L., 2004 'Arid lands paleobiogeography: The fossil rodent midden record in the Americas', in M.V. Lomolino and L.R. Heaney (eds), Frontiers in Biogeography, New Directions in the Geography of Nature, Sinauer Associates Inc. 27-46.

Birks, J.H.B., 1986 'Late Quaternary biotic changes in terrestrial and lacustrine environments, with particular reference to north-west Europe', in B.E. Berglund (ed), Handbook of Holocene Palaeoecology and Palaeohydrology, Wiley, Chichester. 3-65.

Birks, J.H.B. and Birks, H.H., 1980 Quaternary Palaeoecology. University Park Press, Baltimore.

Bonet, H., Mata, C., Sarrión, I., Dupré, M. and Renault-Miskovsky, J., 1981 El poblado ibérico del Puntual dels Llops (El Colmenar), (Olocau, Valencia), Servicio de Investigación Prehistórica, Serie de Trabajos Varios 71, Diputacion Provincial de Valencia.

Botella, D., Barroso, C., Riquelme, J.A., Abdessadok, S., Caparrós, M., Verdú, L., Monge, G. and García, J.A., 2006 'La Cueva del Ángel (Lucena, Córdoba), un yacimiento del Pleistoceno medio y Superior del sur de la Península Ibérica', Trabajos de Prehistoria 2, 153-65.

Bottema, S. and Woldring, H., 1994 'Bronze age and Byzantine pollen of the Kestel tin-mine (Turkey) and its possible origin, practical and experimental pollen analysis in archaeological context', in O.K. Davis (ed), Aspects of Archaeological Palynology, Methodology and Applications, AASP Contribution Series 29. 7-15.

Boyer-Klein, A., 1981 'Análisis palinológico del Rascaño’ in J. González Echegaray and I Barandiaran Maestu (eds) El Paleol tico Superior de la Cueva del Rascaño (Santander), Centro de Investigación y Museo de Altamira, Santander. 216-20.

Boyer-Klein, A., 1988 'Analyses polliniques au Tardiglaciaire dans le Nord de l'Espagne, au sujet du Dryas I, II, III', in J. Civis and M.F. Valle (eds), Actas del VI Simposio de Palinología, APLE, Salamanca. 277-85.

Boyer-Klein, A., 1989 'Análisis polínico de la cueva de Zatoya', in I. Barandiarán and A. Cava (eds), El yacimiento prehistórico de Zatoya (Navarra), Suplementos de Trabajos de Arqueología 8, Pamplona. 231-35.

Bryant, V.M. and Holloway, R., 1983 'The role of palynology in archaeology', Advances in Archaeological Method and Theory 6, 191-224.

Burjachs, F., 1988a ‘Análisis polínico de los niveles cerámicos de la Cova 120 (Alta Garrotxa, Catalunya)', Acta Salmanticensia 65, 285-90.
Burjachs, F., 1988b Informe de les anàlisis pollíniques efectuades amb sediments de les Sitges de la UAB i del poblat ibèric de Can Olivé (Cerdanyola del Vallès, Vallès Occidental), Centre de Recerques Arqueològiques de Cerdanyola, Cerdanyola del Vallès.

Burjachs, F., 1990 Informe de l'anàlisi palinològica realitzada amb sediments de la campanya de 1989 de la Font del Ros (Berga, Bergadà), University Autonomous of Barcelona, unpublished report.

Burjachs, F., 1991a 'El paisatge de la vall durant l'ocupació del Paleolític Mitjà. Anàlisi pol-línica', Cypsela 9, J. Agustí et al. (eds) La Cova 120, parada de caçadors-recolectors del Paleolític Mitjà. 7-20.

Burjachs, F., 1991b Informe de los análisis palinológicos del poblado de Los Millares (Almería), University of Granada, unpublished report.

Burjachs, F., 1994 'Palynology of the Upper Pleistocene and Holocene of the North-East Iberian Peninsula, Pla de l'Estany', Historical Biology 9, 17-33.

Burjachs, F., 1999 'Análisis polínico de los sectores 1 y 2', in C. Olaria (ed), Cova Matutano (Vilafamés, Plana Alta, Castelló). Un modelo ocupacional del magdaleniense superior-final en la vertiente mediterránea peninsular, Servei d'Investigacions Arqueològiques i Prehistòriques, Castellón. 247-253.

Burjachs, F., 2000a 'Informe palinològic del jaciment de Cal Guardiola (Terrassa, Vallès Occidental)', Barcelona. Servei d'Arqueologia, Generalitat de Catalunya, Barcelona.

Burjachs, F., 2000b 'Informe palinològic del jaciment de La Prunera (Olot, La Garrotxa)'. Universitat Autònoma de Barcelona, unpublished report.

Burjachs, F., 2001 'Paleoecología del Homo antecessor, Palinología de las unidades TD5, 6 y 7 de la "Gran Dolina" de Atapuerca (Burgos, Spain)', in S. Moreno Grau, B. Elvira Rendueles and J.M. Moreno Angosto (eds), XIII Simposio de la Asociación de Palinólogos en Lengua Española. ( $A P L E)$, Libro de textos completos, Universidad Politécnica de Cartagena, Servicio de Publicaciones, Cartagena. 365-72.

Burjachs, F., 2002 'Palinología', in E. Carbonell (ed), Abric Romaní, nivel I. Models d'ocupació de curta durada de fa 46.000 anys a la cinglera del Capelló (Capellades, Anoia, Barcelona), Universitat Rovira i Virgili, Tarragona. 323.

Burjachs, F., in press Arqueobotànica del jaciment 'Sitges de la $U A B$, Limes, Barcelona. 
Burjachs, F. and Julià, R., 1994 'Abrupt climatic changes during the last glaciation based on pollen analysis of the Abric Romaní, Catalonia, Spain', Quaternary Research 42, 30815.

Burjachs, F. and Julià, R., 1996 'Palaeoenvironmental evolution during the Middle-Upper Palaeolithic transition in the NE of the Iberian Peninsula', in E. Carbonell and M. Vaquero (eds), The Last Neandertals, the first anatomically modern humans, Universitat Rovira i Virgili, Tarragona. 203-18.

Burjachs, F. and Pérez-Obiol, R., 1988 'Informe de l'anàlisi paleopollínica del sediments arqueològics de la Bòbila Madurell (Sant Quirze del Vallès, Vallès Occidental)'. Servei d'Arqueologia de la Generalitat de Catalunya, unpublished report.

Burjachs, F. and Pérez-Obiol, R., 1989 'Informe de l'anàlisi paleopollínica dels sediments arqueològics de l'abric de la Font Voltada (Montbrió de la Marca, Conca de Barberà)', unpublished report.

Burjachs, F., Rodó, X. and Comín, F.A., 1996 'Gallocanta, ejemplo de secuencia palinológica en una laguna efímera', in B. Ruiz Zapata (ed), Estudios palinológicos, Servicio de Publicaciones de la Universidad de Alcalá, Alcalá de Henares. 25-29.

Burjachs, F., Giralt, S., Roca, J.R., Seret, G. and Julià, R., 1997 'Palinología holocénica y desertización en el Mediterráneo occidental', in J.J. Ibáñez, B.L. Valero and C. Machado, C. (eds), El paisaje mediterráneo a través del espacio y del tiempo. Implicaciones en la desertificación, Geoforma Editores, Logroño. 379-94.

Burjachs, F., Febrero, A., Rodríguez-Ariza, M.O., Buxó, R., Araus, J.L. and Julià, R., 2000 'Holocene pollen sequences and carbon isotope discrimination of plant remains in Spain, evidence of a progressive increase in aridity', in P. Balabanis, D. Peter, A. Ghazi and M. Tsogas (eds), Mediterranean Desertification. Research Results and policy implications. Directorate General Research, Brussels. 11-20.

Burjachs, F., López-Sáez, J.A. and Iriarte, M.J., 2003 'Metodología arqueopalinológica', in R. Buxó and R. Piqué (eds), La recogida de muestras en arqueobotànica: objetivos y propuestas metodológicas. Museu d'Arqueologia de Catalunya, Barcelona. 11-18.

Burney, D.A. and Burney, L.P. 1993 'Modern pollen deposition in cave sites, experimental results from New York State', New Phytologist 124, 523-35.

Burney, D.A., Brook, G.A. and Coward, J.B., 1994 'A Holocene pollen record for the Kalahari Desert of Botswana for a U-series dated speleothem', The Holocene 4, 225-32.
Butzer, K.W. , Butzer, E.K. and Mateu, J.F., 1986 'Medieval Muslim communities of the Sierra de Espadán, Kingdom of Valencia', Viator, Medieval and Renaissance Studies 17, 339-413.

Cacho, C., Fumanal, M.P., López-García, P., López-Sáez, J.A., Pérez Ripoll, M., Mártínez Valle, R., Uzquiano, P., Arnanz, A.M., Sánchez Marco, A., Sevilla, P., Morales Muñiz, A., Roselló, E., Garralda, M.D. and García-Carrillo, M., 1995 'El Tossal de la Roca (Vall D’Alcalà, Alicante) Reconstrucción paleoambiental y cultural de la transición del Tardiglaciar al Holoceno inicial', Recerques del Museu D'Alcoy 4, 11.

Cacho, C., Ripoll, S., Jordá, J., Muñoz, F., Yravedra, J. and Maicas, R., 2003 'Ocupaciones magdalenienses en la Meseta Norte, La Peña de Estebanvela (Segovia)', Zephyrus 56, 19-37.

Calaforra, J.M. and Pulido-Bosch, A., 2003 'Evolution of the gypsum karst of Sorbas (SE Spain)', Geomorphology 50, 173-80.

Campy, M. and Chaline, J., 1993 'Missing records and depositional breaks in French Late Pleistocene cave sediments', Quaternary Research 40, 318-31.

Carrión, J.S., 1992a 'Late Quaternary pollen sequence from Carihuela Cave, southeastern Spain', Review of Palaeobotany and Palynology 71, 37-77.

Carrión, J.S., 1992b 'A palaeoecological study in the western Mediterranean area. The Upper Pleistocene pollen record from Cova Beneito (Alicante, Spain)', Palaeogeography, Palaeoclimatology, Palaeoecology 92, 1-14.

Carrión, J.S., 2002a 'Patterns and processes of Late Quaternary environmental change in a montane region of southwestern Europe', Quaternary Science Reviews 21, 2047-66.

Carrión, J.S., 2002b 'A taphonomic study of modern pollen assemblages from dung and surface sediments in arid environments of Spain', Review of Palaeobotany and Palynology 120, 217-32.

Carrión, J.S. and Dupré, M., 1996 'Late Quaternary vegetational history at Navarrés, eastern Spain. A two core approach', New Phytologist 134, 177-91.

Carrión, J.S. and Dupré, M., 2002 'Los paisajes vegetales de Altamira en el Paleolítico Superior', in J.A. Lasheras Corruchaga (ed), Redescubrir Altamira, Turner, Madrid. 141-50.

Carrión, J.S. and Munuera, M., 1997 'Upper Pleistocene palaeoenvironmental change in eastern Spain: new pollen analytical data from Cova Beneito (Alicante)', Palaeogeography, Palaeoclimatology, Palaeoecology 128, $287-$ 99. 
Carrión, J.S. and Scott, L., 1999 'The challenge of pollen analysis in palaeoenvironment studies of hominid beds, the record from Sterkfontein caves', Journal of Human Evolution 36, 401-8.

Carrión, J.S. and van Geel, B., 1999 'Fine-resolution Upper Weichselian and Holocene palynological record from Navarrés (Valencia, Spain) and a discussion about factors of Mediterranean forest succession', Review of Palaeobotany and Palynology 106, 209-36.

Carrión, J.S., Munuera, M. and Navarro, C., 1998 'The palaeoenvironment of Carihuela Cave (Granada, Spain), a reconstruction on the basis of palynological investigations of cave sediments', Review of Palaeobotany and Palynology 99, 317-40.

Carrión, J.S., Munuera, M., Navarro, C., Burjachs, F., Dupré, M. and Walker, M.J., 1999a 'The palaeoecological potential of pollen records in caves, the case of Mediterranean Spain', Quaternary Science Reviews 18, 1061-73.

Carrión, J.S., Scott, L. and Vogel, J. 1999b 'Twentieth-century changes in montane vegetation in the eastern Free State, South Africa, derived from the analysis of hyrax dung middens', Journal of Quaternary Science 14, 1-16.

Carrión, J.S., Munuera, M., Navarro, C., and Sáez, F., 2000a 'Paleoclimas e historia de la vegetación cuaternaria en España a través del análisis polínico. Viejas falacias y nuevos paradigmas', Complutum 11, 115-42.

Carrión, J.S., Scott, L., Huffman, T. and Dreyer, C., 2000b 'Pollen analysis of Iron Age cow dung in southern Africa', Vegetation History and Archaeobotany 9, 239-49.

Carrión, J.S., Brink, J., Scott, L. and Binneman, J., 2000c 'Palynology of hyena coprolites from Oyster Bay, southeastern Cape coast, South Africa, the palaeo-environment of an open-air Howieson's Poort occurrence', South African Journal of Science 96, 449-53.

Carrión, J.S., Riquelme, J.A, Navarro, C. and Munuera, M., 2001a 'Pollen in hyaena coprolites reflects late glacial landscape in southern Spain', Palaeogeography, Palaeoclimatology, Palaeoecology 176, 193-205.

Carrión, J.S., Andrade, A., Bennett, K.D., Munuera, M. and Navarro, C., 2001b 'Crossing forest thresholds. Inertia and collapse in a Holocene sequence from south-central Spain', The Holocene 11, 635-53.

Carrión, J.S., Munuera, M., Dupré, M. and Andrade, A., 2001c 'Abrupt vegetation changes in the Segura mountains of southern Spain throughout the Holocene', Journal of Ecology 89, 783-97.

Carrión, J.S., Sánchez-Gómez, P., Mota, J.F., Y1l, E.I. and
Chaín, C., 2003a 'Fire and grazing are contingent on the Holocene vegetation dynamics of Sierra de Gádor, southern Spain', The Holocene 13, 839-49.

Carrión, J., Y1l, E., Walker, M., Legaz, A., Chaíns, C. and López, A., 2003b 'Glacial refugia of temperate, Mediterranean and Ibero-North African flora in south-eastern Spain: new evidence from cave pollen at two Neandertal man sites', Global Ecology and Biogeography 12, 119-29.

Carrión, J.S., Y11, R., González-Sampériz, P. and Scott, L., 2004a 'Advances in the palynology of cave sites from Spain, taphonomical and palaeoecological aspects' in B. Andreo and J.J. Durán (eds) Investigaciones en sistemas kársticos españoles. Instituto Geológico y Minero de España, Madrid, Serie Hidrogeología y Aguas Subterránea 12. 351-66

Carrión, J.S., Willis, K.J. and Sánchez Gómez, P., 2004b 'Holocene forest history of the eastern plateaux in the Segura Mountains (Murcia, southeastern Spain)', Review of Palaeobotany and Palynology 132, 219-36.

Carrión, J.S., Yll, E.I. and Riquelme, J.A., 2004c 'Perspectivas del análisis polínico de coprolitos y otros depósitos biogénicos útiles en la inferencia paleoambiental', in S. Rubio Jara and E. Baquedano Pérez (eds) Miscelánea en Homenaje a Emiliano Aguirre, Paleontología. Museo Arqueológico Regional, Madrid, 128-39.

Carrión, J.S., Scott, L. and Marais, E., 2006b 'Environmental implications of pollen spectra in bat droppings from southeastern Spain and potential for palaeoenvironmental reconstructions', Review of Palaeobotany and Palynology 140, $175-86$.

Carrión, J.S., Scott, L., Arribas, A., Fuentes, N., Gil, G. and Montoya, E., 2007a 'Pleistocene landscapes in central Iberia inferred from pollen analysis of hyena coprolites', Journal of Quaternary Science 22, 191-202.

Carrión, J.S., Fuentes, N., Gonzalez-Samperiz, P., Quirante, L.S., Finlayson, J.C., Fernandez, S. and Andrade, A., $2007 b$ 'Holocene environmental change in a montane region of southern Europe with a long history of human settlement', Quaternary Science Reviews 26, 1455-75.

Carrión, J.S., Finlayson, C., Finlayson, G., Allué, E., LópezSáez, J.A., López-García, P., Fernández, S., Gil, G., Fuentes, N. and González-Sampériz, P., 2008 'A coastal reservoir of biodiversity for Upper Pleistocene human populations. Palaeoecological investigations in Gorham's Cave (Gibraltar) in the context of the Iberian Peninsula', Quaternary Science Reviews 27, 2118-35.

Casado, S. and Montes, C. 1995 Guía de los lagos y humedales de España. JM Reyero, Madrid. 
Caseldine, C.J., McGarry, S., Baker, A., Hawkesworth, C. and Smart, P.L., 2007 'Late Quaternary speleothem pollen in the British Isles', Journal of Quaternary Science 23, 193 200 .

Castán, A. and Baldellou, V., 1985 'Excavaciones en la cueva de Chaves de Bastaras (Casbas-Huesca)', Bolskan: Revista de Arqueología del Instituto de Estudios Altoaragoneses 1, 9-38.

Castillo, J., Alvarado, M., Alonso, A., Molano, J., Fernández, J. and Cerrillo, E., 1994 'Cáparra, las relaciones comerciales a través de su aprovisionamiento cerámico’ in Dupré, X. (ed) La ciutat en el món romà, Tarragona. 21-24.

Castro, P.V., Chapman, R.W., Suriñach, S., Lull, V., Micó, R., Rihuete, C., Risch, R. and Sanahuja, M.E., 1999 Proyecto Gatas. 2. La dinámica arqueoecológica de la ocupación prehistórica. Arqueología, Monografías, Junta de Andalucía, Sevilla.

Cattani, L., Garcia Antón, M. and Renault-Miskovsky, J., 1994 'The lower and middle Pleistocene in the circum mediterranean region. Attempts and results of archaeo palynological correlations', AASP Contributions Series 29, 27-52.

Cebrià, A., Esteban Amat, A., Parra, I. and Yll, E.I., 1988 'Análisis polínico de una secuencia estratigráfica y de un coprolito', in Olaria, C. (ed) Cova Fosca. Un asentamiento neolítico de cazadores y pastores en la serranía del Alto Maestrazgo, Diputación de Castellón, Castellón. 257-64.

Cirujano, S., 1990 Flora y vegetación de las lagunas y humedales de la provincia de Albacete, Instituto de Estudios Albacetenses, Albacete.

Coles, G.M. and Gilbertson, D.D., 1994 'The airfall-pollen budget of archaeologically important sites, Creswell Crags, England', Journal of Archaeological Science 21, 735-55.

Coles, G.M., Gilbertson, D.D., Hunt, C.O. and Jenkinson, R.D.S., 1989 'Taphonomy and the palynology of cave sediments', Cave Science 16, 83-89.

Consuegra, S., Gallego, M.M. and Castañeda, N., 2004 'Minería neolítica de sílex de Casa Montero (Vicálvaro, Madrid)', Trabajos de Prehistoria 61, 127-40.

Contreras, F., Nocete, F. and Sánchez, M., 1985 'Análisis histórico de las comunidades de la Edad del Bronce de la Depresión Linares-Bailén y estribaciones meridionales de Sierra Morena. Sondeo estratigráfico en el Cerro de Plaza de Armas de Sevilleja (Espelúy, Jaén)', Anuario Arqueológico de Andalucía 2, 141-49.

Cortés, M. and Sanchidrián, J.L., 1999 'Dinámica cultural del Pleistoceno Superior en la costa de Málaga', Cuaternario y Geomorfología 13, 63-77.
Coûteaux, M., 1977 ‘A propos de l'interprétation des analyses polliniques de sédiments minéraux, principalement archéologiques', in H. Laville and J. Renault-Miskovsky (eds), Approche écologique de l'homme fossile, Supplément du Bulletin de l'Association Française pour l'Etude du Quaternaire 47. 259-76.

Cruz, D.J., 1991 A Mamoa 1 de Chã de Carvalhal no contexto arqueológico da Serra da Aboboreira e da Pré-História recente do Norte de Portugal, Coimbra. University of Coimbra, Portugal.

Cuchí, J.A. and Sancho, C., 1995 'Marco geológico y geomorfológico de la Cueva del Moro de Olvena (Huesca)', Bolskan 12, 19-28.

Davis, B.A.S., 1994 Paleolimnology of Holocene environmental change from endorheic lakes in the Ebro basin, NorthEast Spain, PhD Thesis, University of Newcastle-upon-Tyne.

Davis, O.K., 1990 'Caves as sources of biotic remains in arid western North America', Palaeogeography, Palaeoclimatology, Palaeoecology 76, 331-48.

Davis, O.K., 1998 'Palynological evidence for vegetation cycles in a 1.5 million year pollen record from the Great Salt Lake, Utah, USA', Palaeogeography, Palaeoclimatology, Palaeoecology 138, 175-85.

Davis, O.K. and Anderson, R.S., 1987 'Pollen in packrat (Neotoma) middens, pollen transport and the relationship of pollen to vegetation', Palynology 11, 185-98.

Davis, O.K. and Mariscal, B., 1994 'A comparison of archaeological palynology of Almería, Spain, and coastal southern California, USA', in O.K. Davis (ed), Aspects of Archaeological Palynology, Methodology and Applications. AASP Contributions Series 29, 75-82.

Delibes, G. and Moure, A., 1973 'Excavaciones arqueológicas en la villa romana de Almenara de Adaja (Provincia de Valladolid). Campaña de 1969', Noticiario Arqueológico Hispánico, Arqueología 2, 9-50.

Díez, C., García, M.A., Gil, E., Jordá, J.F., Ortega, A.I., Sánchez, A. and Sánchez, B., 1989 'La Cueva de Valdegoba (Burgos). Primera campaña de excavaciones', Zephyrus 42, 55-74.

Dimbleby, G.W., 1985 The Palynology of Archaeological Sites. Academic Press, London.

Dricot, L. and Leroy, S., 1989 'Peptization and sieving for palynological purposes', Geobound 2, 114-26. 
Dorado-Valiño, M., Valdeolmillos, A., Ruiz-Zapata, M.B., GilGarcía, M.J. and de Bustamante, I., 2002 'Climatic changes since the lateglacial-holocene transition in La Mancha Plain (south-central Iberian Peninsula, Spain) and their incidence on Las Tablas de Daimiel marshlands', Quaternary International 94, 73-84.

Dueñas, M.A. and Recio, J.M., 2000 Bases ecológicas para la restauración de los humedales de La Janda (Cádiz, España). Servicio de Publicaciones de la Universidad de Córdoba, Córdoba.

Dupré, M., 1988 Palinología y paleoambiente. Nuevos datos españoles. Referencias, Serie de Trabajos Varios 84, Servicio de Investigación Prehistórica, Diputación Provincial de Valencia.

Dupré, M. and Renault-Miskovsky, J., 1990 'El hombre y su impacto en las zonas bajas mediterráneas. Datos palinológicos de sedimentos arqueológicos holocenos', Archivo de Prehistoria Levantina 20, 133-41.

Dupré, M., Fumanal, M.P., Robls, R., Acuña, J.P. and Martí, B., 1983 'Cova de l'Or (Beniarres, Alicante). Campañas de excavación 1975-1979’, Noticiario arqueológico hispano 16, 9-56.

Dupré, M., Fumanal, M.P. and La Roca, N., 1985 'Modifications de I'environnement endoréique. La tourbière de la Canal de Navarrés (Valencia, Espagne)', Cahiers Ligures de Préhistoire et de Protohistoire 2, 297-311.

Dupré, M., Pérez-Obiol, R. and Roure, J.M., 1994 ‘Análisis polínico del sondeo TU de la turbera de Torreblanca (Castellón, España)', in M. Dupré, M.E. Burgaz, I. Mateu and J. Güemes (eds), X Simposio de Palinología (APLE), Valencia. 165-74.

Dupré, M., Fumanal, M.P., Martínez Gallego, J., Pérez Obiol, R., Roure, J.M. and Usera, J., 1996 'The Laguna de San Benito (Valencia, Spain), palaeoenvironmental reconstruction of an endorheic system', Quaternaire 7, 177-86.

Dupré, M., Carrión, M., Fumanal, M.P., La Roca, N., Martínez, J. and Usera, J., 1998a 'Evolution and palaeoenvironmental conditions of an interfan area in eastern Spain (Navarrés, Valencia)', Il Quaternario 11, 95-105.

Dupré, M., Fumanal, M.P., Sanjaume, E., Santisteban, C., Usera, J. and Viñals, M.J., 1998b 'Quaternary evolution of the Pego coastal lagoon (Southern Valencia, Spain)', Palaeogeography, Palaeoclimatology, Palaeoecology $\mathbf{6 8}$, 291-99.

Dupré, M., Ferrer, C.,González-Sampériz, P., Roca de Togores, C., Grau, E., Máñez, S. and Soler, J.A., 1999 'Cova d'en Pardo, Planes, Alicante: primeros resultados de una investigación pluridisciplinar en un yacimiento prehistórico', in Universidad de Valencia (ed) Geoarqueología quaternari litoral: memorial María Pilar Fumanal. 269-82.
Expósito, I. and Burjachs, F., 2007 'Informe d'un test d'anàlisi palinològica de tres mostre sedimentàries procedents de les Illes Columbrets (Castelló, País Valencià)', Universitat de València, unpublished report.

Faegri, K and Iversen, J., 1975 Textbook of Pollen Analysis. Halfner Press, New York.

Fernández-Cortés, A., Calaforra, J.M., Jiménez-Espinosa, R. and Sánchez-Marto, F., 2006 'Geostatistical spatiotemporal analysis of air temperature as an aid to delineating thermal stability zones in a potential show cave. Implications for environmental management', Journal of Environmental Management 81, 371-83.

Fernández, S., Carrión, J.S., Fuentes, N., González-Sampériz, P., Gil, G., García-Martínez, M.S., Vega-Toscano, L.G. and Riquelme, J.A., 2007 'Palynology of Carihuela Cave, southern Spain: completing the record', Geobios 40, 7590 .

Fernández-Peris, J., 2004 'Datos sobre la incidencia de carnívoros en La Cova de Bolomor (Tavernes de la Valldigna, Valencia)', in E. Baquedano and S. Rubio (eds), Miscelánea en Homenaje a Emiliano Aguirre, Volumen IV, Arqueología. Museo Arqueológico Regional, Valencia. 141-57.

Fernández-Rodríguez, C., Ramil-Rego, P. and Martínez Cortizas, A., 1995 'Characterization and depositional evolution of hyaena (Crocuta crocuta) coprolites from La Valiña Cave (northwest Spain)', Journal of Archaeological Science 22, 597-607.

Fernández-Zamudio, R., Sousa, A. and García-Murillo, P., 2007 Laguna de las Madres. Junta de Andalucía, Sevilla.

Florschütz, F., Menéndez-Amor, J. and Wijmstra, T., 1971 'Palynology of a thick Quaternary succession in S. Spain', Palaeogeography, Palaeoclimatology, Palaeoecology 10, 233-64.

Ford, T.D. and Pedley, H.M. 1996 'A review of tufa and travertine deposits of the world', Earth Science Reviews 41, 117-75.

Fortea, F.J., 1990 'Abrigo de La Viña: informe de las campañas 1980-1986' in Excavaciones arqueológicas en Asturias: 1983-86. 55-68.

Fortea, F.J. and Jordá, F., 1976 'La cueva de Les Malladetes y los problemas del Paleolítico Superior del Mediterráneo español', Zephyrus 16-17, 129-66.

Fuentes, N., García Martínez, M., González Sampériz, P., Fernández, S., Carrión, J.S., Ros, M., López Campuzano, M. and Medina, J., 2005 'Degradación ecológica y cambio cultural durante los últimos cuatro mil años en el sureste ibérico semiárido’, Anales de Biología 27, 69-84. 
Fuentes, N., Carrión, J.S., Fernández, S., Nocete, F., Lizcano, R. and Pérez Bareas, C., 2007 'Análisis polínico de los yacimientos arqueológicos Cerro del Alcázar de Baeza y Eras del Alcázar de Úbeda (Jaén)', Anales de Biología 29, 85-93.

Fumanal, M.P., 1986 Sedimentología y clima en el País Valenciano. Las cuevas habitadas en el Cuaternario reciente. Diputación Provincial de Valencia, Valencia.

Fumanal, M.P., Hernández, M.S., Ferrer, C., Serna, A., Batlle, J., Martínez, J. and Bordas, V., 1996 'Estudio geoarqueológico de Cabezo Redondo (Villena, Alicante), un yacimiento de la edad de bronce y sus condicionantes medioambientales', Cuaternario y Geomorfología 10, 5.

Galobart, A., Maroto, J., Menéndez, E., Ros, X., Gaete, R. and Colomer, F., 1990 'El yacimiento del Pleistoceno Inferior de Incarcal (Crespià, Girona)' in Reunión de tafonomía y fosilización, Madrid. 161-67.

García-Antón, M., 1987 'Estudio polínico preliminar de los yacimientos de la Sierra de Atapuerca (Burgos)', in E. Aguirre, E. Carbonell and J.M. Bermúdez de Castro (eds), El Hombre fósil de Ibéas y el Pleistoceno de la Sierra de Atapuerca, Publicaciones de la Junta de Castilla y León, Valladolid. 55-59.

García-Antón, M., 1989 Estudio palinológico de los yacimientos mesopleistocenos de Atapuerca (Burgos): reconstrucción paisajística y paleoclimática, $\mathrm{PhD}$ Thesis, Universidad Autónoma de Madrid.

García-Antón, M., 1995 'Pollen analysis of Middle Pleistocene palaeovegetation at Atapuerca', in J.M. Bermúdez, J.L. Arsuaga and E. Carbonell (eds), Human Evolution in Europe and the Atapuerca Evidence, Publicaciones de la Junta de Castilla y León, Valladolid. 147-65.

García-Antón, M. and Casado, M.A., 1994 ‘Aplicación de análisis numéricos para la zonación de diagramas polínicos, yacimiento de 'Galería', Atapuerca (Burgos)', in I. La-Serna (ed), Polen y esporas, contribución a su conocimiento, Publicaciones de la Universidad de la Laguna, La Laguna. 371-79.

García-Antón, M. and Sainz Ollero, H., 1991 'Pollen records on the Atapuerca Middle Pleistocene site (Burgos, Spain)', Palaeogeography, Palaeoclimatology, Palaeoecology 85, 199-206.

García-Antón, M., Morla, C. and Sainz Ollero, H., 1990 'Consideraciones sobre la presencia de algunos vegetales relictos terciarios en la Península Ibérica durante el Cuaternario', Boletín de la Real Sociedad Española de Historia Natural (Sección Biológica) 86, 95-105.

García-Argüelles, P., Nadal i Lorenzo, J. and Fullola i Pericot, J.M., 2005 'El abrigo del Filador (Margalef de Montsant, Tarragona) y su contextualización cultural y cronológica en el Nordeste peninsular', Trabajos de Prehistoria 62, 65-84.
García del Cura, M.A., Pedley, H.M., Ordóñez, S. and González-Martín, J.A., 2000 'Petrology of a barrage tufa system (Pleistocene to recent) in the Ruidera Lakes Natural Park (Central Spain)', Geotemas 1, 359-63.

Geurts, M., 1977 'Premières données à l'étude palynologique des dépôts calcareux quaternaires en Catalogne', Acta Geologica Hispanica 12, 86-89.

Geurts, M., 1979 'Approche palynostratigraphique des dépôts calcareux quatrenaires dans la région de Banyoles-Besalú (Catalogne)', in R. Julià, M.A. Marqués, A., Mir, D. Serrat and F. Gallart (eds), Actas de la IV Reunión del Grupo de Trabajo del Cuaternario, Banyoles. 106-16.

Gibert, J., Martínez, B., Caporicci, C., Jiménez, C., Ferrández, C., Ribot., F., Soria, F., Pérez-Cuadrado, J.L., Arribas, A., Canals, J., García-Targa, J.M., Iglesias, A. and Romero, R., 1988 'Resumen de las investigaciones paleoantropológicas y arqueológicas de Orce (Granada) y Cueva Victoria (Cartagena)', Coloquios de Paleontología 42, 11-60.

Gibert, J., Leakey, M., Ribot, F., Gibert, L., Arribas, A. and Martínez-Navarro, B., 1995 'Presence of the Cercopithecid genus Theropithecus in Cueva Victoria (Murcia, Spain)', Journal of Human Evolution 28, 31-46.

Gil-García, M.J., Las Heras, R., Núñez, E. and Martínez, J., 1995 'Análisis polínico de un depósito higroturboso de la Sierra de Cameros (La Rioja, España)', Zubia 13, 33-41.

Gil-Romera, G., Scott, L. Marais E. and Brook, G.A., 2007 'Late Holocene environmental change in the northwestern Namib Desert margin, New fossil pollen evidence from hyrax middens', Palaeogeography, Palaeoclimatology, Palaeoecology 249, 1-17.

Giralt, S., Burjachs, F., Roca, J.R. and Julià, R., 1999 'Late Glacial to Early Holocene environmental adjustment in the Mediterranean semi-arid zone of the Salines playa-lake (Alacant, Spain)', Journal of Palaeolimnology 21, 44960 .

Girard, M., 1975 'Prélèvements d'échantillons en grotte et station de terrain sec en vue de l'analyse pollinique', Bulletin de la Societé Préhistorique Française 72, 158-60.

Girard, M. and Renault-Miskovsky, J., 1969 'Nouvelles techniques de préparation en palynologie appliquées à trois sédiments du Quaternaire final de l'Abri Corneille (IstresBouches-du-Rhone)', Bulletin de l'Association Française pour l'Etude du Quaternaire 4, 275-84.

Goeury, C. and de Beaulieu, J.-L., 1979 'A propos de la concentration du pollen à l'aide de la liqueur de Thoulet dans les sédiments minéraux', Pollen et Spores 21, 239-51. 
Gómez-Ferreras, C., López, P. and López Sáez, J.A., ‘1996 Dinámica de la vegetación de las lagunas de Villafáfila (Zamora) durante el Holoceno reciente' in Ruiz Zapata, B. (ed) Estudios Palinológicos, Universidad de Alcalá de Henares, Alcalá de Henares. 57-61.

Gómez-Orellana, L., Ramil-Rego, P. and Muñoz-Sobrino, C., 2007 'The Würm in NW Iberia, a pollen record from Area Longa (Galicia)', Quaternary Research 67, 438-52.

González-Beserán, J.L, González, L.J. and Mujeriego, F.L., 1991 Introducción a la ecología de la Laguna de los Ojos de Villaverde, Instituto de Estudios Albacetenses, Albacete.

González-Morales, M.R., 1990 'El abrigo de Entrefoces (19801983)', in Excavaciones arqueológicas en Asturias 198386. 29-36.

González-Morales, M.R., Ortea Rato, J.A., Díaz González, T.E., Márquez Uría, M.C. and Volman, K.C., 1980 'El conchero asturiense de la cueva de Mazaculos II (La Franca, Asturias), campañas de 1976 a 1978', Noticiario Arqueológico Hispano 9, 35-64.

González-Sampériz, P., 1998 'Estudio palinológico de la Cueva de En Pardo (Planes, Alicante). Primeros resultados', Cuaternario y Geomorfología 12, 45-61.

González-Sampériz, P., 1999 'Análisis de polen subactual en la Cueva de En Pardo (Planes, Alicante), in L. Buxó and C. Pau (eds), Avances en el estudio del Cuaternario español, Girona. 307-16.

González-Sampériz, P., 2001 Análisis palinológico aplicado a la reconstrucción paleoclimática en medios mediterráneos y eurosiberianos. PhD Thesis, Universidad de Zaragoza.

González-Sampériz, P., 2004a Evolución paleoambiental del sector central de la cuenca del Ebro durante el Pleistoceno superior y Holoceno. Instituto Pirenaico de Ecología-CSIC, Zaragoza.

González-Sampériz, P., 2004b 'Análisis palinológico del yacimiento de Los Baños de Ariño (Teruel)' in Utrilla, P., Rodanés, J.M. (eds) El yacimiento arqueológico de Los Baños de Ariño, Monografías Arqueológicas, Zaragoza. 59-62.

González-Sampériz, P. and Sopena, M.C., 2002 'Recent Holocene palaeoenvironmental evolution in the Central Ebro Basin (N.E. Spain)', Quaternary International 93, 177-90.

González-Sampériz, P., Valero-Garcés, B., García-Ruiz, J.M. and Martí, C., 2003a 'Lateglacial and Holocene vegetational change in the Pyrenees and the Central Ebro Valley (NE Spain)', in M.B. Ruiz Zapata, M. Dorado Valiño, A.
Valdeolmillos Rodríguez, M.J. Gil García, T. Bardají Azcárate, I. De Bustamante Gutiérrez and I. Martínez Mendizábal (eds), Quaternary Climatic Changes and environmental crises in the Mediterranean Region, Alcalá de Henares, Madrid. 181-90.

González-Sampériz, P., Montes, L. and Utrilla, P., 2003b 'Pollen in hyaena coprolites from Gabasa Cave (northern Spain)', Review of Palaeobotany and Palynology 126, 715.

González-Sampériz, P., Valero-Garcés, B., Carrión, J., PeñaMoné, JL., García-Ruiz, J.M. and Martí-Bono, C., 2005 'Glacial and Late glacial vegetation in Northeastern Spain, new data and a review', Quaternary International 141, 420.

González-Sampériz, P., Valero-Garcés, B. L., Moreno, A., Jalut, G., García-Ruiz, J.M., Martí-Bono, C., Delgado-Huertas, A., Navas, A., Otto, T. and Dedoubat J.J., 2006 'Climate variability in the Spanish Pyrenees for the last 30,000 yr, El Portalet peatbog sequence', Quaternary Research 66. 38-52.

González-Sampériz, P; Valero-Garcés, B. L; Moreno, A; Morellón, M; Navas, A; Machín, J. and Delgado-Huertas, A., 2008 'Vegetation changes and hydrological fluctuations in the Central Ebro Basin (NE Spain) since the Lateglacial, saline lake records', Palaeogeography, Palaeoclimatology, Palaeoecology 259, 157-81.

Guerrero, V.M. and Gornés, S., 2000 Colonización humana en ambientes insulares. Interacción con el medio y adaptación cultural, Universidad de Baleares, Palma de Mallorca.

Guilbaud, M., Sala, R., Cabañas, A.M. and Carbonell, E., 1993 'L'occupation acheuléene du Montgrí: Caus del Duc de Torroella et d'Ulla (Catalogne, Espagne)', in Instituto Tecnológico Geominero de España (ed), El Cuaternario en España y Portugal 1, Instituto Tecnológico Geominero de España, Madrid. 399-408.

Havinga, A.J., 1984 'A 20-year experimental investigation into the differential corrosion susceptibility of pollen and spores in various soil types', Pollen et Spores 26, 541-58.

Heim C., Nowaczyk N., Negendank J., Leroy S. and Ben-Avraham Z., 1997 'Middle-East desertification: evidence from the Dead Sea', Naturwissenschaften 84(9), 398-401.

Hernández, F. and Galán, E., 1992 'La necrópolis de El Mercadillo (Botija, Cáceres)’, Extremadura Arqueológica 6, 1-156.

Hernández, F., Rodríguez, M.D. and Sánchez, M.A., 1989 Excavaciones en el Castro de Villasviejas de Tamuja (Botija, Cáceres). Junta de Extremadura, Mérida. 
Holloway, R.G., 1989 'Experimental mechanical pollen degradation and its application to Quaternary age deposits', Texas Journal of Science 41, 131-45.

Horowitz, A., 1992 Palynology of Arid Lands. Elsevier, Amsterdam.

Horrocks, M., Salter, J., Braggins, J., Nichol, S., Moorhouse, R. and Elliott, G., 2008 'Plant microfossil analysis of coprolites of the critically endangered kakapo (Strigops habroptilus) parrot from New Zealand', Review of Palaeobotany and Palynology, 229-45.

Hunt, C.O. and Rushworth, G., 2005 'Pollen taphonomy and airfall sedimentation in a tropical cave, the West Mouth of The Great Cave of Niah in Sarawak, Malaysian Borneo', Journal of Archaeological Science 32, 465-73.

Hunt, C.O., Rushworth, G., Gilbertson, D.D. and Mattingly, D.J., 2001 'Romano-Libyan dryland animal husbandry and landscape, pollen and palynofacies analyses of coprolites from a farm in the Wadi el-Amud, Tripolitania', Journal of Archaeological Science 28, 351-63.

Hurtado, V., 2004 'El asentamiento fortificado de San Blás (Cheles, Badajoz). III milenio AC', Trabajos de Prehistoria 61, 141-55.

Hurtado, V., 2007 'Interpretación sobre la dinámica cultural en la Cuenca Media del Guadiana (IV-III milenios A.N.E.)', Extremadura Arqueológica 5, 53-80.

Iglesias-Gil, J.M., 1997 Arqueología en Lulobriga (Retortillo, Campoo de Enmedio, Cantabria). Servicio de Publicaciones de la Universidad de Cantabria, Santander.

Iriarte, M.J., 1992 'El entorno vegetal en las Bárdenas Reales (Navarra) durante la prehistoria reciente', Historia 20, 359-67.

Iriarte, M.J., 1994 El paisaje vegetal de la Prehistoria reciente en el alto valle del Ebro y sus estribaciones atlánticas. PhD Thesis, Universidad del País Vasco, Vitoria.

Jones, J., Tinsley, H. and Brunning, R., 2007 'Methodologies for assessment of the state of preservation of pollen and plant macrofossil remains in waterlogged deposits', Environmental Archaeology 12, 71-86.

Jordán, J.F., 1992 'Prospección arqueológica en la Comarca de Hellín-Tobarra. Metodología, resultados y bibliografía', Al-Basit 31, 183-227.

Julià, R., Negendank, J.F.W., Seret, G., Brauer, A., Burjachs, F., Endres, Ch., Giralt, S. and Roca, J.R., 1994a Origin and Evolution of Desertification in the Mediterranean Environment in Spain. Final Report of the Project EV5VCT91-0037, Brussels.
Julià, R., Negendank, J.F.W., Seret, G.; Brauer, A., Burjachs, F., Endres, C., Giralt, S., Parés and J.M., Roca, J.M., 1994b 'Holocene climatic change and desertification in Western Mediterranean. Schriften der Alfred Wegener Stiftung', Terra Nostra 1/94, 81-84.

Julià, R., Giralt, S., Burjachs, F. and Roca, J.R., 2000 ‘Inferring water level changes in the Gallocanta closed-basin from ultra highresolution lake sediment stratigraphy', Terra Nostra 7, 40-44.

Lartigot, A.-S., 2007 Taphonomie pollinique en grotte de sédiments détritiques et de spéléothèmes, potentiels et limites pour la reconstitution de l'environnement végétal de l'homme préhistorique sur le pourtour nord-méditerranéen. Application aux sites de la Caune de l'Arago (Tautavel, Pyrénées Orientales, France), de la Baume Bonne (Quison, Alpes-de-Haute-Provence, France), de la Grotte de Lazaret (Nice, Alpes-Maritimes, France) et de la Grotte de la Basura (Toirano, Ligurie, Italie). PhD Thesis, Museum National d'Histoire Naturelle, Paris.

Lasheras, J.A. and de las Heras, C., 1997 'La cueva de Altamira: historia de un monumento', in CSIC (ed), La cristalización del pasado: génesis y desarrollo del marco institucional dela arqueología en España, Universidad de Málaga, Servicio de Publicaciones. 359-68.

Leroi-Gourhan, A., 1980 'Análisis polínico de la cueva de El Pendo', in J. González Echegaray (ed), El yacimiento de la cueva de El Pendo (excavaciones 1953-1957), Biblioteca Praehistorica Hispana, CSIC, Madrid. 265-66.

Leroy S.A.G., 1990 Paléoclimats plio-pléistocènes en Catalogne et Languedoc d'après la palynologie de formations lacustres. Vols 1 and 2. PhD Thesis, UCL.

Leroy, S.A.G., 1997 'Climatic and non-climatic lake-level changes inferred from a Plio-Pleistocene lacustrine complex of Catalonia (Spain), palynology of the Tres Pins sequences', Journal of Paleolimnology 17, 347-67.

Leroy S.A.G., 2008 'Vegetation cycles in a disturbed sequence around the Cobb-Mountain subchron in Catalonia', Journal of Palaeolimnology 40, 851-68.

Leroy, S.A.G. and Simms, M.J., 2006 'Iron age to medieval entomogamous vegetation and Rhinolophus hipposideros roost in South-Eastern Wales (UK)', Palaeogeography, Palaeoclimatology, Palaeoecology 237, 4-18.

López-García, P., 1981 'Análisis polínico del yacimiento de Los Azules (Cangas de Onís, Oviedo)', Botanica Macaronesica $9,243-48$.

López-García, P., 1983 'Análisis polínicos de cinco fondos de cabaña del km. 7 derecha de la carretera de San Martín de La Vega (T.M. Getafe, Madrid)', Estudios de Prehistoria y Arqueología Madrileñas 2, 266-70. 
López-García, P., 1986 'Estudio palinológico del Holoceno español a través del análisis de yacimientos arqueológicos', Trabajos de Prehistoria 43, 143-58.

López-García, P., 1988 'Estudio polínico de seis yacimientos del sureste español, Cueva de Ambrosio, Cueva del Calor, Abrigo del Milano, Almizaraque, El Prado, Abrigo de los Carboneros', Trabajos de Prehistoria 45, 335-45.

López-García, P., 1991 El cambio cultural del IV al II milenios a. C. en la Comarca Noroeste de Murcia. Volumen I. Consejo Superior de Investigaciones Científicas. Madrid.

López-García, P., 1997 El Paisaje Vegetal de la Comunidad de Madrid durante el Holoceno Final. Serie Arqueología, Paleontología y Etnografía, Monográfico 5. Consejería de Educación y Cultura de la Comunidad de Madrid, Madrid.

López-García, P. and López-Sáez, J.A., 1994a 'El paisaje andaluz en la Prehistoria, datos paleopalinológicos', Revista Española de Micropaleontología 26, 49-59.

López-García, P. and López-Sáez, J.A., 1994b 'Comparison of peats and archaeological samples in the Andalusian region, Spain', in O.K. Davis (ed), Aspects of Archaeological Palynology, Methodology and Applications, American Association of Stratigraphic Palynologists Contributions Series 29, Houston, Texas. 127-39.

López-García, P. and López-Sáez, J.A., 1997 Contribución al conocimiento de la historia de la vegetación de la provincia de Sevilla, análisis polínico del yacimiento arqueológico de "Los Molares"، Mediterranea, Serie de Estudios Biológicos 16, 19-22.

López-Sáez, J.A. and López-Merino, L., 2007 'Paleoambiente y paleoeconomía en la transición Epipaleolítico-Neolítico en la Alta Extremadura, análisis palinológicos en el yacimiento de la Canaleja (Romangordo)', in E.Cerrillo Cuenca, and A. González Cordero (eds), Cuevas para la eternidad, sepulcros prehistóricos de la provincia de Cáceres. Ataecina 3, Colección de Estudios Históricos de la Lusitania, Badajoz. 57.

López-Sáez, J.A., López-García, P. and Burjachs, F., 2003 ‘Arqueopalinología, síntesis crítica', Polen 12, 5-35.

Lozano, J.M., Ramos, J., García, E., Herrero, N., Pérez, M., Castañeda, V. and Lazarich, G., 1997 'El Retamar (Puerto Real, Cádiz), un asentamiento neolítico especializado en la pesca y el marisqueo', in P. Bueno and R. Balbín (eds), II Congreso de Arqueología Peninsular, Zamora, Fundación Rei Afonso Henriques. 49-58.

Løvlie, R. and Leroy, S., 1995 'Magnetostratigraphy of Lower Pleistocene Banyoles palaeolake carbonate sediments from Catalonia, NE Spain: evidence for relocation of the Cobb Mountain sub-chron', Quaternary Science Reviews
$14,473-85$.

Luly, J.G., 1997 'Modern pollen dynamics and surficial sedimentary processes at Lake Tyrrel, semiarid northwestern Victoria, Australia', Review of Palaeobotany and Palynology 97, 301-18.

Macphail, R.I., Cruise, G.M., Allen, M.J., Linderholm, J. and Reynolds, P., 2004 'Archaeological soil and pollen analysis of experimental floor deposits; with special reference to Butser Ancient Farm, Hampshire, UK', Journal of Archaeological Science 31, 175-191.

Mariscal, B., 1991a 'Fluctuación climática y variación de la vegetación durante el período Subboreal. Análisis polínico del Cerro de las Cuartillas, Mojácar (Almería)', Boletín Geológico y Minero 102, 556-61.

Mariscal, B., 1991b 'Características ambientales durante el Holoceno en las Pilas de Mojácar, Almería. Análisis polínico en la Cuenca del Río Aguas', Boletín Geológico y Minero 102, 394-99.

Mariscal, B., 1991c 'Características climáticas y ambientales durante el Holoceno en Almizaraque. Análisis polínico de los sedimentos fluviales de Villaricos (Almería)', Boletín Geológico y Minero 102, 726-34.

Mariscal, B., 1992 'Variación de la vegetación durante el Subboreal. Análisis polínico en Cabezo de Brujas, Almizaraque (Almería)', Revista Española de Micropaleontología 23, 141-49.

Mariscal, B., 1993 'Analisis polinico del yacimiento de la edad del bronce de el acequion. Albacete', Cuadernos de Prehistoria y Arqueología 20, 59-72.

Mariscal, B., 1994 'Variación de la vegetación de Soria según el registro polínico del yacimiento de Fuentesauco (2700-2200 B.P.)', in Actas 11 Congreso Nacional del Medio Ambiente, Ministerio de Medio Ambiente, Madrid. 377-92.

Mariscal, B., 1996 'Evolución de la vegetación desde el Calcolítico a la Primera Edad del Hierro en la Cuenca Media del Tajo', in B. Ruiz Zapata (ed), Estudios Palinológicos, Universidad de Alcalá de Henares, Alcalá de Henares. 83-86.

Martí Oliver, B., 1983 El naiximent de l'agricultura en el País Valencia. Del Neolític a l'Etat del Bronze. Universitat de Valencia, Cultura Universitaria Popular 1, Valencia.

Martí Oliver, C., González-Sampériz, P., Valero-Garcés, B. and García-Ruiz, J.M., 2002 'El depósito glaciolacustre de Linas de Broto (Pirineo Aragonés), y sus implicaciones paleoambientales', in A. Pérez-González, J. Vegas and M. Machado (eds), Aportaciones a la Geomorfología de España en el inicio del Tercer Milenio, Instituto Geológico y Minero de España, Madrid. 77-83. 
Martín-Arroyo, T., Ruiz Zapata, B., Valdeolmillos, A. and Benito, G., 1996a 'Reconstrucción de la vegetación durante el cuaternario reciente en El Puente del Arzobispo (Toledo, España), primeros datos polínicos', in B. Ruíz Zapata (ed), Estudios Palinológicos, Universidad de Alcalá de Henares. 91-94.

Martín-Arroyo, T., Ruiz Zapata, B. and Pérez Gónzalez, A., 1996b 'Paleoambiente en el valle del río Tajo durante el Pleistoceno superior, primeros datos polínicos', in P. Ramil Rego, C. Fernández Rodríguez and M. Rodríguez Guitián, (eds), Biogeografía Pleistocena-Holocena de la Península Ibérica, Xunta de Galicia, Santiago de Compostela. 73-86.

Martínez-Andreu, M. and Sánchez, J.L., 2006 'Cueva de la Higuera (Isla Plana, Cartagena)', in M. Lechuga (ed), Abstracts of the XVII Jornadas de Patrimonio Histórico en la región de Murcia, Murcia. 45-46.

Martínez-Sánchez, C., 1992 'Cueva Antón (Mula)', Abstracts of the III Jornadas de Arqueología Regional Murcia, University of Murcia. 7-8.

Martín-Puertas,C., Valero-Garcés, B., Mata, P., González-Sampériz, P., Bao, R; Moreno, A. and Stefanova, V., 2008 'Arid and humid phases in Southern Spain during the last 4000 years: The Zoñar Lake record, Córdoba', The Holocene 18(6), 907-21.

Martínez-Tudela, A., 1986 Los travertinos del Río Matarraña, Beceite, Teruel. Estudio paleobotánico, $\mathrm{PhD}$ Thesis, Universidad de Valencia, Valencia.

Mas, M., Jordá, J. and Ripoll, S., 1998 'Las cuevas de levante (Benalup, Cádiz), un yacimiento al aire libre del Paleolítico Superior en Andalucía suroccidental', Trabajos de Prehistoria 55, 21-33.

McGarry, S. and Caseldine, C.J., 2004 'Speleopalynology, a neglected tool in British Quaternary studies', Quaternary Science Reviews 23, 2389-404.

Menendez-Amor, J. and Florschütz, F., 1961 'Resultados del análisis polínico de una serie de turba recogida en la Ereta del Pedregal (Navarrés, Valencia)', Archivo de Prehistoria Levantina 9, 97-99.

Montes, L., 2005 'Abrigos de Legunova y Valcervera en Biel : campaña de 2004', Salduie. Estudios de Prehistoria y Arqueología 5, 255-68.

Montes, L. and Domingo, R., 2001 'Epipaleolítico y Neolítico en las Sierras Exteriores de Aragón. Prospecciones, sondeos y excavaciones 2001', Salduie. Estudios de Prehistoria y Arqueología 2, 323-36.
Montes, R., 1987 'Informe preliminar de las excavaciones realizadas en la Cueva de Los Aviones, Cartagena', Excavaciones y Prospecciones Arqueológicas 1, 3-4.

Montón, F.J., 1998 'Un poblado de la Edad del Hierro en Huesca’, Revista de Arqueología 208, 60.

Montoya, P., Alberdi, M.T., Barbadillo, L.J., van der Made, J., Morales, J., Murelaga, X., Peñalver, E., Robles, F., Ruiz Bustos, A., Sánchez, A., Sanchiz, B., Soria, D. and Szyndlarg, Z., 2001 'Une faune très diversifiée du Pléistocène inférieur de la Sierra de Quibas (province de Murcia, Espagne)', Earth and Planetary Sciences 332, 387-93.

Moore, P.D., 1986 'Hydrological changes in mires', in B.E. Berglund (ed), Handbook of Holocene Palaeoecology and Palaeohydrology, John Wiley, Chichester. 91-107.

Moore, P.D., Webb, J.A. and Collinson, M.E., 1991 Pollen Analysis. 2nd Edn, Oxford, Blackwell.

Morellón, M., Valero-Garcés, B., Moreno, A., González-Sampériz, P., Mata, P., Romero, O., Maestro, M. and Navas, A., 2008 'Holocene palaeohydrology and climate variability in northeastern Spain: The sedimentary record of Lake Estanya (Pre-Pyrenean Range)', Quaternary Internacional 181, 15-31.

Moreno, A., Valero-Garcés, B.L., González-Sampériz, P., Navas A., Machín, J. and Delgado-Huertas, A., 2004 'El registro paleoambiental y paleoclimático de las saladas de La Playa y La Salineta (zona central de la Depresión del Ebro)', Geotemas 6, 137-40.

Moreno, A., Valero-Garcés, B., González-Sampériz, P. and Rico, M.T. 2008 'Flood response to rainfall variability during the last 2000 years inferred from the Taravilla Lake record (Central Iberian Range, Spain)', Journal of Palaeolimnology 40, 943-961. DOI 10.1007/s10933-008-9209-3.

Muñoz, K., 2000 'The Tagus Middle Basin (Iberian Peninsula) from the Neolithic to the Iron Age (V-I millenium cal. BC), the long way to social complexity', Oxford Journal of Archaeology 19, 241-72.

Muñoz-Sobrino, C., Ramil-Rego, P. and Gómez-Orellana, L., 2004 'Vegetation of the Lago de Sanabria area (NW Iberia) since the end of the Pleistocene: a palaeoecological reconstruction on the basis of two new pollen sequences', Vegetation History and Archaeobotany 13, 1-22.

Nájera, T. and Molina, F., 1977 'Edad del Bronce en La Mancha, excavaciones en las Motillas del Azuer y Los Palacios (campaña 1974)', in Cuadernos de Prehistoria de la Universidad de Granada 2. 251-300. 
Nakagawa, T., Brugiapaglia, E., Digerfeldt, G., Reille, M., Baulieu, J.L. de, and Yasuda, Y., 1998 'Dense-media separation as a more efficient pollen extraction method for use with organic sediment/deposit samples: comparison with the conventional method', Boreas 27, 15-24.

Navarro, C., Carrión, J.S., Navarro, J., Munuera, M. and Prieto, A.R., 2000 'An experimental approach to the palynology of cave deposits', Journal of Quaternary Science 15, 60319.

Navarro, C., Carrión, J.S., Munuera, M. and Prieto, A.R., 2001 'A palynological study of karstic cave sediments on the basis of their potential for palaeoecological reconstruction', Review of Palaeobotany and Palynology 117, 24565 .

Navarro, C., Carrión, J.S., Prieto, A.R. and Munuera, M., 2002 'Modern cave pollen and its application to describe the palaeorecords in an arid environment', Complutum 13, 718.

Olària, C., Gusi, F., Carbonell, E., Ollé, A., Vallverdú. J., Allué, E., Bennàsar, L., Bischoff, J.L., Burjachs, F., Cáceres, I., Expósito, I., López-Polín, L., Saladié, P. and Vergès, J.M., 2007 'Noves intervencions al jaciment pleistocènic de la Cova de Dalt del Tossal de la Font (Vilafamés, Castelló)', Quaderns de Prehistòria i Arqueologia de Castelló 24, 926.

Oms, O., Parés, J.M., Martínez-Navarro, B., Agustí, J., Toro, I., Martínez-Fernández, G. and Turf, A., 2000 'Early human occupation of western Europe, Paleomagnetic dates for two paleolithic sites in Spain', Proceedings of the National Academy of Sciences 97, 10666-670.

Ortega, M., Velasco, J., Millán, A. and Guerrero, C., 2004 ‘An ecological integrity index for littoral wetlands in agricultural catchments of semiarid Mediterranean regions', Environmental Management 33, 412-30.

Palmqvist, P., Gröcke, D.R., Arribas, A. and Fariña, R.A., 2003 'Paleoecological reconstruction of a lower Pleistocene large mammal community using biogeochemical $\left(\delta^{13} \mathrm{C}\right.$, $\left.\delta^{15} \mathrm{~N}, \delta^{18} \mathrm{O}, \mathrm{Sr}, \mathrm{Zn}\right)$ and ecomorphological approaches', $P a$ leobiology 29, 205-29.

Pantaleón-Cano, J., Yll, E.I., Pérez-Obiol, R. and Roure, J.M., 2003 'Palynological evidence for vegetational history in semi-arid areas of the western Mediterranean (Almería, Spain)', The Holocene 13, 109-19.

Peñalba, M.C., 1994 'The history of the Holocene vegetation in northern Spain from pollen analysis', Journal of Ecology 82, 815-32.
Peñalba, M.C., Arnold, M., Guiot, J., Duplessy, J.-C. and de Beaulieu, J.-L., 1997 'Termination of the last glaciation in the Iberian Peninsula inferred from the pollen sequence of Quintanar de la Sierra', Quaternary Research 48, 205-14.

Peregrina, N., 2003 Terrassa fa un milió d'anys. Ajuntament de Terrassa, Regidoria de Cultura, Museu de Terrassa. Terrassa, Barcelona.

Pérez-González, A., Dupré, M., Santonja, M., Mora, R., Sesé, C., Villa, P., Eisenmann, V. and Soto, E., 2000 'El yacimiento paleolítico de Cuesta de la Bajada (Teruel)', in III Congresso de Arqueología Peninsular, UTAD, Vila Real, Portugal. 169-88.

Pérez-Obiol, R., 1988 'Histoire Tardiglaciaire et Holocène de la végétation de la région volcanique d'Olot (N.E. Péninsule Ibérique)', Pollen et Spores 30, 189-202.

Pérez-Obiol, R. and Julià, R., 1994 'Climatic change on the Iberian Peninsula recorded in a 30,000-yr pollen record from Lake Banyoles', Quaternary Research 41, 91-98.

Pons, A. and Reille, M., 1988 'The Holocene and Upper Pleistocene pollen record from Padul (Granada, Spain), a new study', Palaeogeography, Palaeoclimatology, Palaeoecology 66, 243-63.

Pons, E., 1977 La Fonollera (1 y 2 Campañas de excavación 1975-1976), ensayo metodológico y analítico de excavación y estudio de los materiales hallados en el poblado del Bronce Final de la Fonollera (Torroella de Montgrí. Girona), Servicio Técnico de Investigaciónes Arqueólogicas de la Excma, Diputación Provincial, Girona.

Postigo, J.M., Burjachs, F., Gómez-Manzaneque, F. and Morla, C., 2007 'A palaeoecological interpretation of the lowermiddle Pleistocene Cal Guardiola site (Terrassa, Barcelona, NE Spain) from the comparative study of wood and pollen samples', Review of Palaeobotany and Palynology 146, 247-64.

Prieto, A.R. and Carrión, J.S., 1999 'Tafonomía polínica, sesgos abióticos y bióticos del registro polínico en cuevas' in Asociación Paleontológica Argentina Spec. Publ. 6. 59-64.

Pueyo, E.L., Garcés, M., Mauritsch, H.J., Lewis, C., Scholger, R., Sancho, C., Molina, R., Schnepp, E., Larrasoaña, J.C., Parés, J.M., Pocoví, A., Muñoz, A., Valero, B., Millán, H., Laplana, C., Oliva, B. and González-Sampériz, P., 2006 'Sampling, transportation and magnetic-free consolidation of extremely soft sediments for paleomagnetic purposes, a successful "recipe", in M. Calvo, M. Garcés, C. Gomes, J.C. Larrasoaña, E. Pueyo and J.J. Villalaín (eds), Paleomagnetismo en la Península Ibérica, Universidad de Burgos. 121-28. 
Ramallo, S.F., San Martín, P.A. and Ruiz, E., 2004 Informe sucinto de los resultados obtenidos en las excavaciones arqueológicas desarrolladas en el Teatro de Cartagena durante el año 1997. Memorias de Arqueología 12, 33154.

Ramil-Rego, P. and Gómez-Orellana, L., 1996 'Dinámica climática y biogeográfica del área litoral-sublitoral de Galicia durante el Pleistoceno Superior-Holoceno', in P. Ramil-Rego (ed), Biogeografía pleistocena-holocena de la Península Ibérica, Santiago de Compostela. 43-71.

Ramil-Rego, P., Muñoz-Sobrino, C., Rodríguez-Guitián, M. and Gómez-Orellana, L., 1998 'Differences in the vegetation of the North Iberian Peninsula during the last 16,000 years', Plant Ecology 138, 41-62.

Reed, J., 1998 'Diatom preservation in the recent sediment record of Spanish saline lakes, implications for palaeoclimate study', Journal of Paleolimnology 19, 129-37.

Reed, J.M., Stevenson, A.C. and Juggins, S., 2001 'A mulltiproxy record of Holocene climatic change in southwestern Spain, the Laguna de Melina, Cadiz', The Holocene 11, 707-19.

Riquelme, J.A., Rodríguez-Vidal, J., Carmona, R. and Martínez-Aguirre, A., 2004 'La Cueva del Grajo II (Priego de Córdoba), informe de la Intervención Arqueológica de Urgencia y primeras actuaciones realizadas en el yacimiento paleontológico pleistocénico', Antiquitas 16, 5-26.

Rodó, X., Giralt, S., Burjachs, F., Comín, F.A., Tenorio, R.F. and Julià, R., 2002 'High-resolution saline lake sediments as enhanced tools for relating proxy paleolake records to recent climatic data series', Sedimentary Geology 148, 203-20.

Rodrigo, M.A., Armengol, X., Oltra, R. and Colom, W., 2002 'Physical and chemical characterization of a protected wetland area in El Fondo d'Elx (Alicante, Spain)', Limnetica 21, 37-46.

Rodríguez-Ramírez, A., Rodríguez-Vidal, J., Cáceres, L., Clemente, L., Belluomini, G., Manfra, L., Improta, S. and De Andrés, J.R., 1996 'Recent coastal evolution of the Doñana National Park (S.Spain)', Quaternary Science Reviews 15, 803-09.

Rodríguez-Sánchez, J.A. (ed), 1998 Guía para conocer y visitar el Parque Natural de la Sierra de Baza, Asociación Proyecto Sierra de Baza, Baza, Granada.

Roure, J.M., Yll, E.I., Pantaleón, J. and Pérez-Obiol, R., 2000 'Characterization of the aridity processes on Mediterranean Europe. Protection and Management Guidelines', ARIDUSEUROMED UE Project, unpublished final report.
Ruiz-Zapata, M.B., Farias, P., Jiménez-Sánchez, M., Gil-García, M.J., Dorado, M. and Valdeolmillos, A., 2000 'Secuencia polínica de un depósito de la depresión de Comeya (Picos de Europa, Asturias), implicaciones paleoclimáticas', in S. Moreno (ed), Actas XIII Simposio de la APLE, Cartagena. 379-89.

Ruiz-Zapata, M.B., Gil García, M.J. and Dorado Valiño, M., 2002 'Clima y vegetación durante el Tardiglaciar y el holoceno en la Sierra de Neila (Sistema Ibérico noroccidental)', Cuaternario y Geomorfología 16, 1-4.

Sánchez-Goñi, M.F., 1991 Analyses polliniques des remplissages de grotte de Lezetxiki, Labeko et Urtiaga (Pays Basque espagnol). Leur place dans le cadre des sequences polliniques de la côte cantabrique et des Pyrénées occidentales. De la taphonomie pollinique à la reconstitution de l'environnement, Thèse, Museum National d'Histoire Naturelle, Institut de Paleontologie Humaine, Paris.

Sánchez-Goñi, M.F., 1994 'The identification of European upper palaeolithic interstadials from cave sequences. Aspects of archaeological palynology, methodology and applications' in O.K. Davis (ed), Aspects of Archaeological Palynology, Methodology and Applications, AASP Contribution Series, Vol. 29. 161-82.

Sanchís, A.K., 1992 Análisis polínico de la secuencia de Cova de Bolumini. MsD Thesis. University of Valencia.

Sancho, C., Muñoz, A., Peña, J.L. and González-Sampériz, P., 2007 'Evolución morfosedimentaria del sistema aluvial de Valsalada (Leciñena, Los Monegros) durante el Pleistoceno superior-Holoceno, aproximación paleoambiental', Geogaceta 41, 199-202.

Santos, L., 2004 'The late Holocene forest history and deforestation dynamics in the Queixa Sierra, Galicia, northwest Iberian Peninsula', Mountain Research and Development 24, 251-57.

Santos, L., Vidal Romaní, J.R. and Jalut, G., 2000 'History of vegetation during the Holocene in the Courel and Queixa Sierras, Galicia, northwest Iberian Peninsula', Journal of Quaternary Science 15, 621-32.

Scott, L., 1982 'Pollen analyses of Late Cenozoic deposits in the Trasvaal, South Africa, and their bearing on palaeoclimates', Palaeoecology of Africa 15, 101-7.

Scott, L., 1987 'Pollen analysis of hyena coprolites and sediments from Equus Cave, Taung, Southern Kalahari (South Africa)', Quaternary Research 28, 144-56.

Scott, L., 1995 'Palaeoenvironmental conditions in South Africa at the Pleistocene-Holocene transition', Quaternary Science Reviews 14, 937-47. 
Scott, L., 1999 'Palynological analysis of the Pretoria Saltpan (Tswaing Crater) sediments and vegetation history in the bushveld savanna biome, South Africa', in T.C. Partridge (ed), Tswaing - Investigations into the Origin, Age and Palaeoenvironments of the Pretoria Saltpan, Council for Geosciences, Pretoria. 143-66.

Scott, L. and Cooremans, B., 1992 'Pollen in recent Procavia (hyrax), Petromus (dassie rat) and bird dung in South Africa', Journal of Biogeography 19, 205-15.

Scott, L. and Nyakale, M., 2002 'Pollen indications of Holocene palaeoenvironments at Florisbad spring in the central Free State, South Africa', The Holocene 12, 497 503 .

Scott, L., Fernández Jalvo, Y., Carrión, J.S. and Brink, J., 2003 'Preservation and interpretation of pollen in hyaena coprolites, taphonomic observations from Spain and southern Africa', Palaeontologia Africana 39, 83-91.

Sebastián, A. and Zozaya, J., 1991 'Informe de la tercera campaña de excavación en El Abrigo de Angel, Ladrunán, Teruel', Arqueología Aragonesa 84, 53-54.

Sousa, A. and García-Murillo, P., 1999 'Historical evolution of the Abalario lagoon complexes (Doñana Natural Park, SW Spain)', Limnetica 16, 85-98.

Stevenson, A.C., 1985 'Studies in the vegetational history of SW Spain. II. Palynological investigations at Laguna de las Madres, Huelva', Journal of Biogeography 12, 243 68 .

Stevenson, A.C., n.d. 'El Abalario. A vegetation history', unpublished report.

Stevenson, A.C. and Moore, P.D., 1988 'Studies in the vegetational history of SW Spain. IV. Palynological investigations of a valley mire at El Acebrón, Huelva', Journal of Biogeography 15, 339-61.

Stevenson, A.C., Macklin, M.G., Benavente, J. A., Navarro, C., Pasmore, D. and Davis, B.A., 1991 'Cambios ambientales durante el Holoceno en el valle medio del Ebro, sus implicaciones arqueológicas', Cuaternario y Geomorfología 5, 149-64.

Suárez, A., 1989 'Abdera, una colonia fenicia en el sureste de la Península Ibérica', Madrider Mitteilungen 30, 135-51.

Suc, J.-P., 1980 Contribution à la connaissance du Pliocène et du Pléistocène Inférieur des régions méditerranéennes d'Europe occidentale par l'analyse palynologique des dépôts du Languedoc-Roussillon (sud de la France) et de la Catalogne (Nord-Est de l'Espagne). PhD Thesis, University of Montpellier.
Taylor, D.M., Pedley, H.M., Davies, P. and Wright, M.W., 1998 'Pollen and mollusc records for environmental change in central Spain during the mid- and late Holocene', The Holocene 8, 605-12.

Tipping, R., 1987 'The origins of corroded pollen grains at five early postglacial pollen sites in western Scotland', Review of Palaeobotany and Palynology 53, 151-61.

Turner, C. and Hannon, G.E., 1988 'Vegetational evidence for late Quaternary climatic changes in southwest Europe in relation to the influence of the North Atlantic Ocean', Philosophical Transactions of the Royal Society of London Series B 318, 451-85.

Utrilla, P. and Mazo, C. (eds), 2008 La Peña de las Forcas de Graus (Huesca). Un asentamiento reiterado desde el Magdaleniense Inferior al Neolitico Antiguo, Monografías Arqueológicas 43, Universidad de Zaragoza.

Utrilla, P., González-Sampériz, P., Ferrer, C. and Blasco, F., 2000 'La ocupación magdaleniense del valle del río Henar, los asentamientos de Cetina (Zaragoza) y Deza (Soria)', in Geoarqueología i Quaternari Litoral. Memorial-M.P. Fumanal, Universidad de Valencia, Valencia. 283-99.

Valero-Garcés, B., Delgado-Huertas, A., Navas, A., Machín, J., González-Sampériz, P. and Kelts, K., 2000a 'Quaternary paleohydrological evolution of a playa lake, Salada Mediana, central Ebro Basin, Spain', Sedimentology 47, 1135-156.

Valero-Garcés, B., González-Sampériz, P., Delgado-Huertas, A., Navas, A., Machín, J. and Kelts, K., 2000b 'Late Glacial and Late Holocene environmental vegetational change in Salada Mediana, central Ebro Basin, Spain', Quaternary Internacional 73, 29-46.

Valero-Garcés, B., González-Sampériz, P., Navas, A., Machín, J., Delgado-Huertas, A., Peña-Monné, J.L., Sancho, C., Stevenson, A. and Davis, B.. 2004 'Palaeohydrological fluctuations and steppe vegetation at the last Glacial Maximum in the Central Ebro valley (NE Spain)', Quaternary International 122, 43-55.

Valero-Garcés, B., González-Sampériz, P., Navas, A., Machín, J., Mata, P., Delgado, A., Bao, R., Moreno, A., Carrión, J.S., Schwalb, A. and González-Barrios, A., 2006a 'Human impact since Medieval times and recent ecological restoration in a Mediterranean Lake, The Laguna Zóñar, Spain', Journal of Paleolimnology 35, 441-65.

Valero-Garcés, B; González-Sampériz, P, Morellón, M; Rico M; Moreno A, Navas, A, Mata-Campo, P. and Rubio, J.C., $2006 \mathrm{~b}$ 'A new long ( $>250 \mathrm{ka}$ ?) lacustrine record from the Iberian Range (The Villarquemado wetland, Teruel), climate and tectonic implications for Late Quaternary evolution in NE Spain', Abstract Book of AMQUA Symposium (American Quaternary Association ), Bozeman (Montana, EEUU). 
Vallverdú, J., Courty, M.-A., Carbonell, E., Canals, A. and Burjachs, F., 2001 'Les sédiments d'Homo antecessor de Gran Dolina, (Sierra de Atapuerca, Burgos, Espagne). Interprétation micromorphologique des processus de formation et enregistrement paléoenvironnemental des sédiments', L'Anthropologie 105, 45-69.

van der Knaap, W.O. and van Leeuwen, J.F.N., 1994 'Holocene vegetation, human impact, and climatic change in the Serra da estrela, Portugal', Dissertationes Botanicae 234, 497-535.

van der Knaap, W.O. and van Leeuwen, J.F.N., 1995 'Holocene vegetation succession and degradation as responses to climatic change and human activity in the Serra de Estrela, Portugal', Review of Palaeobotany and Palynology 89, 153-211.

van Geel, B., Hallewas, D.P. and Pals, J.P., 1983 'A Late Holocene deposit under the Westfriese Zeedijk near Enkhuizen (Prov. of Noord-Holland, The Netherlands), Palaeoecological and archaeological aspects', Review of Palaeobotany and Palynology 38, 269-335.

van Geel, B., Buurman, J., Brinkkemper, O., Schelvis, J., Aptroot, A., van Reenen, G. and Hakbijl, T., 2003 'Environmental reconstruction of a Roman Period settlement site in Uitgeest (The Netherlands), with special reference to coprophilous fungi', Journal of Archaeological Science 30, 873-83.

van Leeuwaarden, J. and Janssen, C.R., 1985 'A preliminary palynological study of peat deposits near an Oppidum in the Lower Tagus Valley, Portugal', Actas Cuaternario Ibérico 2, 225-36.

van Mourik, J.M., 1985 Pollen Profiles of Slope Deposits in the Galician Area (NW Spain). Nederlandse Geografische Studies. Amsterdam.

Vaquero, M., 2001 'L'Abric Agut (Capellades, Anoia)', in M. Genera i Monells (ed), Actes de les Jornades d'Arqueologia i paleontología 2001, La Garriga, Comarques de Barcelona. 67-74.

Vaquero, M., 2006 'El tránsito Paleolítico Medio/Superior en la Península Ibérica y la frontera del Ebro', Pyrenae 37, 107-129.

Vega-Toscano, L.G., 1993 'Excavaciones en el Abrigo del Molino del Vadico (Yeste), el final del paleolítico y los inicios del neolítico en la Sierra Alta del Segura', in J. Blánquez (ed), Arqueología en Albacete, Jornadas de arqueología albacetense en la Universidad Autónoma de Madrid. 19-34.

Vega-Toscano, L.G., Hoyos, M., Ruiz-Bustos, A. and Laville, H., 1988 'La séquence de la grotte de la Carihuela (Píñar, Grenade): chronostratigraphie et paléoécologie du Pléistocène Supérieur au Sud de la Péninsule Ibérique', in M. Otter (ed), L'Homme de Néanderthal, Vol. 2. L'Environnement, Université de Liège. 169-80.
Vera, J.A. (ed), 2004 Geología de España. Instituto Geológico y Minero de España, Madrid.

Vermoere, M., Degryse, P., Vanhecke, L., Muchez, Ph., Paulissen, E., Smets, E. and Waelkens, M., 1999 'Pollen analysis of two travertine sections in Basköy (southwestern Turkey), implications for environmental conditions during the early Holocene', Review of Palaeobotany and Palynology 105, 93-110.

Villalta, J.F. and Vicente, J., 1972 'Una flora del Cuaternario antiguo en las cercanías de Crespià', Acta Geologica Hispánica 7, 120-28.

Villaverde, V., 2001 'Cova de les Cendres (Teulada-Moraira, Alacant)', in V. Villaverde (ed), De Neandertales a Cromañones. El inicio del doblamiento humano en las tierras valencianas, Universidad de Valencia, Valencia. 411-14.

Viñals, M.J., Belluomini,G., Fumanal, M.P., Dupré, M., Usera, J., Mestres, J. and Manfra, L., 1993 'Rasgos paleoambientales en la Bahía de Xàbia (Alicante)’, in M.P. Fumanal and J. Bernabeu (eds), Estudios sobre Cuaternario. 107-14.

Vives, E., 1982 ‘Antropología, La Cova de Les Calaveres’, Instituto de Prehistoria de Valencia 75, 45-47.

Weinstein-Evron, M., 1987 'Palynology of Pleistocene travertines from the Arava Valley, Israel', Quaternary Research 27, 82-88.

Williams-Dean, G., 1978 Ethnobotany and cultural ecology of prehistoric man in southwest Texas. PhD Thesis, Texas A. and M. University, College Station, Texas.

Yáñez, C., 2005 Evolución ambiental de las marismas del Guadalquivir durante el Holoceno reciente, estudios paleopalinológicos Mediterránea. PhD Thesis, University of Sevilla.

Yáñez, C., Rodríguez, A. and Carrión, J.S., 2006 'Cambios en la vegetación de la franja litoral de las marismas de Doñana durante el Holoceno reciente', Anales de Biología 28, 85-94.

Y1l, E.I., Roure, J.M., Pantaleón-Cano, J. and Pérez-Obiol, R., 1994 'Análisis polínico de una secuencia holocenica en Roquetas de Mar (Almeria)', in I. Mateu, M. Dupré, J. Güenes and M.E. Burgaz (eds), Trabajos de Palinología Básica y Aplicada, X Simposio de Palinología (APLE), Universitat de València. 189-98.

Yll, R., 1983 Dinámica de complexes arqueolòics del llevant peninsular entre 16.000 i 7.000 BP. MS Thesis, Autonomous University of Barcelona. 
Y1l, R., 1988 'Análisis de polen y palinograma', in C. Olaria (ed), Cova Fosca. Un asentamiento meso-neolítico de cazadores y pastores en la serranía del Alto Maestrazgo, Monografies de Prehistoria i Arqueologia castellonenques 3, Servicio de Arqueologia, Diputació de Castelló. 26578.

Yll, R., Carrión, J.S., Pantaleón, J., Dupré, M., La Roca, N., Roure, J.M. and Pérez-Obiol, R., 2003 'Palinología del Cuaternario reciente en la Laguna de Villena (Alicante, España)', Anales de Biología 25, 65-72.

Yll, R., Zazo, C., Goy, J.L., Pérez-Obiol, R., Pantaleón-Cano, J., Civis, J., Dabrio, C., González, A., Borja, F., Soler, V., Lario, J., Luque, L., Sierro, F., González-Hernández, F.M., Lèzine, A.M., Denèfle, M. and Roure, J.M., 2004 'Quaternary palaeoenvironmental changes in south Spain', in M.B. Ruiz-Zapata, A. Dorado, A. Valdeolmillos, M.J. Gil, T. Bardaji, I. de Bustamante and I. Martínez (eds), Quaternary Climatic Changes and Environmental Crises in the Mediterranean Region, Universidad de Alcalá de Henares (CD-Rom, 2002).

Yll R., Carrión J.S., Marra A.C. and Bonfiglio, L., 2006 'Pollen in late Pleistocene hyena coprolites from San Teodoro Cave (Sicily, Italy)', Palaeogeography, Palaeoclimatology, Palaeoecology 237, 32-39.

Y11, R., Expósito, I. and Burjachs, F., 2007a 'Informe de los análisis palinológicos del yacimiento paleolítico de Vallparadís (Terrassa, Barcelona)'. ArqueoLine, S.L., unpublished report.

Yll, R., Expósito, I. and Burjachs, F., 2007b 'Informe del análisis palinológico del yacimiento arqueológico de Cerro San Vicente (Salamanca)', unpublished report.

Y1l, R., Expósito, I. and Burjachs, F., 2007c 'Informe de l'anàlisi palinològica del jaciment arqueològic de Camp Vermell (Sant Julià de Lòria, Andorra)'. Patrimoni Cultural d'Andorra, Govern d'Andorra, unpublished report.

Y11, R., Expósito, I. and Burjachs, F., 2008a 'Informe de los análisis palinológicos de coprolitos de Crocuta procedentes del yacimiento paleolítico de Vallparadís (Terrassa,
Barcelona)'. ArqueoLine, S.L., unpublished report.

Yll, R., Expósito, I. and Burjachs, F., 2008b 'Informe de l'anàlisi palinològica del jaciment arqueològic del Turó de la Bateria (Girona)', unpublished report.

Y1l, R., Expósito, I. and Burjachs, F., 2008c 'Informe palinològic preliminar dels sondeigs MES1 i MES2 a Tornabous (Lleida)'. Museu Arqueològic Nacional de Catalunya, unpublished report.

Y11, R., Expósito, I. and Burjachs, F., 2008d 'Informe de l'anàlisi palinològica del jaciment arqueològic de Cova Matutano (Castelló)'. Universitat Jaume I (Castelló), unpublished report.

Zafra, N., Hornos, F. and Castro, M., 1999 'Una macro-aldea en el origen del modo de vida campesino, Marroquíes Bajos (Jaén) c. 2500-2000 cal. ANE', Trabajos de Prehistoria 56, 77-102.

Zilhao, J., 2001 'Middle Palaeolithic settlement patterns in Portugal', in D. Conard (ed), Settlement Dynamics of the Middle Palaeolithic and Middle Stone Age, Kerns Verlag, Tübingen. 597-608.

\section{Acknowledgements}

This article has been funded by the projects CGL20062956-BOS (Spanish Ministerio de Educación y Ciencia), and PaleoDiversitas (Fundación Séneca, Murcia). Reported failures are associated with a number of other projects, among which we select the following: EU: EV5V-CT91-0037, MEC: CG85117, PB86-0615, PB88-80, PB-88-90, PB90-79, PB-91-42, PB91-897, PB93-446, CLI97-445-C02, BOS2000-149, CGL2006-13327-C04-01/CLI, REN2003-09130-CO2-02, CARM-Fundación Séneca: PIB93-8, PI17-739-FS, CSIC: PIE200710I011, CAM-CCG07-HUM 1924, and several other minor funding actions by Junta de Andalucía, Gobierno de Aragón, Junta de Castilla-León, Instituto de Estudios Altoaragoneses, Instituto de Estudios Riojanos, IGME, and Instituto de Ciencias de la Tierra 'Jaume Almera' (CSIC). Mike Turner (Brunel University) kindly checked the English of an earlier version of this manuscript. 
